\title{
Endevaluierung des Förderprogramms austrian electronic network (AT:net)
}

Endbericht 
Diese Studie wurde im Auftrag des Bundesministeriums für Verkehr, Innovation und Technologie, Stabstelle Informations- und Kommunikationsinfrastruktur durchgeführt.

VERFASSER DES BERICHTS:

Sascha Ruhland (Projektleitung)

Laurenz Wolf

INTERNES REVIEW/BEGUTACHTUNG:

Sonja Sheikh

Die vorliegende Studie wurde nach allen Maßstäben der Sorgfalt erstellt.

Die KMU Forschung Austria übernimmt jedoch keine Haftung für Schäden oder Folgeschäden, die auf diese Studie oder auf mögliche fehlerhafte Angaben zurückgehen.

Dieses Werk ist urheberrechtlich geschützt. Jede Art von Nachdruck, Vervielfältigung, Verbreitung, Wiedergabe, Übersetzung oder Einspeicherung und Verwendung in Datenverarbeitungssystemen, und sei es auch nur auszugsweise, ist nur mit ausdrücklicher Zustimmung der KMU Forschung Austria gestattet.

Mitglied bei:

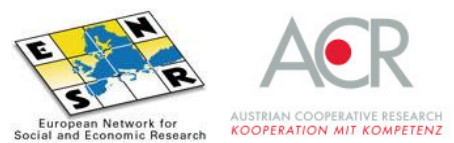




\section{Verzeichnis}

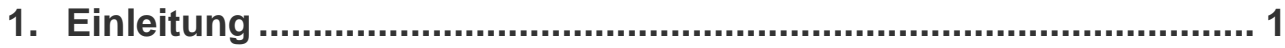

2. Konzept und Implementierung des Programms .............................. 3

2.1. Programmdesign und Interventionslogik ............................................. 3

2.2. Implementierung des Programms.................................................. 4

3. FFG-Beteiligung der in AT:net geförderten Organisationen...........10

4. Breitbandinfrastruktur und -nutzung in Österreich .........................19

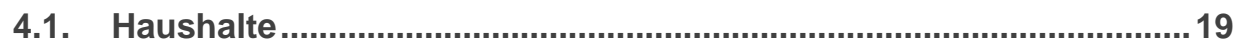

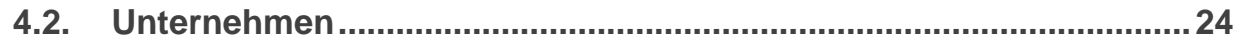

4.3. Internationale Position Österreichs ..............................................28

5. Wirkung der AT:net Förderung .....................................................33

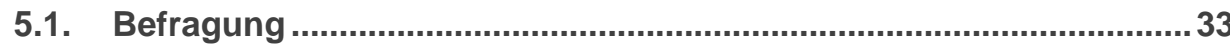

5.1.1. Befragte Organisationen .............................................. 33

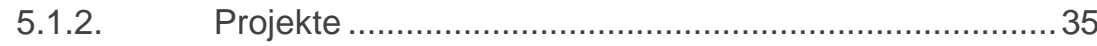

5.2. Projekt-/Programmimplementierung ..................................................41

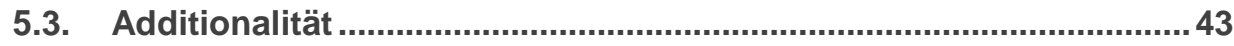

5.4. Projektergebnisse und -wirkungen ................................................... 45

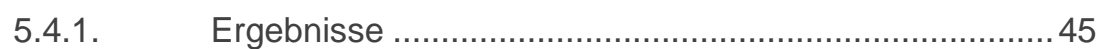

5.4.2. Wirkungen ........................................................ 51

6. Schlussfolgerungen und Handlungsempfehlungen......................60

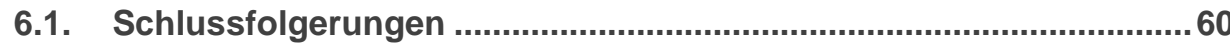

6.2. AT:net im internationalen Vergleich ...............................................63

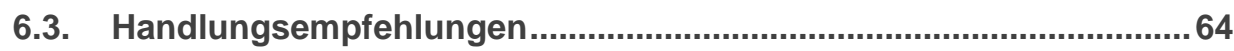




\section{Abbildungen}

Abbildung 1 In AT:net geförderte Organisationstypen nach Programmphase, in Prozent aller Fördernehmer

Abbildung 2 In AT:net geförderte Organisationen mit FFG Erstförderung nach Organisationstypen und Programmphasen, in Prozent aller Fördernehmer

Abbildung 3 Mittelausschöpfung des Programms nach Phasen und Ausschreibungen

Abbildung $4 \quad$ Eingereichte und genehmigte Projekte nach Phasen und Ausschreibungen....

Abbildung 5 Erfolgsquote der eingereichten Projekte nach Phasen und Ausschreibungen.

Abbildung 6 Beteiligungsprofil der in AT:net geförderten Organisationen in anderen FFG-Programmen auf Basis der Anzahl von geförderten Projekten 2001-2013, Programme mit 15 oder mehr Beteiligungen insgesamt

Abbildung 7 Beteiligungsprofil der in AT:net geförderten Organisationen in Clustern von Programmen auf Basis der Anzahl von geförderten Projekten 2001-2013

Abbildung 8 Beteiligung der in AT:net geförderten Organisationen in Clustern von Programmen, Anzahl von geförderten Projekten 2001-2013

Abbildung 9 Anteil der häufig geförderten Unternehmen an den Programmbeteiligungen aller in AT:net geförderten Organisationen, in \% aller Beteiligungen an Programmen mit insgesamt mehr als 10 Beteiligungen

Abbildung 10 Beteiligungsprofil der Unternehmen mit 10 oder mehr FFG-

Projekten, Anzahl der Beteiligungen an FFG-

Förderprogrammen

Abbildung 11 Relative Bedeutung von Clustern von Programmen für AT:net Fördernehmer, Vergleich Unternehmen mit 10 oder mehr Beteiligungen, 6-9 und 1-5 Beteiligungen, prozentualer Anteil der Beteiligungen an allen Beteiligungen der jeweiligen Gruppe.. 17

Abbildung 12 Absolute Bedeutung von Clustern von Programmen für AT:net Fördernehmer, Vergleich Unternehmen mit 10 oder mehr Beteiligungen, 6-9 und 1-5 Beteiligungen

Abbildung 13 Status - Österreichische Haushalte mit Interzugang und Breitbandverbindung in Prozent aller Haushalte bzw. in Prozent aller Haushalte mit Internetzugang, 2002-2013 ....

Abbildung 14 Entwicklungsdynamik - Österreichische Haushalte mit Interzugang und Breitbandverbindung, Veränderung zum Vorjahr in Prozentpunkten, 2003-2013

Abbildung 15 Status - Österreichische Haushalte mit Breitbandanbindung in Prozent aller Haushalte nach Haushaltsgröße, 2002-2013 
Abbildung 16 Status - Österreichische Haushalte mit Breitbandanbindung, Art der Anbindung in Prozent aller Haushalte nach Haushaltsgröße, 2013

Abbildung 17 Internetnutzung nach Altersgruppen, Entwicklung 2002-2013.....23

Abbildung 18 Status - Nutzungsverhalten österreichischer Internetnutzer nach Nutzungsart in Prozent aller Personen, 2013.

Abbildung 19 Status - Österreichische Unternehmen mit Interzugang und Breitbandanbindung in Prozent aller Unternehmen bzw. in Prozent aller Unternehmen mit Internetzugang, 2003-2013 .........25

Abbildung 20 Entwicklungsdynamik - Österreichische Unternehmen mit Interzugang und Breitbandverbindung, Veränderung zum Vorjahr in Prozentpunkten, 2004-2013....

Abbildung 21 Status - Österreichische Unternehmen mit eGovernment Aktivitäten nach Größenklassen, in Prozent aller Unternehmen mit eGovernment Aktivitäten, 2012

Abbildung 22 Status - Österreichische Unternehmen mit eCommerce Aktivitäten, Umsatzanteil eCommerce nach Größenklassen, in Prozent aller Unternehmen, Vergleich 2009 - 2012

Abbildung 23 Status - Österreichische Unternehmen mit eCommerce Aktivitäten, Anteil der Unternehmen mit Einkäufen über eCommerce nach Größenklassen, in Prozent aller Unternehmen, Vergleich 2009 - 2012

Abbildung 24 Status - Fixe Breitbandanbindung in der EU nach Technologie in Prozent aller Anschlüsse, 2013

Abbildung 25 Status - Next Generation Access (FTTH, FTTB, VDSL, Cable Docsis 3.0 und andere) in der EU in Prozent aller fixen Breibandanbindungen, 2013

Abbildung 26 Status - Fixe Breitanbindung nach Geschwindigkeit in der EU in Prozent aller Breitbandanbindungen, 2013.

Abbildung 27 Befragte Organisationen nach Organisationstyp...........................34

Abbildung 28 Befragte Unternehmen nach Größenklassen..................................35

Abbildung 29 Untersuchte Projekte: abgeschlossene vs. laufend .......................36

Abbildung 30 Untersuchte Projekte: Infrastrukturprojekte vs. andere ..................37

Abbildung 31 Primäres Ziel der untersuchten Projekte ......................................... 38

Abbildung 32 Erfahrung der befragten Organisationen mit anderen Fördermaßnahmen

Abbildung 33 Bewertung der Programmimplementierung durch die befragten Fördernehmer....

Abbildung 34 Additionalität der untersuchten Projekte....................................... 43

Abbildung 35 Input-Additionalität (Folgeinvestitionen) der abgeschlossenen Projekte

Abbildung 36 Zielerreichung der abgeschlossenen Projekte aus technischer und wirtschaftlicher Sicht ........................................................... 46

Abbildung 37 Verwertung der Projektergebnisse ...............................................48 
Abbildung 38 Projektergebnis als Teil des Angebotsportfolios der geförderten

Organisationen (abgeschlossene Projekte)

Abbildung 39 Innovationsgrad der Projektergebnisse bei Markteintritt ............... 50

Abbildung 40 Kosten-Nutzen-Relation der abgeschlossenen Projekte .............. 51

Abbildung 41 Zusätzliche erzielte Umsätze/Drittmittel, die direkt dem Projekt zugeordnet werden können ..................................................... 52

Abbildung 42 Arbeitsplatzwirkungen die direkt dem Projekt zugeordnet werden können, in Prozent aller befragten 53

Abbildung 43 Erschließung neuer Märkte in Folge des geförderten Projekts..... 54

Abbildung 44 Weitere Effekte als Folge des geförderten Projekts, in Prozent aller befragten Organisationen 55

Abbildung 45 Beitrag der abgeschlossenen geförderten Projekte zu

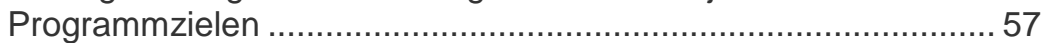

Abbildung 46 Beitrag der laufenden geförderten Projekte zu Programmzielen .. 59 


\section{Einleitung}

Der vorliegende Bericht beinhaltet die Ergebnisse der Endevaluierung des Förderprogramms austrian electronic network (AT:net). Das Ziel der von der KMU Forschung Austria in diesem Zusammenhang durchgeführten Evaluierung war es, einerseits die zentralen Fragestellungen hinsichtlich des Programmverlaufs über die gesamte Laufzeit 2007 - 2013 zu reflektieren und andererseits vor allem die Wirkung der Förderungen auf die geförderten Organisationen und die gesamtgesellschaftlichen Zieldimensionen zu analysieren. Die Ergebnisse der Untersuchungen wurden in entsprechende Empfehlungen in Hinblick auf eine etwaige Fortsetzung bzw. Neuauflage des Programms transformiert.

Diese Endevaluierung ist, aufbauend auf der Zwischenevaluierung des Programms, weniger auf die Konzeption und die Umsetzung des Programms fokussiert, sondern legt den Schwerpunkt vielmehr auf die Analyse der Zielerreichung sowie die zum jetzigen Zeitpunkt feststellbaren Wirkungen des Programms. Dementsprechend standen insbesondere folgende Leitfragen im Vordergrund der Endevaluierung:

- Ist das Förderungskonzept den Zielsetzungen angemessen bzw. waren die Maßnahmen geeignet, die strategischen Zielsetzungen des Programms zu erreichen?

- Konnten die operativen Zielsetzungen des Programms erreicht werden? Wie sind etwaige Abweichungen zu erklären?

- Welche intendierten und nicht-intendierten Wirkungen hat die Förderung bei den geförderten Organisationen erzielen können? Welche Rahmenbedingungen und andere Faktoren beeinflussen die Wirksamkeit?

- Welche Schlussfolgerungen bzw. Empfehlungen lassen sich in Hinblick auf eine etwaige Fortsetzung des Programms ableiten?

Der Bericht ist in 5 Hauptkapitel gegliedert. Kapitel 2 beinhaltet ein kurze Darstellung der Interventionslogik und des Programmdesigns sowie eine Analyse der Implementierung, d.h. geförderte Projekte/Organisationen, Mittelausschöpfung, Begleitmaßnahmen etc. Daran anschließend analysiert Kapitel 3 die Beteiligung der AT:net Fördernehmer an anderen FFG Programmen im Sinne einer Untersuchung der Forschungsintensität der Fördernehmer und der entsprechend relevanten Forschungsfelder und -themen. Kapitel 4 fasst zentrale Ergebnisse der Untersuchung der breitbandrelevanten Indikatoren zusammen, zu denen AT:net laut Programmdesign beitragen sollte. Die Ergebnisse der Befragung der Fördernehmer bezüglich der durchgeführten Projekte und erzielten Wirkungen auch über die Fördernehmer hinaus werden in Kapitel 5 analysiert. Im daran anschließenden Kapitel 6 werden basierend auf den vorangegangenen Analysen die entsprechen- 
den Schlussfolgerungen gezogen und in Handlungsempfehlungen bezüglich einer Weiterführung bzw. Neuauflage des Programms transformiert. 


\section{Konzept und Implementierung des Pro- gramms}

\subsection{Programmdesign und Interventionslogik}

Das Programm austrian electronic network adressiert vorrangig Klein- und Mittelunternehmen, welche IKT-Lösungen (d. h. Dienste und Anwendungen) „im öffentlichen Interesse unter Nutzung der Breitbandtechnologie einführen" - es wird somit die Markteinführung gefördert, prototypische Lösungen müssen schon vorhanden sein. AT:net versucht über die Anregung der Angebotsseite innovative und wirtschaftlich nachhaltige Geschäftsmodelle auf Basis von Breitbandtechnologie zu unterstützen und darüber den Markt zu stimulieren. Unter der Annahme, dass ein vorhandenes Angebot die Nachfrage bedingt bzw. anregt, soll diese Stimulierung nicht nur auf einer unternehmerischen Ebene (wie z. B. Marktzugang, Wettbewerbsfähigkeit der Unternehmen insgesamt), sondern auch auf einer gesellschaftlichen Makro-Ebene wirken, indem das technologische Niveau der Volkswirtschaft angehoben wird und eine Mehrheit der Bevölkerung an der so genannten Informationsgesellschaft teilhaben kann. Der Ausbau der Infrastruktur soll die Nachfrage einerseits ermöglichen (Möglichkeit der Nutzung von Breitband somit gegeben), andererseits besteht natürlich eine Wechselwirkung: die verstärkte Nachfrage nach Diensten und Anwendungen erhöht den Bedarf an Breitband-Infrastruktur.

Darüber hinaus ist das Programm AT:net explizit mit der Positionierung Österreichs in einigen der internationalen Vergleichsrankings verknüpft, die die Breitbandversorgung und -nutzung messen. Der Wirkungsmechanismus soll also Prozesse initiieren, die weit über den nationalen Kontext hinausgehen. Dazu war AT:net im Rahmen der zweiten Phase des Programms explizit mit dem Ziel der Schaffung eines europäischen Informationsraums bis 2020 verknüpft worden. Die vier zentralen Elemente der entsprechenden gesamteuropäischen Strategien beinhalten folgende Aspekte: schnellere Breitbanddienste, Verbesserung der Rahmenbedingungen für neue Dienste und Inhalte, Interoperabilität von Geräten und Diensten sowie Erhöhung der Sicherheit. Darüber hinaus soll AT:net einen zentralen Beitrag dazu leisten, Österreich in den internationalen Benchmarks (u. a. Network Readiness Index, E-Readiness Index etc.) unter den besten fünf Nationen zu platzieren. In diesem Zusammenhang sind auch jene operativen Ziele des Programms von Bedeutung, die sich auf die (bevölkerungsbezogene) Internetnutzung und die Versorgung mit Breitbandinternet beziehen: letzteres soll bis 2013 auf $70 \%$ steigen, ersteres auf $80 \%$ (siehe dazu die Analyse der entsprechenden Indikatoren in Kapitel 2).

Das Programm AT:net ist als Weiterführung bzw. Weiterentwicklung der bis 2006 existierenden so genannten „Breitbandinitiative“ zu betrachten. Während sich letzt- 
genanntes Programm ausschließlich dem Ausbau der vorhandenen Infrastruktur widmete, folgt AT:net dem Ansatz, diese Infrastruktur einer vielfältigen, innovationsorientierten und wettbewerbsfähigen Nutzung zuzuführen, wobei auch makroökonomische Ziele wie etwa die Beseitigung des so genannten „digital divide“ oder allgemeine konjunkturelle Wirkungen miteinbezogen wurden. In Phase 2 des Programms wurden mit den Mitteln des Konjunkturbelebungspakets auch Infrastrukturprojekte gefördert, in der dritten Phase wurden diese allerdings wieder aus der Interventionslogik genommen. Die Projekte konnten sowohl von einzelnen Organisationen als auch in Form von Kooperationsprojekten eingereicht werden.

Die Sonderrichtlinie für die aktuell dritte Programmphase des Programms verdeutlicht den Hintergrund sowie die Interventionslogik und die entsprechenden Zieldimensionen. Demnach basiert AT:net, wie oben bereits erwähnt, auf der Annahme, dass die hohen erwarteten Beiträge der Informations- und Kommunikationstechnologien zur Wertschöpfung in erster Linie über eine gestärkte Nachfrage realisiert werden können, die wiederum durch „attraktive Inhalte und Dienste“ ausgelöst wird. Als operatives Ziel sollte bis 2013 eine flächendeckende Versorgung Österreichs mit Bandbreiten von $25 \mathrm{Mb} / \mathrm{s}$ oder mehr realisiert sein. Allerdings scheint zumindest im Sinne der Nutzung ein solches Ziel bislang noch außer Reichweite zu sein (siehe Kapitel 4.3), vor allem vor dem Hintergrund der noch deutlich ehrgeizigeren Ziele der Digitalen Agenda der Europäischen Kommission (flächendeckende Bandbreiten von $30 \mathrm{Mb} / \mathrm{s}$ bis 2020 , davon $50 \%$ mit $100 \mathrm{Mb} / \mathrm{s}$ oder mehr).

Des Weiteren bezieht sich dieses Dokument auf die Frage nach der digitalen Kluft, also der so genannten elnklusion, insbesondere mit Hinblick auf soziale und gesundheitsbezogene IKT-Angebote für die alternde Gesellschaft sowie Unsicherheit (subjektiv) und mangelnde Kenntnisse aufseiten der (potenziellen) Nutzer. Dazu gibt die Sonderrichtlinie das Ziel aus, bis 2013 80\% der Österreicherlnnen zwischen 16 und 74 Jahren als aktive InternetnutzerInnen verzeichnen zu können.

Inwieweit AT:net die gesteckten Ziele erreichen bzw. dazu beitragen konnte, wird in den Kapiteln 4 und 5 vor dem Hintergrund der entsprechenden Indikatoren bzw. Projektergebnisse diskutiert.

\subsection{Implementierung des Programms}

Insgesamt sind über die Laufzeit des Programms 230 Organisationen in 208 AT:net Projekten gefördert worden (4 Organisationen waren an Projekten beteiligt, aber nicht förderfähig, da sie ihren Sitz nicht in Österreich haben). Davon waren 21 Organisationen an mehr als einem Projekt beteiligt: 14 an 2 Projekten, 6 an 3 Projekten und eine Organisation an 4 Projekten. In der ersten Phase wurden in AT:net 114 Projekte gefördert, in der zweiten Phase 63 und in der dritten Phase 42. 
Abbildung 1 zeigt die geförderten Organisationen nach Typen, wobei offensichtlich wird, dass KMU den größten Anteil ausmachen, der sich über die Laufzeit hinweg zusätzlich noch erhöht hat. Im Gegenzug ist der Anteil von Hochschulen, Intermediären (z.B. Fachverbände, Cluster oder Netzwerkorganisationen) und sonstigen Organisationen (z.B. Verbände oder Gebietskörperschaften) auf null zurückgegangen.

\section{Abbildung 1 In AT:net geförderte Organisationstypen nach Programmphase, in Pro- zent aller Fördernehmer}

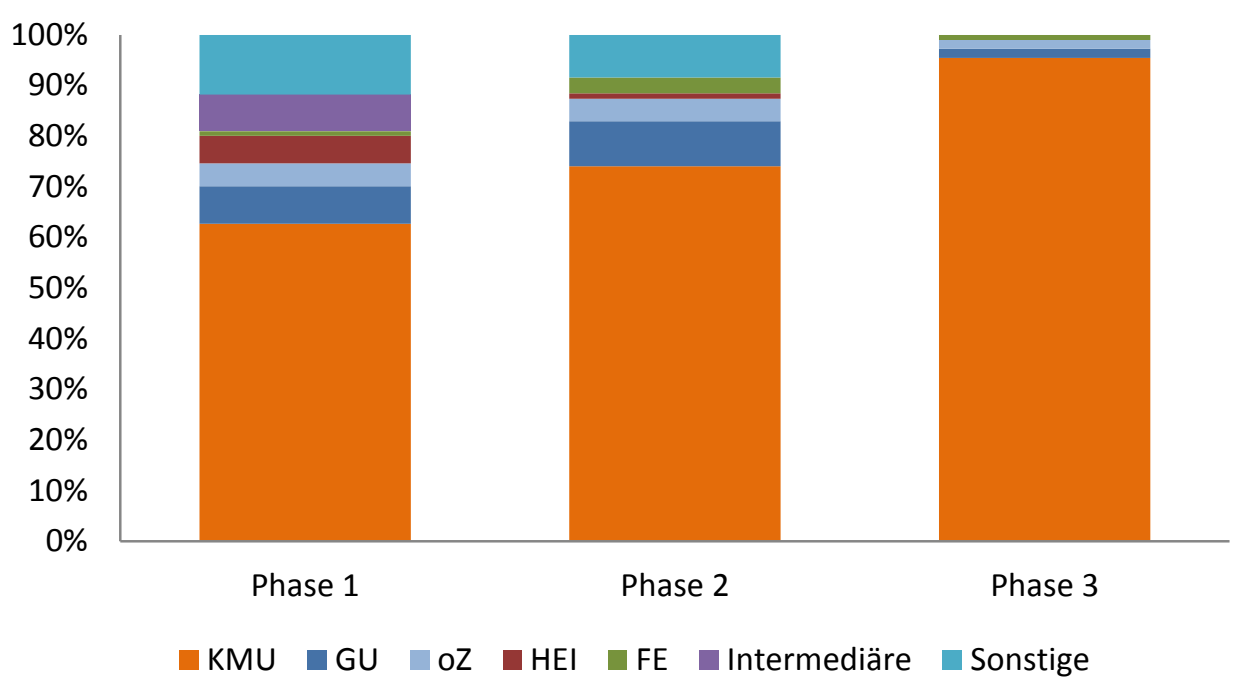

N = 159 für Phase 1, 71 für Phase 2 und 57 für Phase 3

$\mathrm{KMU}=$ kleine und mittlere Unternehmen, $\mathrm{GU}=$ Großunternehmen, oZ = Unternehmen ohne $\mathrm{KMU} / \mathrm{GU}$ Zuordnung, $\mathrm{HEI}=$ Hochschulen, $\mathrm{FE}=$ Forschungseinrichtungen

Quelle: FFG, eigene Darstellung.

Der gesamte Anteil von Organisationen, für die ihr AT:net Projekt die erste Förderung durch die FFG darstellte, lag in der ersten Phase bei $47 \%$, in Phase 2 bei $51 \%$ und in der dritten Phase bei $37 \%$. Auch hier stellen KMU die Mehrheit (siehe Abbildung 2). Der hohe gesamte Anteil von KMU sowie ihre Dominanz bezüglich der FFG Ersteinreicher leiten sich unmittelbar aus der Ausrichtung des Programms bzw. seiner KMU Orientierung ab. 
Abbildung 2 In AT:net geförderte Organisationen mit FFG Erstförderung nach Organisationstypen und Programmphasen, in Prozent aller Fördernehmer

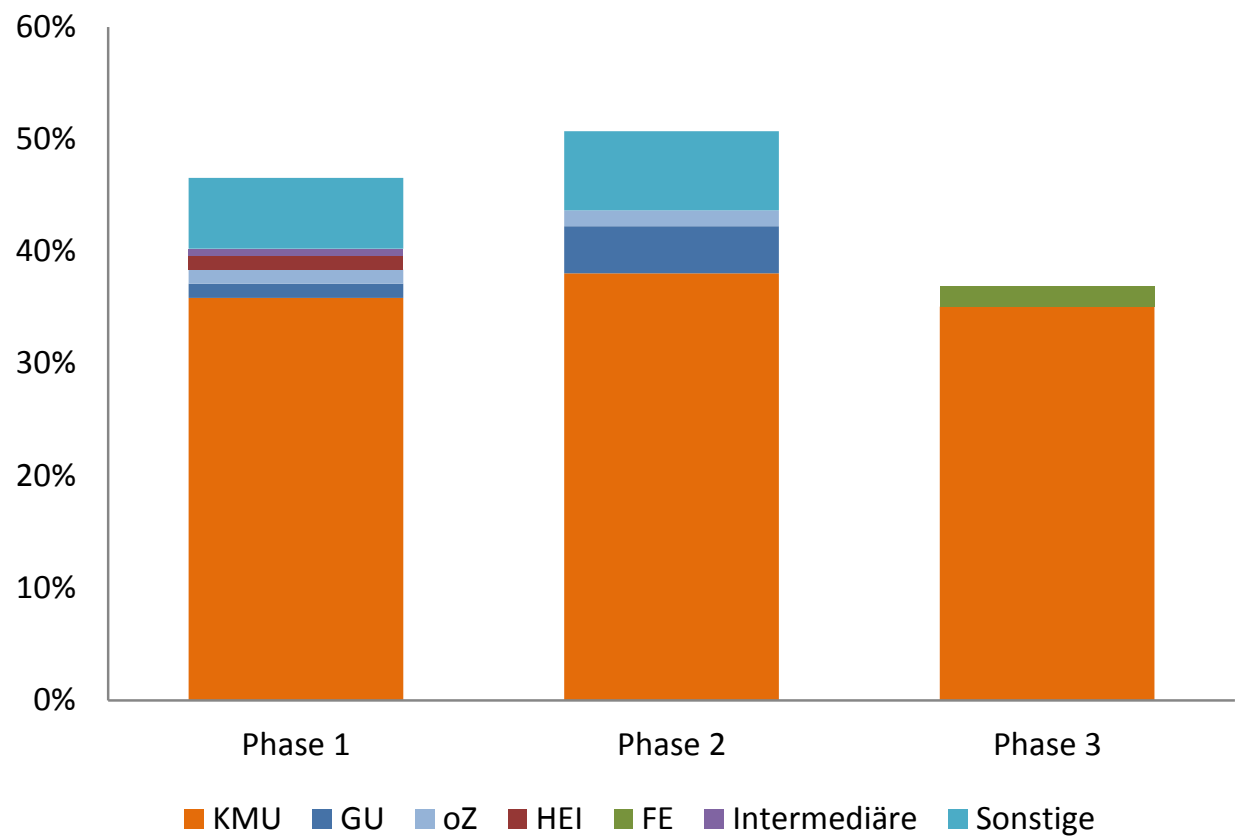

$\mathrm{N}=159$ für Phase 1, 71 für Phase 2 und 57 für Phase 3

$\mathrm{KMU}=$ kleine und mittlere Unternehmen, $\mathrm{GU}=$ Großunternehmen, oZ = Unternehmen ohne KMU/GU Zuordnung, $\mathrm{HEI}=$ Hochschulen, $\mathrm{FE}=$ Forschungseinrichtungen

Quelle: FFG, eigene Darstellung.

Der deutlich kleinere Anteil der Projekte waren bzw. sind Kooperationsprojekte (30 von 232), wovon die meisten (23) durch KMU koordiniert wurden/werden. Mehr als die Hälfte der Kooperationsprojekte wurden von zwei Organisationen gemeinsam durchgeführt, ein Drittel von drei und etwas mehr als $10 \%$ von 4 Organisationen. In einem Fall bestand das Konsortium aus 15 Organisationen.

Das Programm AT:net wurde insbesondere in den ersten Jahren in mehreren Informationstagen bzw. Roadshows vor allem in den Bundesländern beworben und vorgestellt. Die Roadshows haben in den Jahren 2008 (6 Informationstage), 2009 (5 Informationstage) und 2010 (2 Informationstage) stattgefunden. In den folgenden Jahren 2011-13 hat es diese Informationstage nicht gegeben, allerdings wurde das Programm weiterhin aktiv beworben, vor allem 2012 im Rahmen der IKT Auftaktveranstaltung zum Relaunch des zentralen IKT Förderprogramms „IKT der Zukunft“ sowie auf dem „Pioneers Festival“, der zentralen Veranstaltung der österreichischen IKT start-up Szene. Darüber hinaus wurden Netzwerkpartnern und Multiplikatoren wie regionalen Wirtschafts- und Technologieagenturen, Kammern und Interessenverbänden Informationspakete zu den aktuellen Calls zur Verfügung gestellt. 
Bereits in der Zwischenevaluierung des Programms wurde festgestellt, dass der Einschätzung des Programmmanagements zufolge diese bewusstseinsschaffenden Aktivitäten einen deutlichen Effekt auf die Anzahl der Einreichungen hatten. Dies schien besonders deutlich im Vergleich der ersten beiden Ausschreibungen in Phase 1 und der dritten Ausschreibung, die von insgesamt 6 Informationstagen in unterschiedlichen Bundesländern vorbereitet wurden. Allerdings haben diese Mobilisierungseffekte über den Programmverlauf stark nachgelassen bis hin zu einer lediglich 43\%igen Vergabe der maximal verfügbaren Mittel in der achten Ausschreibung (siehe Abbildung 3). Der 2012 vorgenommene Strategiewechsel mit Hinblick auf die Begleitmaßnahmen, also weg von regionalen Veranstaltungen zu eher technologie- bzw. branchenspezifischen Veranstaltungen, hat nicht nur die Anzahl der genehmigten Projekte erhöht (siehe Abbildung 4), sondern führte sogar zu einer gesamten Mittelzusage oberhalb der verfügbaren Mittel in der elften Ausschreibung (die beantragten Fördermittel lagen für die beiden Ausschreibungen in 2013 mit insgesamt $€$ 5,3 Millionen auch deutlich über den insgesamt 2,7 Millionen zur Verfügung stehenden). 
Abbildung 3 Mittelausschöpfung des Programms nach Phasen und Ausschreibungen

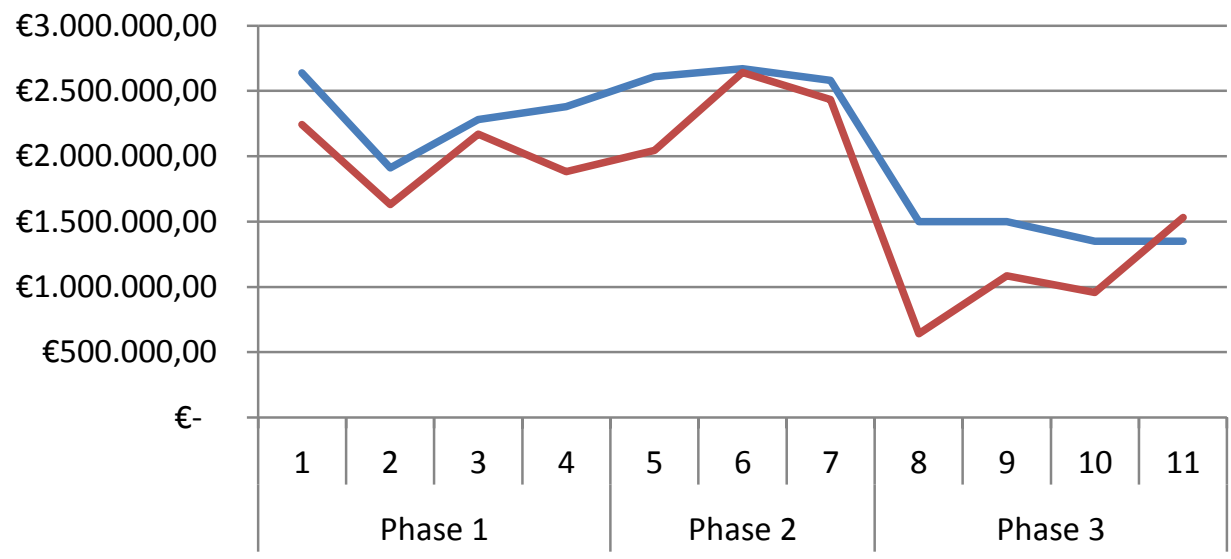

Maximale Mittelbindung $\quad$ Maximale Zusage

$N=232($ Phase $1=114$, Phase $2=63$, Phase $3=55)$

Quelle: FFG, eigene Darstellung

Abbildung 4 Eingereichte und genehmigte Projekte nach Phasen und Ausschreibungen

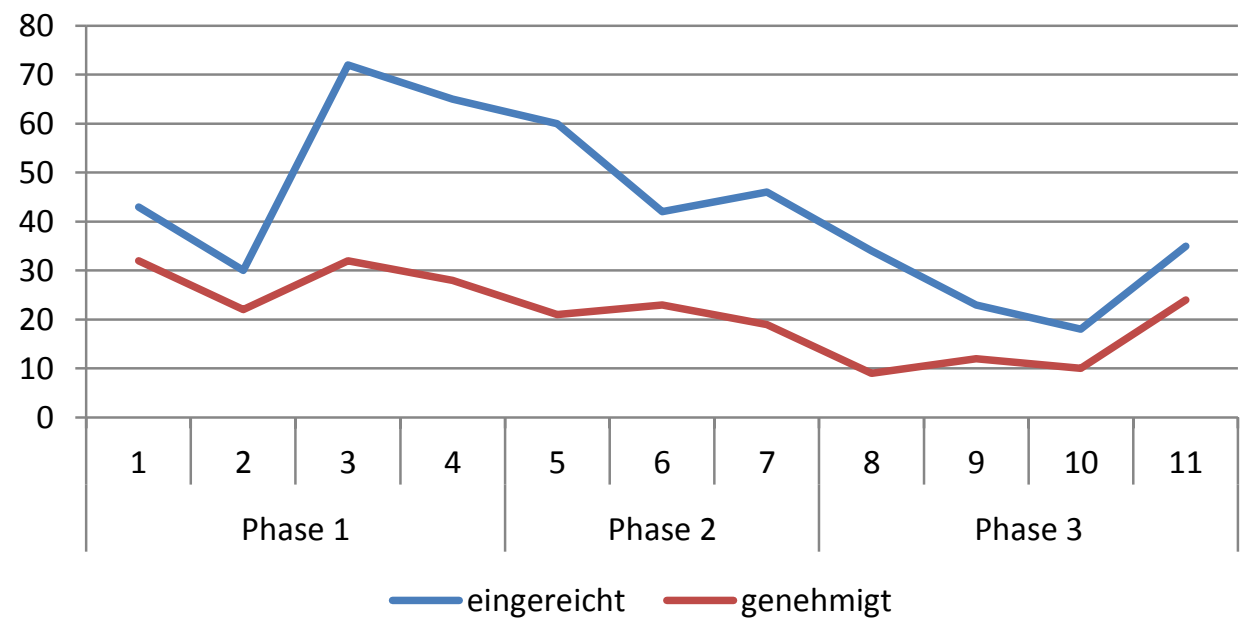

$N=232($ Phase $1=114$, Phase $2=63$, Phase $3=55)$

Quelle: FFG, eigene Darstellung.

Mit dem Wechsel im Programmmanagement wurde nicht nur die Bewerbungs- und Informationsstrategie geändert, sondern auch die Erfordernisse der Mittelbeantragung insofern erhöht, als das potenzielle Fördernehmer ihre Projekte hinsichtlich ihrer Struktur (d.h. Arbeitspakete und Meilensteine) bereits in den Anträgen detaillierter planen und darstellen. Neben einer vom Programmmanagement beobachte- 
ten, stärkeren kommerziellen Ausrichtung der Projekte, lässt sich aus den Monitoringdaten der FFG vor allem eine steigende Erfolgsquote bei der Einreichung (siehe Abbildung 5) erkennen, die allerdings unter den sehr hohen Werten der ersten und zweiten Ausschreibung liegt.

Abbildung 5 Erfolgsquote der eingereichten Projekte nach Phasen und Ausschreibungen

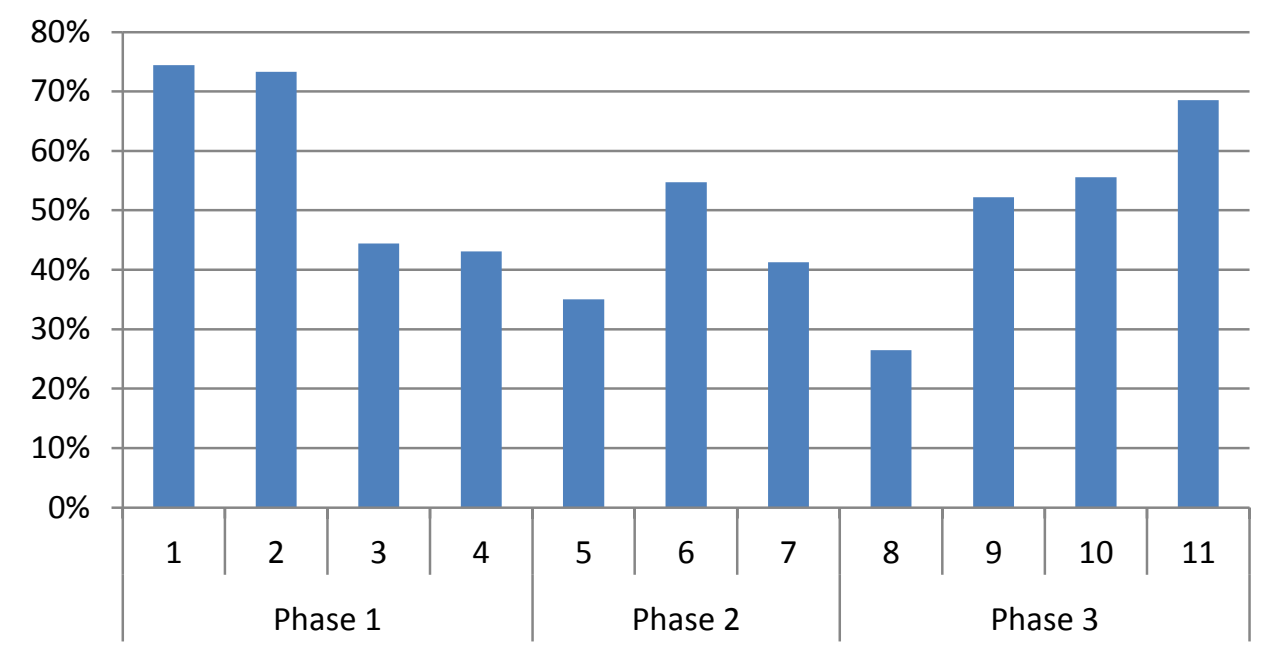

$\mathrm{N}=232($ Phase $1=114$, Phase $2=63$, Phase $3=55)$

Quelle: FFG, eigene Darstellung. 


\section{FFG-Beteiligung der in AT:net geförderten Organisationen}

Dieses Kapitel widmet sich der Fragestellung, inwieweit Forschung (und damit Forschungsförderung) für die in AT:net geförderten Organisationen (d.h. vor allem Unternehmen) und ihre konkreten Projekte relevant ist. Dazu geben vor allem die Analysen der Verbindungen zu geförderten Projekten in anderen FFG Programmen Hinweise darauf, inwieweit AT:net zusätzlich zur eigenen Themenvielfalt auf einer ebenso breiten Basis geförderter Organisationen hinsichtlich weiterer Forschungs- und Entwicklungsprojekte steht. Für die Evaluierung des Programms AT:net ist von Bedeutung, wie die entsprechend geförderten Organisationen möglicherweise (in Form von geförderten Projekten) an anderen FFGFörderprogrammen beteiligt sind, d.h. welche Förderprogramme von den Organisationen außerdem genutzt werden. Zum einen soll diese Analyse offenlegen, welche Querverbindungen es zwischen AT:net und den anderen Programmen gibt und zum anderen sollen die Auswertungen Hinweise darauf liefern, welche thematischen Schwerpunkte oder Profile die geförderten Organisationen hinsichtlich ihrer geförderten Forschungs- und Entwicklungsprojekte aufweisen.

Insgesamt haben die $234^{1}$ (in 208 AT:net Projekten) geförderten Organisationen seit 20011.500 weitere Projektbeteiligungen (davon 94 Unternehmen mit insgesamt 537) in anderen FFG-Programmen aufzuweisen. Die durchschnittliche Anzahl (Median) je Organisation liegt bei 90 Beteiligungen, wobei die Bandbreite von einer bis 741 Beteiligungen reicht. Diese Statistik wird jedoch durch Hochschulen und vor allem außeruniversitäre Forschungseinrichtungen dominiert und damit verzerrt. Betrachtet man nur die Unternehmen (zumal diese auch die Zielgruppe des Programms AT:net darstellen), dann liegt der Median der Beteiligungen bei drei mit einer Spannbreite von einer bis 47 Beteiligungen.

Über alle Organisationen hinweg haben die Programme Innovationsscheck (228; Unternehmen: 59), Talente (227; 70) und Basisprogramme $(216 ; 170)$ die meisten Beteiligungen aufzuweisen. Diese Gesamtverteilung macht deutlich, dass die Organisationen (die ja in erster Linie Unternehmen sind) vor allem niedrigschwellige und relativ marktnahe Förderangebote nutzen. Weiterhin lässt sich erkennen, dass die in AT:net geförderten Unternehmen insgesamt andere Nutzungsprofile aufweisen als die Forschungseinrichtungen etc. Ihr Anteil an allen sonstigen FFG Programmbeteiligungen ist vor allem deutlich höher im Basisprogramm. Andere Fördernehmer (d.h. vor allem Forschungseinrichtungen und Hochschulen) nutzen hingegen deutlich stärker die Programme Innovationsscheck und Talente. Diese

${ }^{1}$ Die Beteiligungsdaten für die Fördernehmer der 11. Ausschreibung standen für die vorliegende Evaluierung (noch) nicht zur Verfügung. 
Verteilung lässt sich vor allem auf den deutlichen Unternehmensfokus der entsprechenden Programme zurückführen.

Abbildung 6 Beteiligungsprofil der in AT:net geförderten Organisationen in anderen FFG-Programmen auf Basis der Anzahl von geförderten Projekten 20012013, Programme mit 15 oder mehr Beteiligungen insgesamt

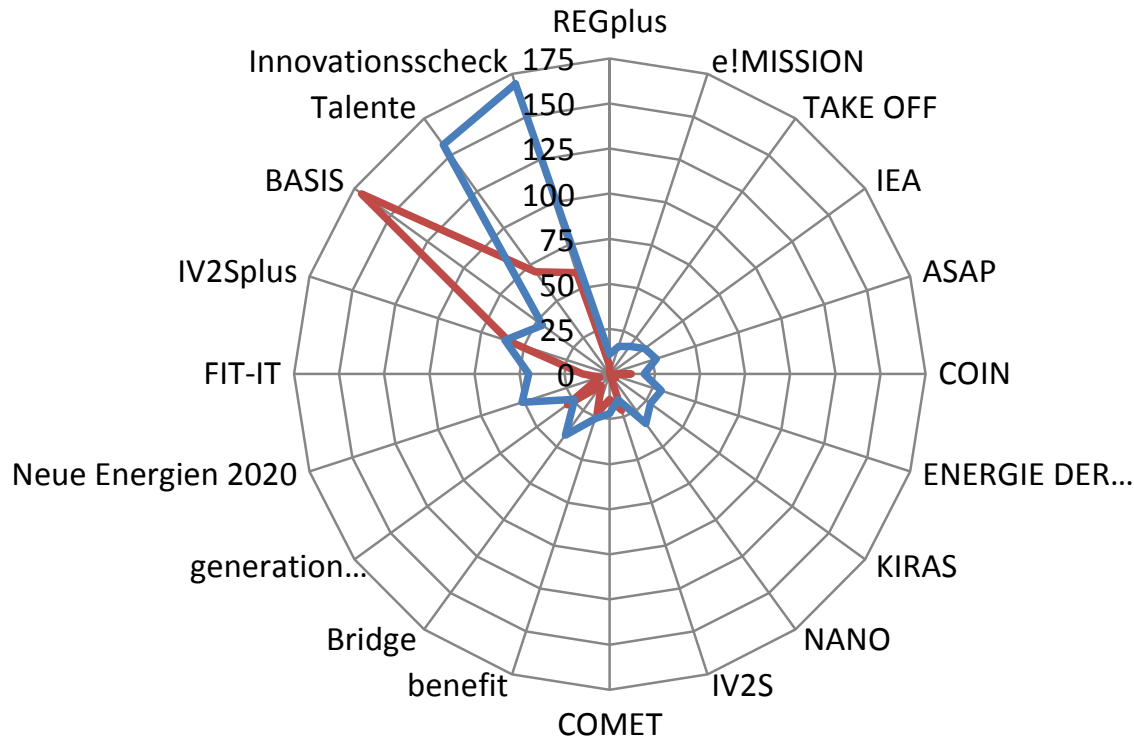

Unternehmen $\longrightarrow$ Andere

Ohne Fördernehmer aus der 11. Ausschreibung.

$n=1363$

Quelle: FFG, eigene Darstellung.

Die gleichen Auswertungen auf Basis einer Zusammenfassung inhaltlich ähnlicher Programme (siehe Abbildung 7) macht weiterhin deutlich, dass die gesamte Thematik der Förderung von Humanressourcen (nach verschiedenen Kriterien) deutlich relevanter für andere Fördernehmer als für Unternehmen ist, was allerdings ohnehin insofern zu erwarten ist, da diese Förderungen zumeist auf das Bildungsoder Forschungssystem abzielen. Eine spezifisch auf KMU abzielende Förderung wird unter den AT:net Fördernehmern in erster Linie nicht von Unternehmen genutzt, wobei die starke Dominanz des Innovationsschecks zu beachten ist gemeinsam mit dem Umstand, dass ein Großteil der Unternehmen in AT:net nicht zur Zielgruppe dieses Programms gehören. Des Weiteren ist zu beachten, dass etwa in verschiedenen thematischen Bereichen der Anteil von industrieller Forschung und Entwicklung stark unterschiedlich ist: z.B. Nanowissenschaften oder Energie mit einer nach wie vor starken Grundlagenforschungsorientierung gegenüber Mobilität und Verkehr. Ohne auf Basis der existierenden Daten für die spezifische the- 
matisch-technologische Ausrichtung der Fördernehmer in AT:net kontrollieren zu können, sind diese Unterschiede trotzdem sichtbar.

Abbildung 7 Beteiligungsprofil der in AT:net geförderten Organisationen in Clustern von Programmen auf Basis der Anzahl von geförderten Projekten 20012013

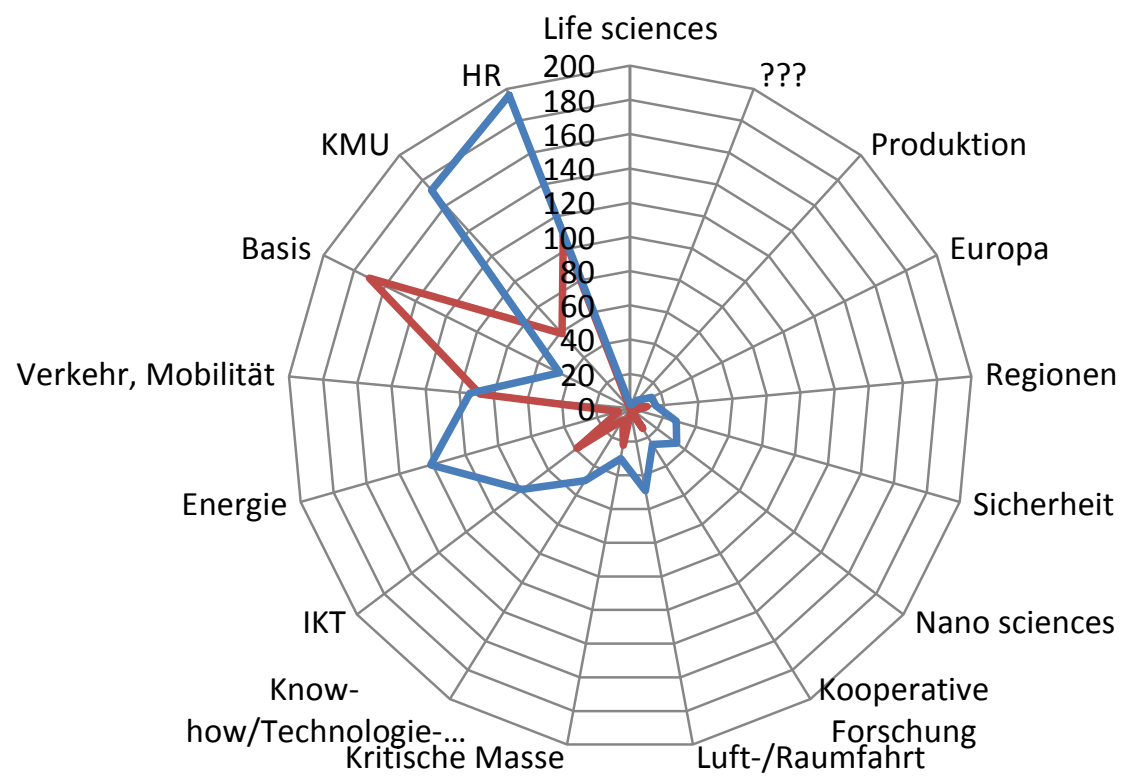

Unternehmen $\longrightarrow$ Andere

Ohne Fördernehmer aus der 11. Ausschreibung.

$n=1363$

Quelle: FFG, eigene Darstellung

Eine zusätzliche Interpretationsebene, ohne im Zusammenhang mit der vorliegenden Analyse die Komplexität der Situation vollständig berücksichtigen zu können, stellt die folgende Abbildung 8 dar: die Anzahl der Beteiligungen von in AT:net geförderten Unternehmen in Clustern von FFG-Programmen wurde um einen Gewichtungsfaktor erweitert, der ihre geringere Anzahl an allen AT:net Fördernehmern auszugleichen sucht. ${ }^{2}$

${ }^{2}$ Der gewählte Gewichtungsfaktor kann allerdings nicht helfen, die unterschiedliche Anzahl von Projekten je Organisation oder für andere, tieferliegende Unterschiede kontrollieren. Der Ansatz kann daher nur als näherungsweise Kontrolle verstanden werden. 
Diese Analyse vermag einige der zuvor getroffenen Aussagen zu ergänzen und zwar dahingehend, dass relativ zu ihrer Anzahl als AT:net Fördernehmer die Unternehmen sehr wohl Programme aus dem Bereich Humanressourcen in Anspruch nehmen und, was für die Frage des Forschungsgehalts von AT:net bzw. der Forschungsorientierung von hierin geförderten Unternehmen wichtiger ist, sehr stark im Verkehrs- und Mobilitätsbereich in geförderten Forschungs- und Entwicklungsprojekten engagiert sind. Auch die Relevanz der IKT-spezifischen Fördermaßnahmen für die Unternehmen wird in dieser Darstellung deutlicher.

\section{Abbildung 8 Beteiligung der in AT:net geförderten Organisationen in Clustern von} Programmen, Anzahl von geförderten Projekten 2001-2013

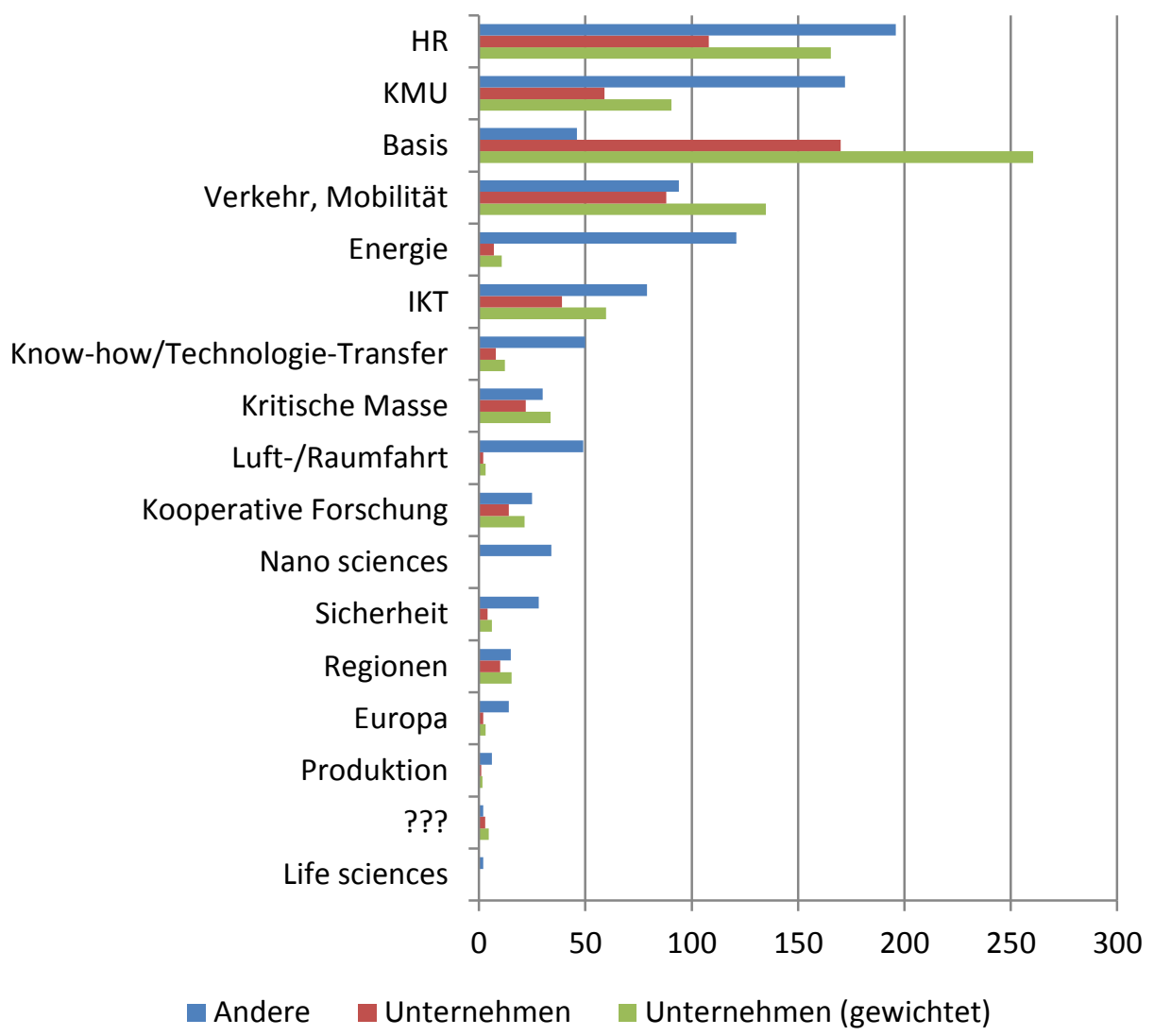

Ohne Fördernehmer aus der 11. Ausschreibung.

Der Gewichtungsfaktor der Programmbeteiligungen (Cluster von Programmen) der geförderten Unternehmen liegt bei 1,53 (Anzahl der anderen Fördernehmer/Anzahl der Unternehmen).

??? umfasst Programme, die nicht geclustert werden konnten.

$N=1500$

Quelle: FFG, eigene Darstellung.

Weitere Analysen auf Basis der beteiligten Unternehmen verdeutlichen weitere Muster in der Nutzung der FFG-Programme: Die 15 Unternehmen mit jeweils mehr 
als 10 Projekten in allen FFG-Programmen seit 2001 vereinen in Summe 291 solcher Projekte oder 19,4\% aller Projektbeteiligungen von in AT:net geförderten Organisationen (bzw. 54\% aller Beteiligungen durch Unternehmen) auf sich. Demzufolge sind all jene Programme in der folgenden Betrachtung von Bedeutung, in denen die prozentuale Beteiligung entweder deutlich höher oder deutlich niedriger liegt. Dazu werden nur jene Programme mit insgesamt 10 oder mehr Beteiligungen analysiert. In den folgenden Programmen vereinen die relativ häufig geförderten 15 Unternehmen (d.h. mit mehr als 10 Projekten) mehr als die zu erwartenden 20\% der Beteiligungen auf sich: generation innovation Praktika (47\%), IV2S/IV2Splus (38,5\%), Basisprogramme (36,1\%), COMET (25\%) und Talente $(24,7 \%)$. Im Gegenzug sind diese Unternehmen weniger häufig aktiv in den folgenden Programmen: COIN, K-ind/K-net, benefit, FIT-IT/IKT der Zukunft, Mobilität der Zukunft, KIRAS, FEMtech, Bridge, Innovationsscheck. Demzufolge sind Unternehmen mit nur wenigen FFG-Projekten in Summe aktiver in Neue Energien 2020, NANO, ENERGIE DER ZUKUNFT, ASAP, IEA, e!MISSION, REGplus und anderen. Der Unterschied ist am deutlichsten im Programm Innovationsscheck, in dem nur 7,5\% der 228 Projektbeteiligungen (29\% nur auf die Unternehmen insgesamt bezogen) auf jene Unternehmen fallen, die 10 oder mehr Beteiligungen insgesamt aufweisen. Erweitert man die Analyse auf Unternehmen mit mehr als 5 Projekten in FFGProgrammen (in Summe 30 Unternehmen mit 391 Projekten), ändert sich das Gesamtbild nur wenig, d.h. der Eindruck bleibt bestehen bis auf die Ergänzung um das Förderprogramm benefit. 
Abbildung 9 Anteil der häufig geförderten Unternehmen an den Programmbeteiligungen aller in AT:net geförderten Organisationen, in \% aller Beteiligungen an Programmen mit insgesamt mehr als 10 Beteiligungen

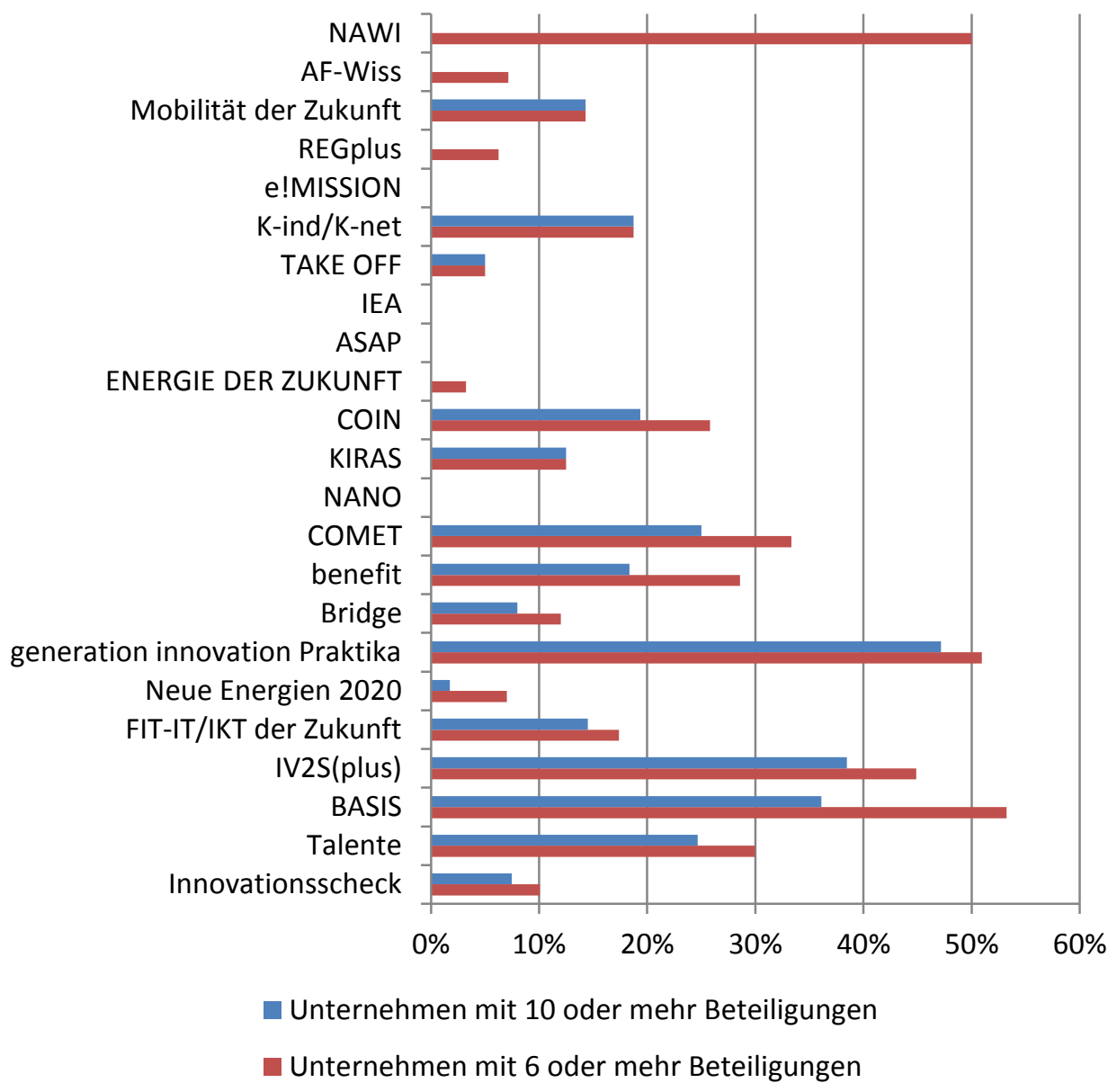

Ohne Fördernehmer aus der 11. Ausschreibung.

$n=391$

Quelle: FFG, eigene Darstellung.

Je nachdem in welcher Form die Programme nach Ähnlichkeit bzw. Zusammengehörigkeit zusammengefasst werden, ist zu erkennen, welche Förderthemen oder -bereiche für Unternehmen aus AT:net besonders relevant sind.

Die Unternehmen mit den meisten Beteiligungen (10 oder mehr) an FFGProgrammen zeigen einerseits vergleichbare Muster, andererseits individuelle Nutzungsprofile. Grundsätzlich wird deutlich, dass alle die Mehrheit ihrer Programmbeteiligungen auf wenige Programme fokussieren (im Durschnitt 5 Programme) und zusätzlich den Großteil ihrer geförderten Projekte in nur einem oder zwei FFG Programmen einreichen. Die Unterschiede sind am deutlichsten dahingehend, dass einige dieser Fördernehmer nahezu alle ihrer Beteiligungen in Programmen zur Unterstützung der Entwicklung von Humanressourcen wie generati- 
on innovation oder Talente haben, während andere klare thematische Fokussierungen aufweisen, insbesondere im Bereich Verkehr und Mobilität (IV2S, IV2Splus etc.). Während die Beteiligung an IKT-spezifischen Förderprogrammen vergleichsweise wenig ausgeprägt ist, stellen die Basisprogramme das wichtigste Förderinstrument dar (allerdings sind unter diesen 15 Unternehmen auch 4 ohne jede Beteiligung an den Basisprogrammen).

Abbildung 10 Beteiligungsprofil der Unternehmen mit 10 oder mehr FFG-Projekten, Anzahl der Beteiligungen an FFG-Förderprogrammen

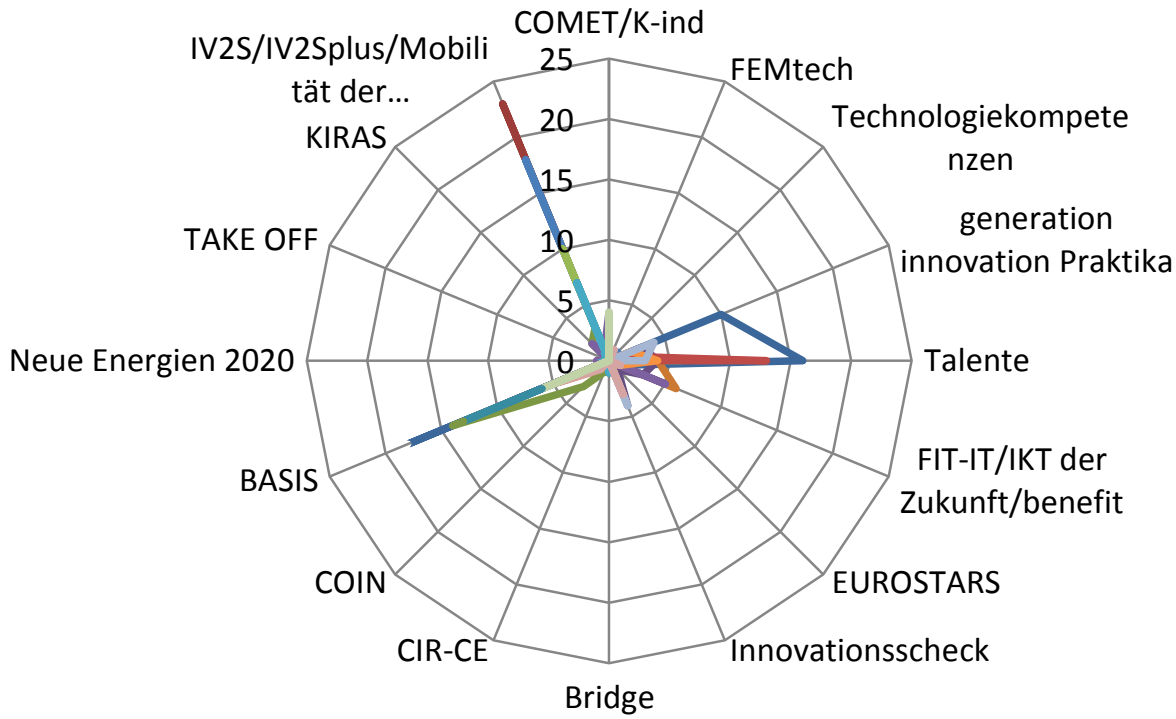

Ohne Fördernehmer aus der 11. Ausschreibung

$n=291$

Quelle: FFG, eigene Darstellung.

Vergleicht man die Beteiligungen im Sinne der relativen Bedeutung der verschiedenen Programme zwischen den Unternehmen mit 10 und mehr, 6-9 und 1-5 Programmbeteiligungen (siehe Abbildung 11) ergeben sich weitere Erkenntnisse. Förderungen im Bereich der Humanressourcen sind für jene Unternehmen mit 10 oder mehr Beteiligungen überproportional von Bedeutung, ebenso wie Programme aus dem Themenbereich Mobilität und Verkehr. Eher unspezifische, d.h. thematisch nicht eingeschränkte, Kooperationsförderung ist für diejenigen Organisationen deutlich bedeutender, die 6-9 Beteiligungen an FFG Programmen aufzuweisen haben. Eher KMU-spezifische Förderinstrumente wie Innovationsscheck und EUROSTARS sind wiederum vor allem für jene Fördernehmer mit 1-5 Programmbeteiligungen von Relevanz ebenso wie Programme aus dem Themenfeld Energie. 
Abbildung 11 Relative Bedeutung von Clustern von Programmen für AT:net Fördernehmer, Vergleich Unternehmen mit 10 oder mehr Beteiligungen, 6-9 und 1-5 Beteiligungen, prozentualer Anteil der Beteiligungen an allen Beteiligungen der jeweiligen Gruppe

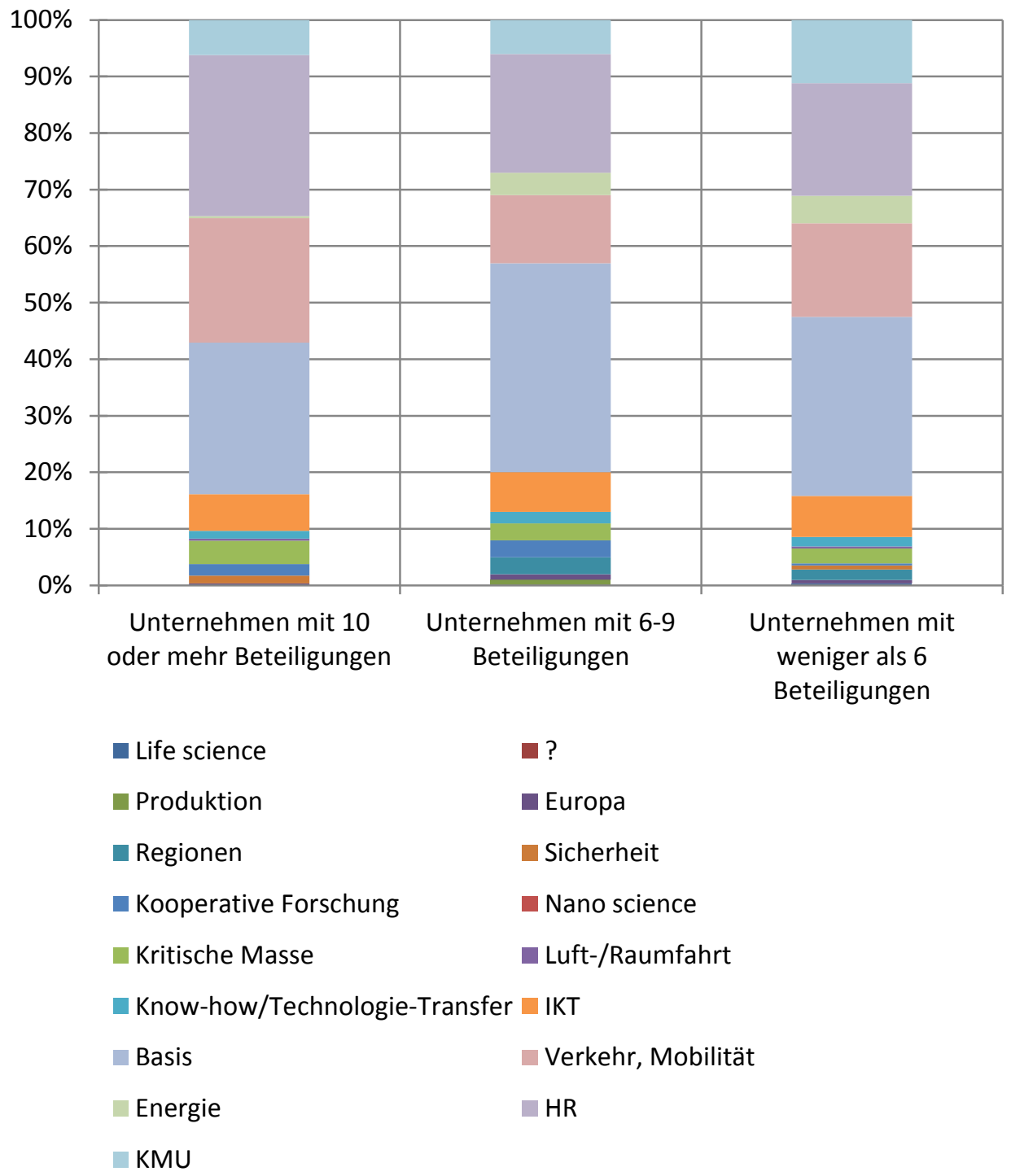

Ohne Fördernehmer aus der 11. Ausschreibung.

? umfasst Programme, die nicht geclustert werden konnten.

$n=928$

Quelle: FFG, eigene Darstellung.

Dies verdeutlicht zudem, dass Programme aus dem Bereich Humanressourcen sehr wohl von großer, relativer Bedeutung für jene Fördernehmer mit vergleichsweise wenigen Programmbeteiligungen sind, ebenso wie thematisch unspezifische 
Kooperationsprojekte. Abbildung 12 zeigt die prinzipielle Ähnlichkeit der (absoluten) Beteiligungsprofile.

Abbildung 12 Absolute Bedeutung von Clustern von Programmen für AT:net Fördernehmer, Vergleich Unternehmen mit 10 oder mehr Beteiligungen, 6-9 und 1-5 Beteiligungen

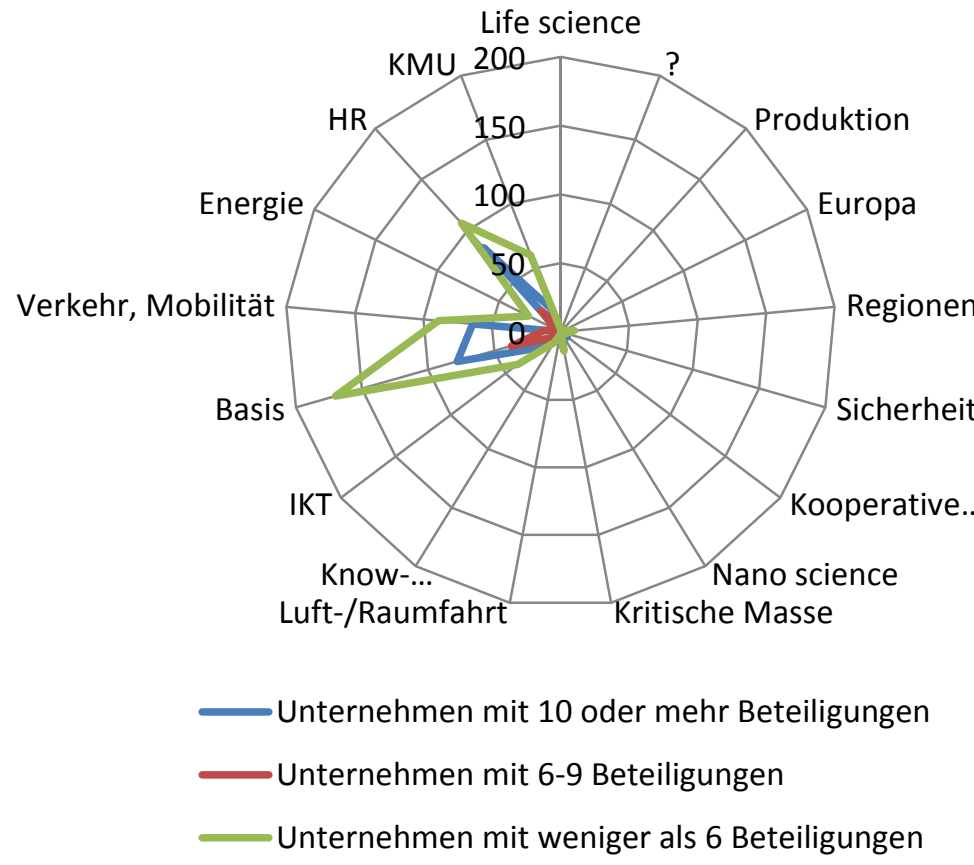

Ohne Fördernehmer aus der 11. Ausschreibung.

? umfasst Programme, die nicht geclustert werden konnten.

$n=928$

Quelle: FFG, eigene Darstellung.

Insgesamt ist offenkundig, dass die AT:net Fördernehmer für ein relativ breites Themenspektrum hinsichtlich ihrer (durch die FFG geförderten) Forschungs- und Innovationsaktivitäten stehen, wobei sich deutliche Spezialisierungen im Themenfeld Energie und Verkehr/Mobilität sowie in geringerem Ausmaß IKT ergeben. Von größerer Bedeutung sind jedoch Instrumente, die vor allem auf Humanressourcen sowie die Unterstützung von KMU (hier insbesondere der Innovationsscheck) abzielen. Für die allgemeine Forschungsintensität der geförderten Organisationen lässt sich daraus ableiten, dass AT:net neben deutlich forschungsintensiven Organisationen vielfach solche unterstützt, die sich außerhalb der klassischen Innovationsprojekte bewegen, was auch den relativ hohen Anteil von Organisationen ohne vorherige Fördererfahrung (FFG) erklärt. 


\section{Breitbandinfrastruktur und -nutzung in Ös- terreich}

Das Förderprogramm austrian electronic network steht unter dem Motto „Bandbreite für Breitband" und beinhaltet eine Reihe von Zielsetzungen, die im Zusammenhang mit der Einführung von qualitativen und innovativen Breitbanddiensten und -anwendungen stehen. Darunter fallen auch der Breitbandausbau sowie die Unterstützung qualitativer, innovativer, preiswerter und flächendeckend verfügbarer Breitbandzugänge. Obwohl es nicht möglich ist, aus den entsprechende verfügbaren Sekundärstatistiken Kausalität zwischen der Entwicklung bestimmter Indikatoren und der in AT:net geförderten Projekte herzustellen, werden im Folgenden Berichte und Daten analysiert, um den Status und die Entwicklung des Breitbandinternets in Österreich einordnen zu können.

\subsection{Haushalte}

Daten zur Nutzung und Verfügbarkeit von Breitbandinternet werden von der Statistik Austria seit 2002 erhoben. In diesem Zeitraum ist insgesamt ein starkes Wachstum der Internetanbindung und des Breitbandzugangs durch Haushalte sowie Einzelpersonen zu beobachten. So stieg die Anzahl der Haushalte mit Internetzugang von $33,5 \%$ auf $80,9 \%$ und der entsprechende Anteil von Haushalten mit Breibandanbindung von $27,5 \%$ (bezogen auf alle Haushalte: 10,3\%) auf $98,6 \%$ (79,8\%). Für die österreichischen Haushalte lässt sich vor allem für die Jahre 2005-2007 eine insgesamt sehr dynamische Gesamtentwicklung erkennen. Mit dem Erreichen der 75\% Marke im Jahr 2011 ist allerdings eine Sättigung erkennbar, d.h. die Wachstumsraten liegen deutlich unter dem Durchschnitt der Vorjahre. Gleiches gilt für die Breitbandversorgung, die jedoch seit 2013 ohnehin bei nahezu 100\% aller an das Internet angeschlossenen österreichischen Haushalte liegt (siehe Abbildung 13 bzw. Abbildung 14). 
Abbildung 13 Status - Österreichische Haushalte mit Interzugang und Breitbandverbindung in Prozent aller Haushalte bzw. in Prozent aller Haushalte mit Internetzugang, 2002-2013

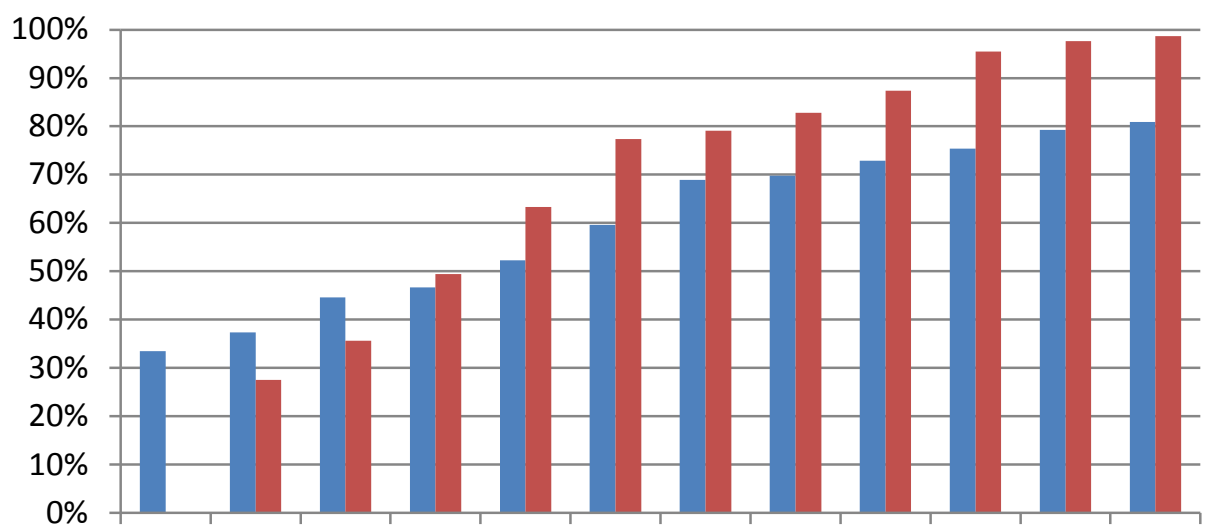

200220032004200520062007200820092010201120122013

Haushalte mit Internetzugang

davon mit Haushalte mit Breitbandanbindung

Quelle: Statistik Austria, eigene Darstellung.

Abbildung 14 Entwicklungsdynamik - Österreichische Haushalte mit Interzugang und Breitbandverbindung, Veränderung zum Vorjahr in Prozentpunkten, 2003-2013

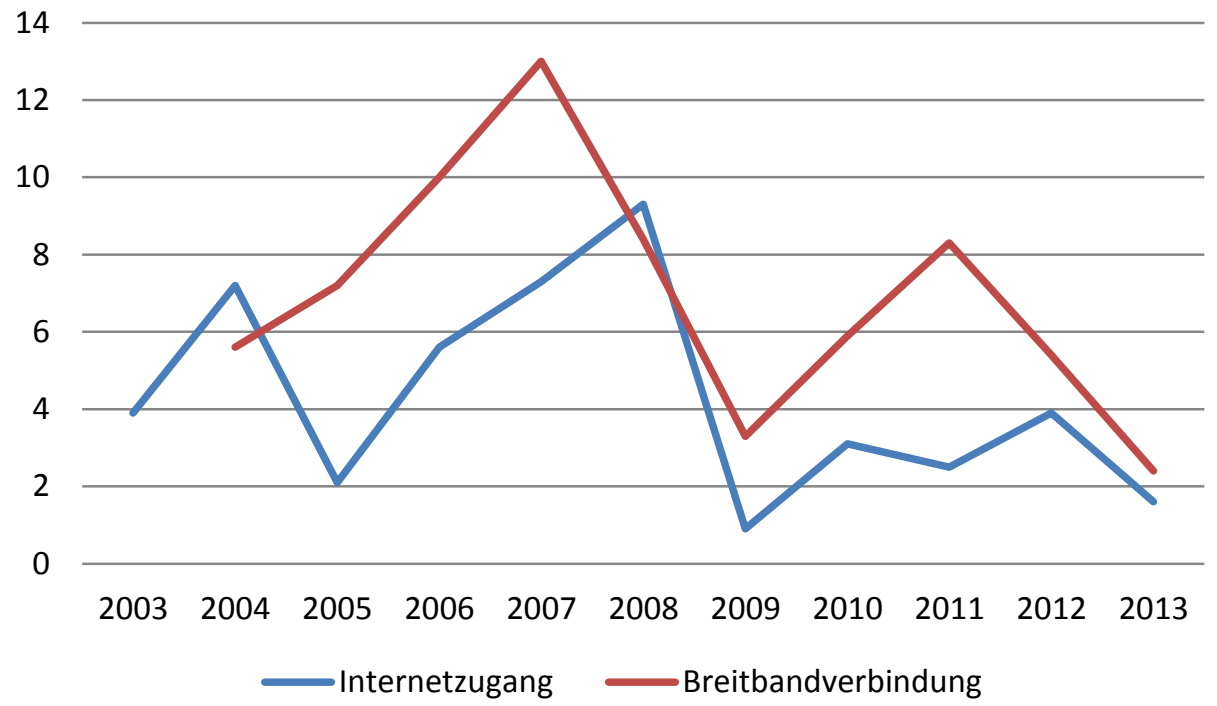

Quelle: Statistik Austria, eigene Darstellung.

Dabei ist auffällig, dass Mehrpersonenhaushalte (d.h. 3 oder mehr Personen im jeweiligen Haushalt) von dieser Entwicklung stärker profitieren als andere (siehe Abbildung 15). 
Abbildung 15 Status - Österreichische Haushalte mit Breitbandanbindung in Prozent aller Haushalte nach Haushaltsgröße, 2002-2013

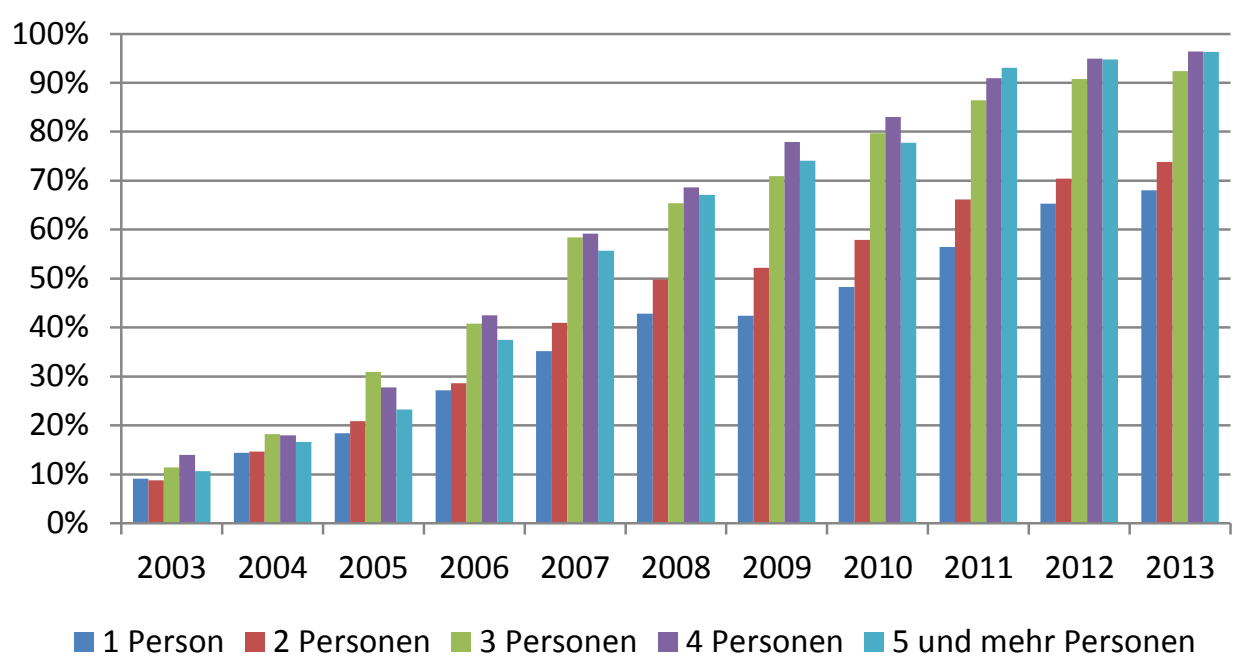

Quelle: Statistik Austria, eigene Darstellung.

Insgesamt verteilen sich 2013 die Internetzugänge der österreichischen Haushalte nahezu gleichmäßig auf fixe und mobile Zugänge, wobei Ein-Personen-Haushalte über mehr mobile als fixe Zugänge verfügen, während für alle anderen Haushaltsgrößen das Verhältnis umgekehrt ist und feste Breitbandzugänge zum Teil deutlich häufiger sind (siehe Abbildung 16). 
Abbildung 16 Status - Österreichische Haushalte mit Breitbandanbindung, Art der Anbindung in Prozent aller Haushalte nach Haushaltsgröße, 2013

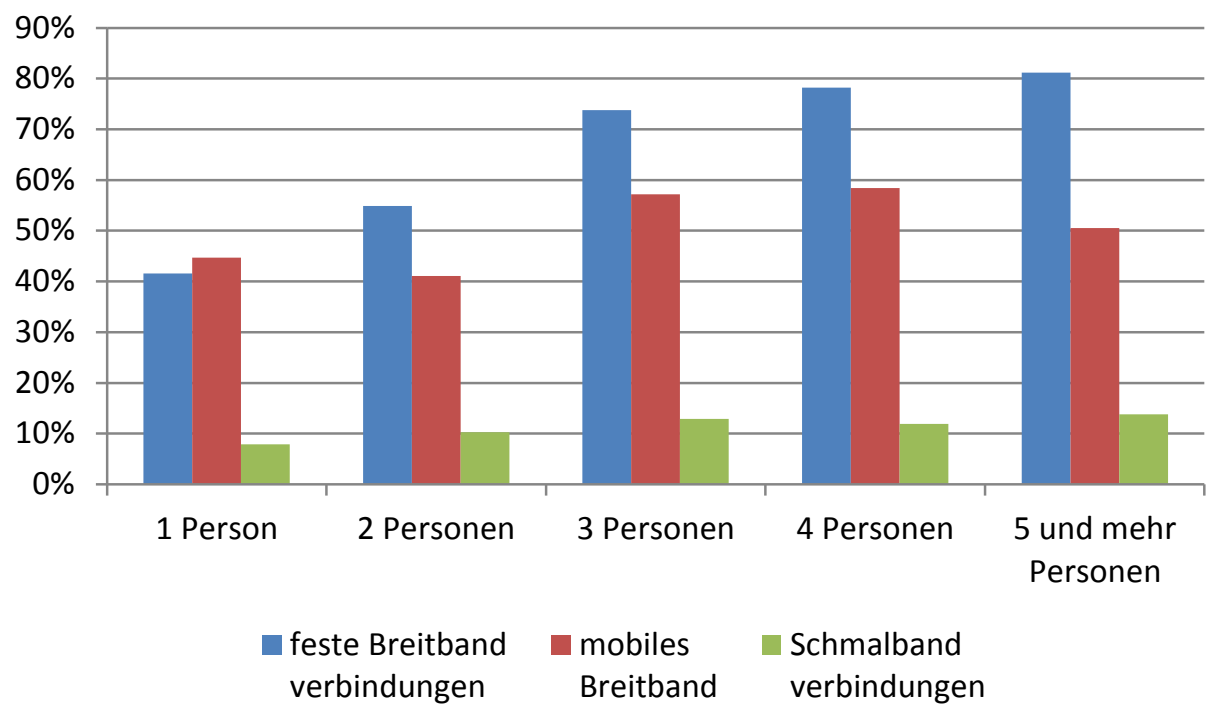

Quelle: Statistik Austria, eigene Darstellung.

Insgesamt wird die große Bedeutung mobiler Internetzugänge deutlich, genau wie die Parallelnutzung fester und mobiler Breitbandverbindungen. Hinsichtlich der genutzten Endgeräte ist festzuhalten, dass 56\% Mobiltelefone nutzen und etwa ein Drittel tragbare Computer.

Gemäß den Analysen der Statistik Austria ${ }^{3}$ gibt es keine signifikanten Unterschiede zwischen den Bundesländern. Wien, Vorarlberg und Salzburg lagen 2012 demnach über dem österreichischen Durchschnitt der Haushalte mit Breitbandanbindung, während Tirol und das Burgenland am deutlichsten darunter lagen. Die Verteilung auf mobile bzw. feste Zugänge ist ebenfalls nur wenig unterschiedlich.

Hinsichtlich der Internetnutzung nach Altersgruppen ist zunächst festzustellen, dass das Globalziel (das auch in AT:net relevant ist), den Anteil der InternetnutzerInnen auf $80 \%$ aller Österreicherlnnen zu erhöhen, erreicht wurde (siehe Abbildung 17). Im Sinne der Verringerung der digitalen Kluft ist das Gesamtergebnis allerdings deutlich weniger positiv, da etwa die Nutzung des Internets unter den 65-74 Jahren (also jener Gruppe, deren Inklusion vor dem Hintergrund einer alternden Gesellschaft relativ bedeutend, aber auch am wenigsten einfach zu erreichen ist) nicht nur insgesamt stark unterdurchschnittlich bleibt, sondern sogar leicht rückläufig zu sein scheint.

${ }^{3}$ Statistik Austria (Hg.), 2012: IKT-Einsatz in Haushalten. Einsatz von Informations- und Kommunikationstechnologien in Haushalten 2012, Wien. 


\section{Abbildung 17 Internetnutzung nach Altersgruppen, Entwicklung 2002-2013}

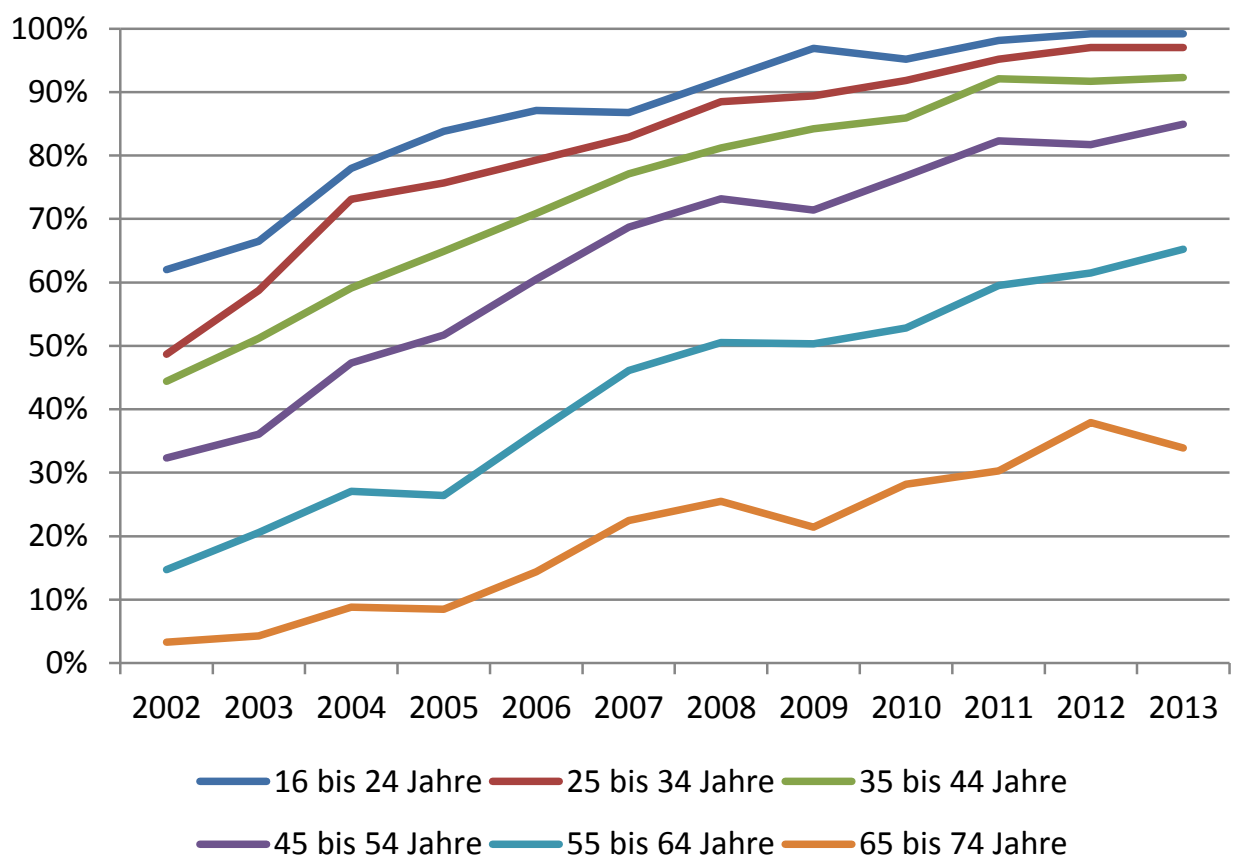

Quelle: Statistik Austria, eigene Darstellung.

Die für 2013 erhobene Nutzung des Internets (inklusive aller möglichen Zugänge und Verbindungstypen) betont die Bedeutung des Internets als Informationsquelle und als Zugang zu verschiedenen Dienstleistungen. So nutzen alle Internetnutzer die Möglichkeiten, sich zu Waren und Dienstleistungen zu informieren (das entspricht $83,4 \%$ aller Personen) und nahezu $70 \%$ aller Personen nutzen das Internet als allgemeine Wissensquelle (Nutzung von Wikis und anderen Nachschlagewerken). Unter den genutzten Dienstleistungen stechen hinsichtlich ihrer Bedeutung vor allem Internet-Banking, Online-Shopping sowie die Buchung von Reiseleistungen hervor. eGovernment in den drei erhobenen Ausprägungen Informationssuche, Herunterladen sowie Ausfüllen entsprechender Formulare ist deutlich weniger häufig. 
Abbildung 18 Status - Nutzungsverhalten österreichischer Internetnutzer nach Nutzungsart in Prozent aller Personen, 2013

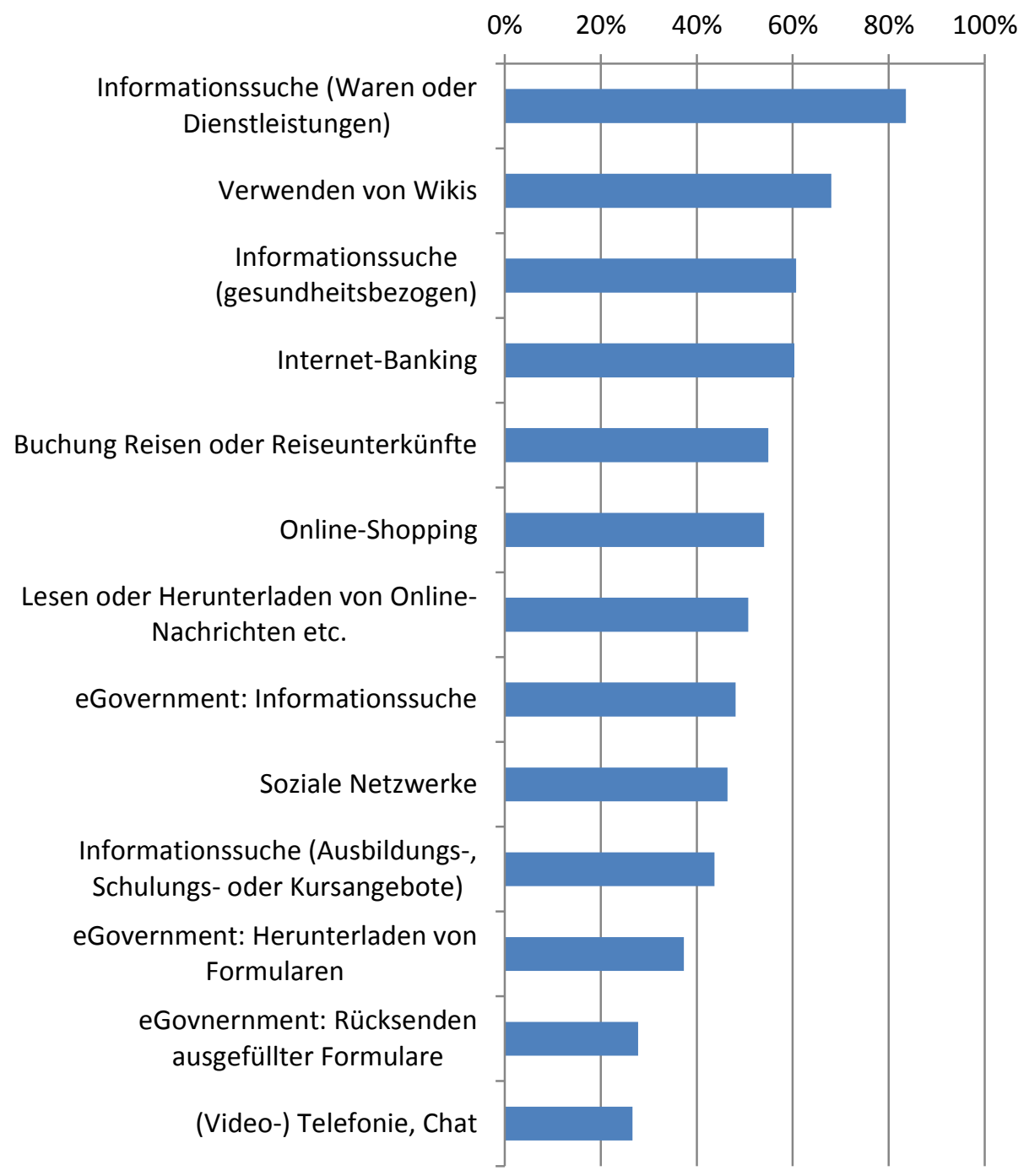

Quelle: Statistik Austria, eigene Darstellung.

\subsection{Unternehmen}

Informationen zur Internetnutzung und Breitbandanbindung der österreichischen Unternehmen stehen für die Jahre 2003 bis 2013 zur Verfügung. Insgesamt gilt, dass Unternehmen bereits deutlich länger als private Nutzer eine gesättigte Abdeckung mit Internetzugängen aufweisen. Während der Anteil dieser Unternehmen bereits seit 2006 bei über $97 \%$ liegt (und sich dementsprechend wenig dynamisch entwickelt hat), ist bei den Anschlüssen an Breitbandinfrastruktur eine ähnliche Entwicklung wie bei den privaten Haushalten zu beobachten, d.h. vor allem in den Jahren 2003-2008 und 2010/11 ist eine deutlich dynamische Entwicklung zu be- 
obachten. Insgesamt hat sich der Anteil der Unternehmen mit Internetzugang von 89,2\% (2003) auf 97,6\% erhöht. Der Anteil der Unternehmen mit Breitbandanbindung ist von $54,6 \%$ (48,7\% auf alle Unternehmen gerechnet) auf 95,7\% (93,4\%) gestiegen (siehe Abbildung 19 und Abbildung 20).

Abbildung 19 Status - Österreichische Unternehmen mit Interzugang und Breitbandanbindung in Prozent aller Unternehmen bzw. in Prozent aller Unternehmen mit Internetzugang, 2003-2013

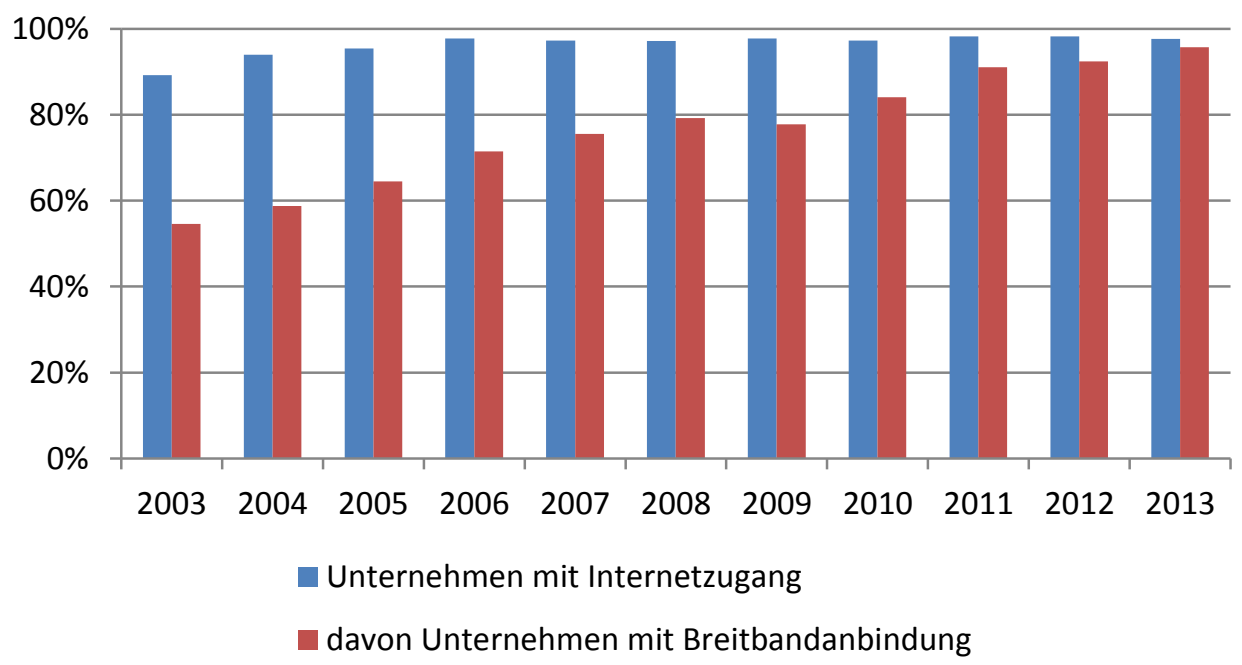

Quelle: Statistik Austria, eigene Darstellung.

Abbildung 20 Entwicklungsdynamik - Österreichische Unternehmen mit Interzugang und Breitbandverbindung, Veränderung zum Vorjahr in Prozentpunkten, 2004-2013

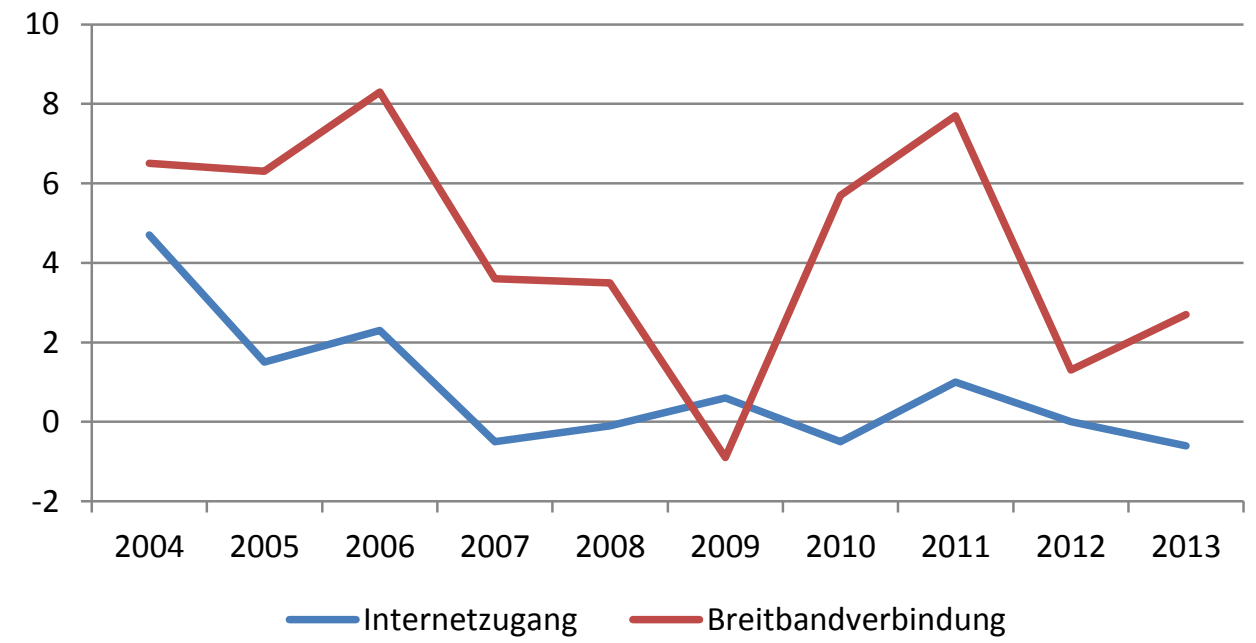

Quelle: Statistik Austria, eigene Darstellung.

Diese Entwicklung bedeutet auch, dass zwar mehr Unternehmen als Haushalte über einen Internetzugang und eine Anbindung an Breitbandinternet verfügen, bei 
letzteren prozentual gesehen die Breitbandanbindung jedoch höher ist $(98,6 \%$ gegenüber $95,7 \%$ ).

Hinsichtlich der Aktivitäten der Unternehmen im eGovernment Bereich wird aus den aktuellsten Daten (2012) deutlich, dass der Zugang zu entsprechenden Formularen bzw. Informationsquellen sowie das online Ausfüllen von Formularen von besonderer Bedeutung sind, wohingegen die Abwicklung vollständiger behördlicher Vorgänge sowie Teilnahme an eProcurement-Verfahren nur noch für etwa die Hälfte bzw. ein Fünftel aller Unternehmen mit eGovernment Aktivitäten relevant ist (siehe Abbildung 21). Dabei sind größere Unternehmen in allen Kategorien aktiver als kleine.

Abbildung 21 Status - Österreichische Unternehmen mit eGovernment Aktivitäten nach Größenklassen, in Prozent aller Unternehmen mit eGovernment Aktivitäten, 2012

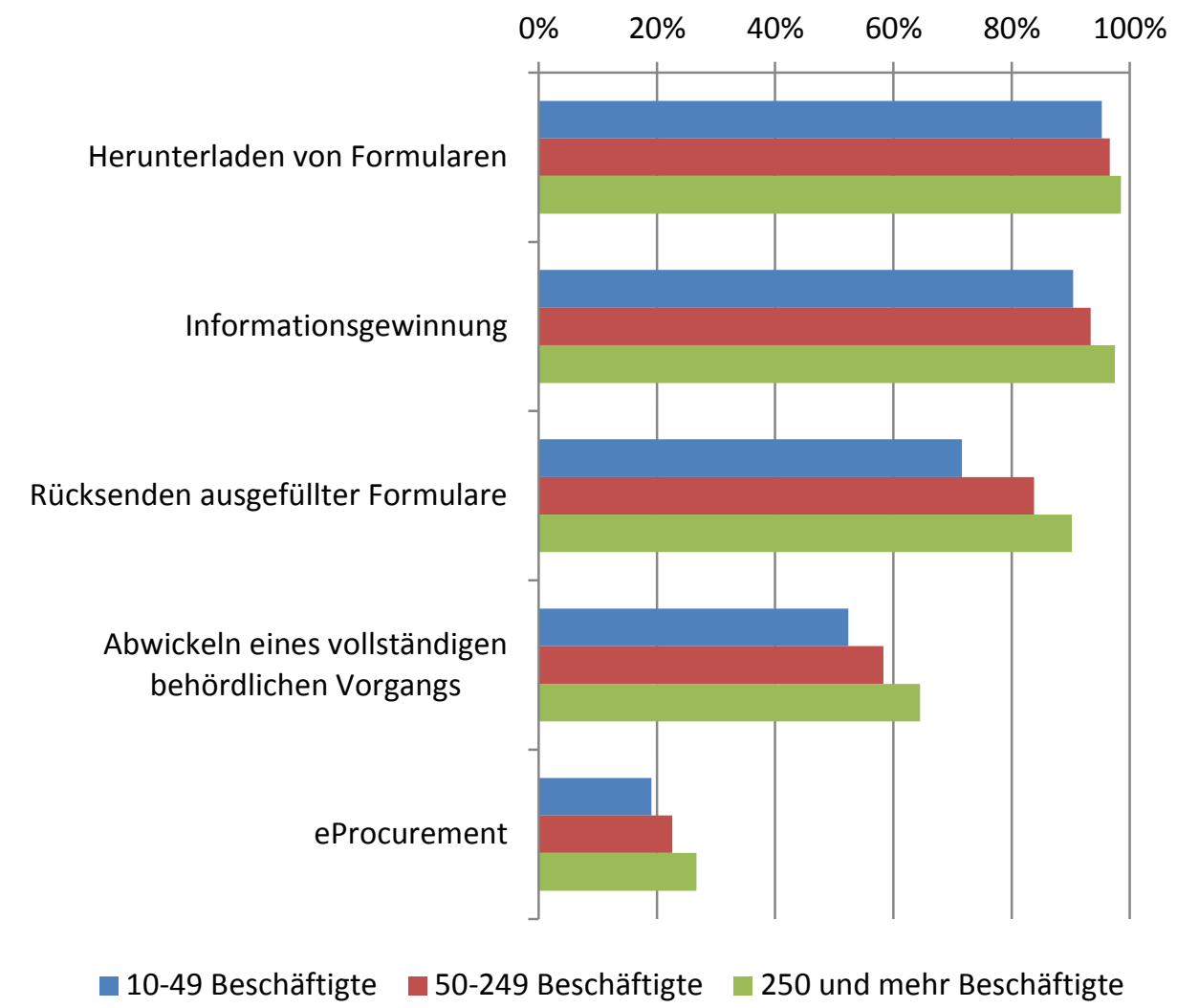

Quelle: Statistik Austria, eigene Darstellung.

Zur Einschätzung des Status der Internetnutzung durch österreichische Unternehmen ist jedoch die Frage des eCommerce von besonderer Relevanz. Dabei lässt sich zwischen Verkäufen und Einkäufen (siehe Abbildung 22 und Abbildung 23) unterscheiden. 
Grundsätzlich sind auch hier größere Unternehmen aktiver als kleinere. Ein Vergleich zwischen Ein- und Verkäufen ist auf Basis der verfügbaren Daten nicht möglich, da erstere den Anteil der Unternehmen und letztere den Umsatzanteil erfassen. Der Umsatzanteil von Verkäufen über das Internet ist über alle Größenklassen hinweg von 2009 auf 2012 gesunken, wobei Unternehmen mit 250 oder mehr Beschäftigten den geringsten Rückgang zu verzeichnen hatten. Dabei bildet der Rückgang für Unternehmen mit 10-49 Beschäftigten insofern eine Ausnahme, als dass hier der Gesamtumsatz leicht gestiegen und der eCommerce-Umsatz gleichzeitig leicht gesunken ist, während für alle anderen Unternehmen ein Wachstum in beiden Kategorien zu verzeichnen war, jedoch der eCommerce nicht mit dem Gesamtwachstum mitzuhalten vermochte.

\section{Abbildung 22 Status - Österreichische Unternehmen mit eCommerce Aktivitäten, Umsatzanteil eCommerce nach Größenklassen, in Prozent aller Unter- nehmen, Vergleich 2009 - 2012}

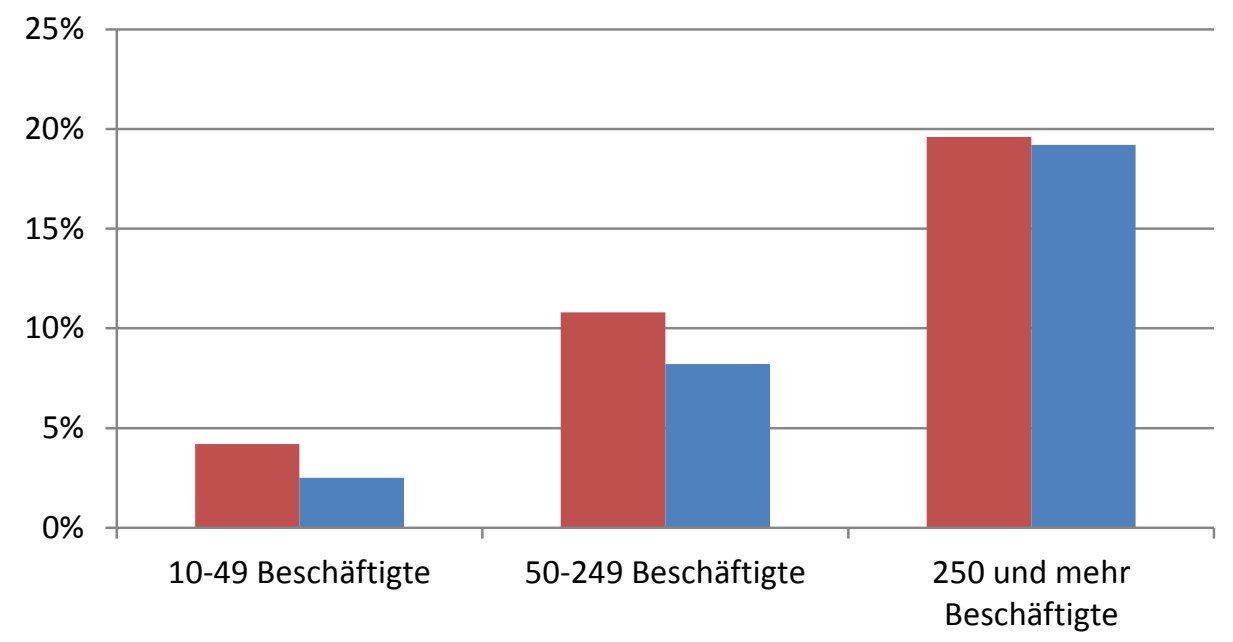

$2009 \quad 2012$

Quelle: Statistik Austria, eigene Darstellung.

Internetnutzung für den Einkauf ist insgesamt wesentlich häufiger als der Verkauf, d.h. 60,4\% aller Unternehmen haben 2012 ihren Einkauf zumindest teilweise über das Internet durchgeführt. Ein weiteres Unterscheidungsmerkmal ist das moderate Wachstum von 2009 auf 2012. 
Abbildung 23 Status - Österreichische Unternehmen mit eCommerce Aktivitäten, Anteil der Unternehmen mit Einkäufen über eCommerce nach Größenklassen, in Prozent aller Unternehmen, Vergleich 2009 - 2012

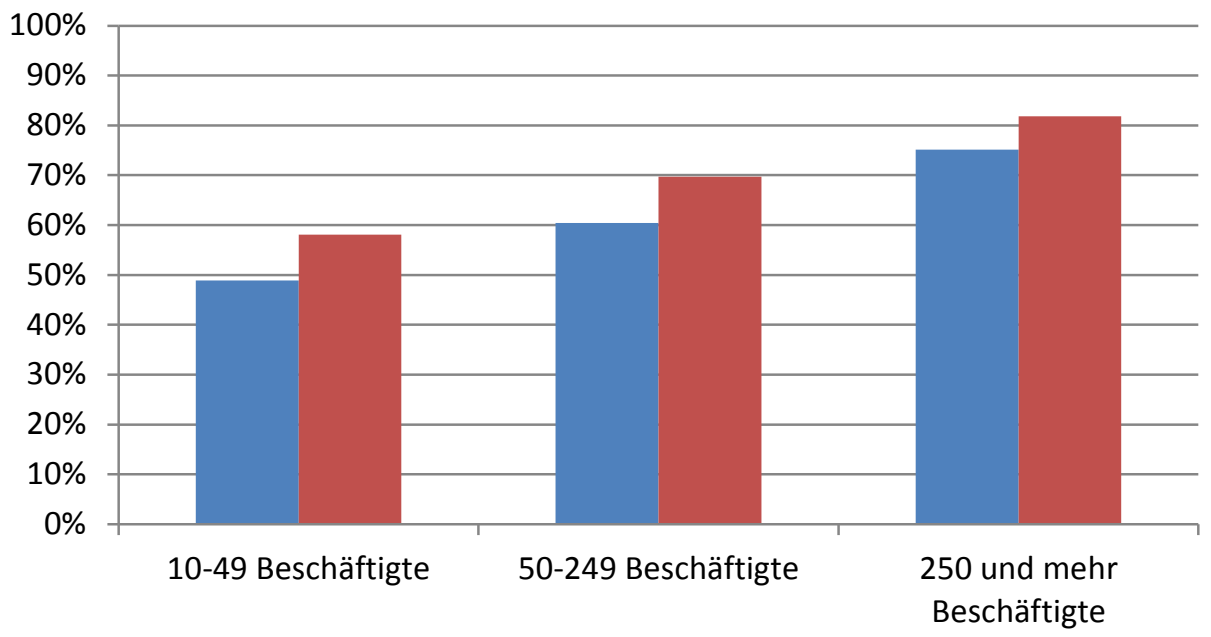

$2009 \square 2012$

Quelle: Statistik Austria, eigene Darstellung.

Laut des 2013 von Statistik Austria veröffentlichten Berichts zur IKT-Nutzung von Unternehmen ${ }^{4}$ nutzen 55\% der österreichischen Unternehmen die Möglichkeit, ihren Beschäftigten Fernzugriff auf das unternehmenseigene E-Mail-System, auf Dokumente oder Software-Applikationen zu geben, wobei dies auf nahezu 100\% der Großunternehmen zutrifft.

\subsection{Internationale Position Österreichs}

Die internationale Position Österreichs, d.h. vor allem innerhalb der EU, gegenüber anderen Staaten ist insgesamt betrachtet etwas weniger positiv. Hinsichtlich der Verfügbarkeit von fixen Breitbandanschlüssen nach Zugangstechnologien (die in aller Regel mit der Schnelligkeit, d.h. der verfügbaren Bandbreite, korrespondiert) liegt Österreich unter dem EU-Durchschnitt was den Anteil schneller Anschlüsse wie DSL und V-DSL betrifft. Allerdings ist dabei zu beachten, dass Österreich im Unterschied zu vielen anderen Staaten eine relativ hohe Verbreitung von mobilen Internetzugängen verfügt. So belegt Österreich bei der Nutzung von mobilem Breitbandinternet den dritten Platz innerhalb der EU (2011).

${ }^{4}$ Statistik Austria, 2013: IKT-Einsatz in Unternehmen. Einsatz von Informations- und Kommunikationstechnologien in Unternehmen 2012, Wien. 
Abbildung 24 Status - Fixe Breitbandanbindung in der EU nach Technologie in Prozent aller Anschlüsse, 2013

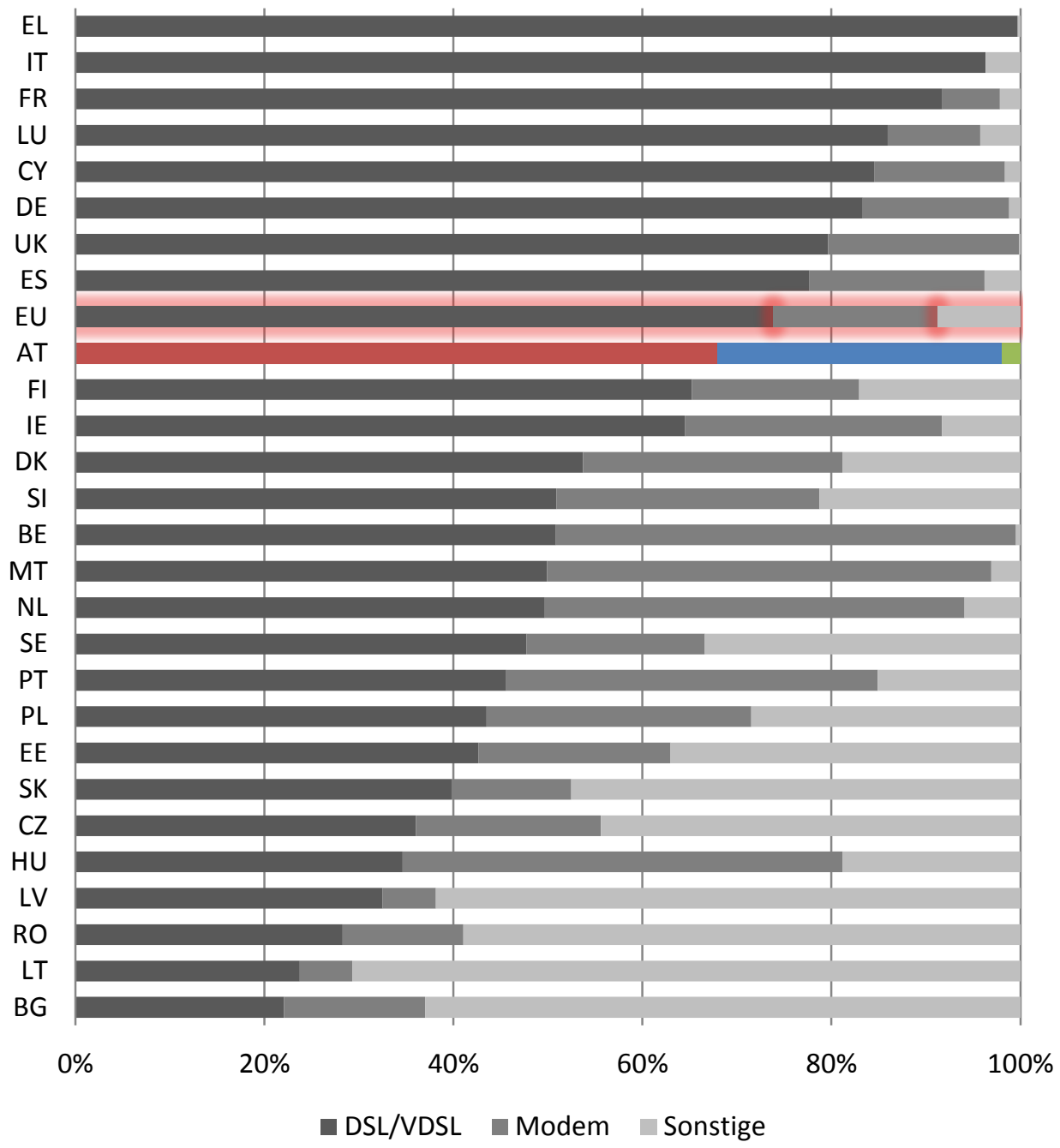

Quelle: Europäische Kommission, eigene Darstellung.

Wichtig in diesem Zusammenhang ist die Verbreitung so genannter next generation access (FTTH, FTTB, VDSL, Cable Docsis 3.0 und andere) Technologien. Hier belegt Österreich nur den fünftletzten Platz mit einer Verbreitung von $11 \%$. Interessant an diesem Vergleich ist die Tatsache, die diesbezüglich führenden Länder zu einem Großteil neue Mitgliedsstaaten sind, deren Infrastruktur überwiegend völlig neu aufgebaut und nicht modernisiert werden musste. 
Abbildung 25 Status - Next Generation Access (FTTH, FTTB, VDSL, Cable Docsis 3.0 und andere) in der EU in Prozent aller fixen Breibandanbindungen, 2013

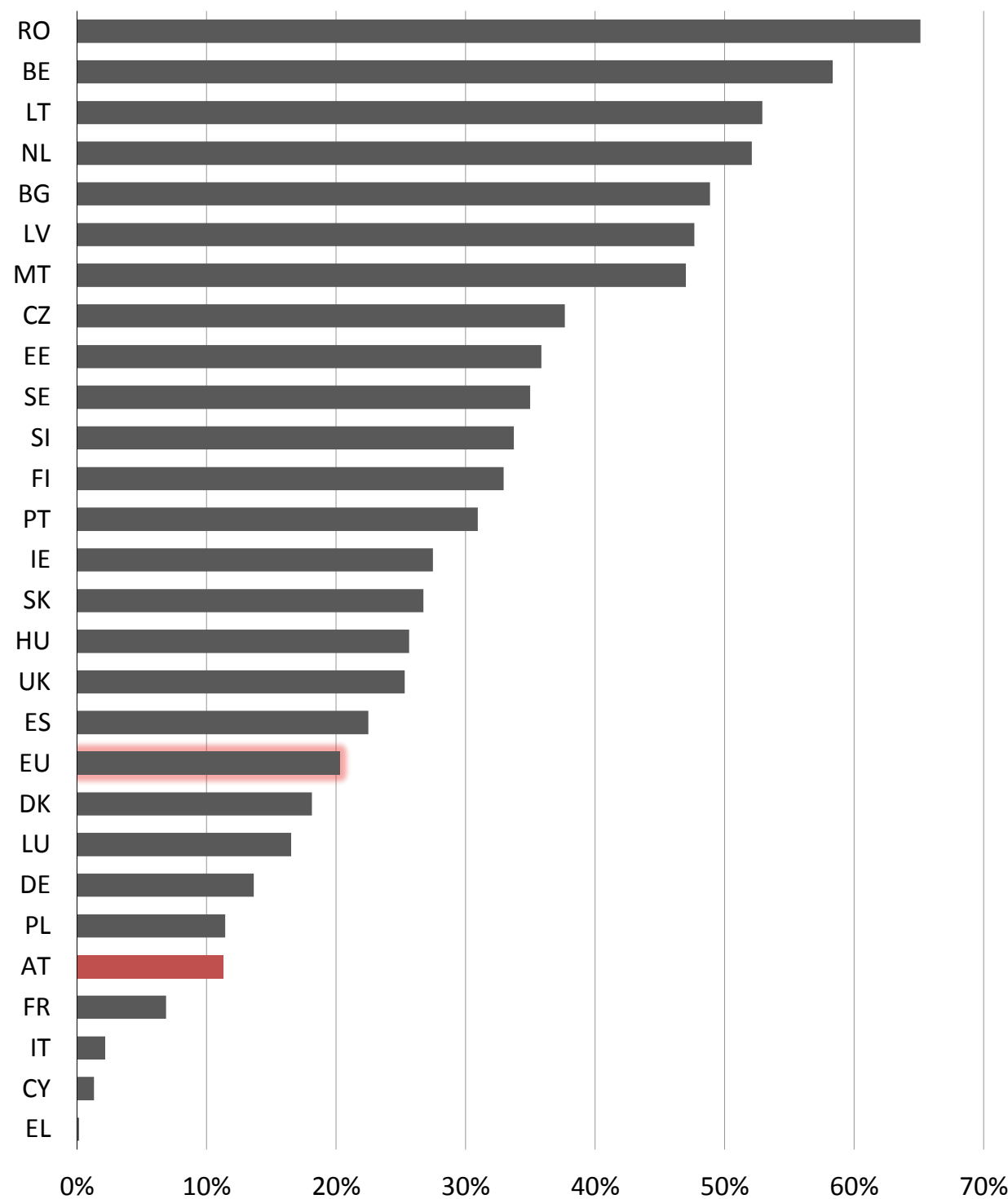

Quelle: Europäische Kommission, eigene Darstellung.

Österreich hat im europäischen Vergleich nicht nur relativ geringe durchschnittliche Bandbreiten, sondern auch einen deutlich geringeren Anteil von Bandbreiten $>10 \mathrm{Mps}$ von $44 \%$ im Vergleich zur EU insgesamt mit 59\%. 
Abbildung 26 Status - Fixe Breitanbindung nach Geschwindigkeit in der EU in Prozent aller Breitbandanbindungen, 2013

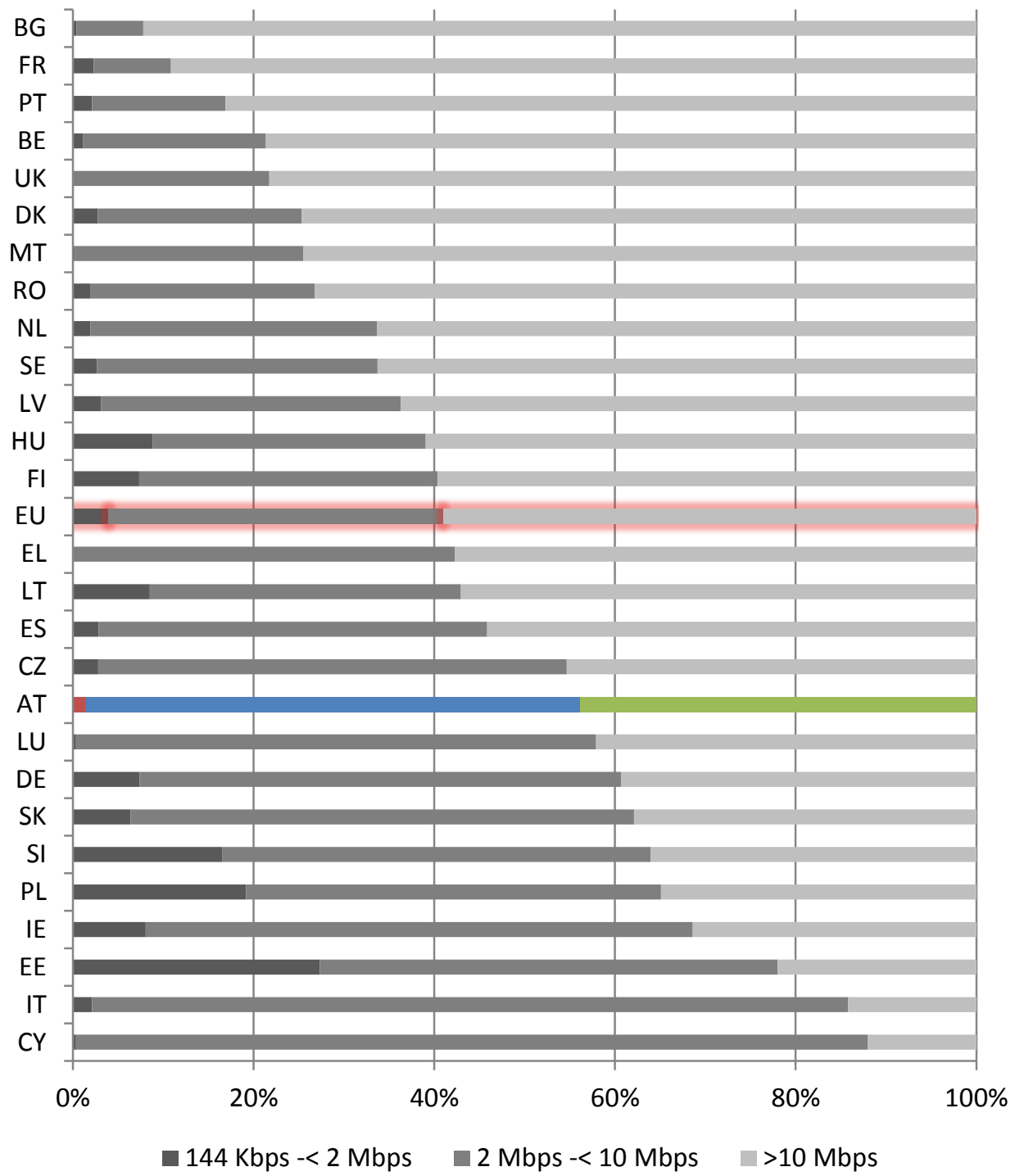

Quelle: Europäische Kommission, eigene Darstellung.

Die Nutzung von Internetanschlüssen für eGovernment ist in Österreich insgesamt, d.h. in allen Unterbereichen, häufiger als im EU-Durchschnitt, wohingegen eCommerce in einigen Bereichen weniger stark und in anderen stärker als im Durchschnitt genutzt wird. Die unternehmerische Nutzung (eBusiness) ist wiederum deutlich wichtiger in Österreich als der EU insgesamt.

Alles in allem lässt sich Österreichs internationale Position als deutlich verbessert gegenüber den Befunden von 2011 bezeichnen. Allerdings bezieht sich diese Verbesserung nicht auf die eigentliche Infrastruktur bzw. auf die besonders zukunftsrelevanten Zugangstechnologien, sondern die Nutzung durch Personen und Unter- 
nehmen. Die operativen Ziele aus dem Bereich Breitbandausbau und -nutzung, die auch für das Förderprogramm AT:net relevant sind, können durchweg als erreicht angesehen werden. Inwieweit die einzelnen Projekte dazu einen Beitrag zu leisten vermochten bzw. inwieweit AT:net als Ganzes ursächlich mit den erreichten Steigerungen zusammenhängt, können nur teilweise beantwortet werden (siehe Kapitel 5.4). Nichtsdestotrotz bedeutet dies einerseits, dass für eine eventuelle Neuauflage andere Ziele (bzw. höhere Ziele, sofern eine Quantifizierung möglich ist) definiert werden müssten und andererseits eine Neuausrichtung auf diejenigen Herausforderungen erfolgen müsste, die aus der vor allem internationalen Vergleichsposition Österreichs abgeleitet werden können, also allen voran die Frage nach den so genannten next generation access Technologien und höheren Bandbreiten als den bereits verfügbaren und als Ziel gesetzten. 


\section{Wirkung der AT:net Förderung}

\subsection{Befragung}

Auf Basis der Daten der Adressdaten der FFG zu den geförderten Organisationen und ihren AT:net Projekten wurde eine Online-Befragung zur Erhebung der Wirkungen auf die Fördernehmer und darüber hinaus durchgeführt. Von den 263 Projektbeteiligungen (entspricht 234 Organisationen) wurden 233 Einladungen zur Teilnahme an der Befragung versandt (26 Organisationen durften aufgrund der Datenschutzbestimmungen nicht angeschrieben werden), d.h. Organisationen mit mehr als einer Beteiligung an AT:net wurden auch zur mehrfachen Beteiligung aufgefordert. Im weiteren Prozess nicht erfolgreicher Kontaktierung und entsprechender Adressrecherchen mussten weitere drei Organisationen aus dem Sample entfernt werden, um den Datenschutz zu entsprechen. Von der Möglichkeit mehrere Fragebögen auszufüllen, machte nur eine Organisation Gebrauch. Im Gegenzug nutzten drei Organisationen die Befragung für mehrere Projekte.

Insgesamt konnten 90 vollständig ausgefüllte Fragebögen zur Auswertung herangezogen werden, was eine Rücklaufquote von 40 \% ergab. Um diese Rücklaufquote zu erreichen, wurden insgesamt fünf Aussendungen zwischen Mitte Dezember 2013 und Mitte Jänner 2014 durchgeführt.

In den folgenden Kapiteln 5.1.1 und 5.1.2 werden die befragten Organisationen/untersuchten Projekte hinsichtlich ihrer wesentlichen Merkmale analysiert und dargestellt. Daran anschließend diskutiert Kapitel 5.2 die Bewertung der Programmimplementierung durch die Fördernehmer und Kapitel 5.4 die verschiedenen Wirkungen und Effekte.

\subsubsection{Befragte Organisationen}

Die abgebildete Verteilung (siehe Abbildung 27) der befragten Organisationen bezüglich ihres zugehörigen Organisationstypen, entspricht etwa dem relativen Anteil der Grundgesamtheit. 


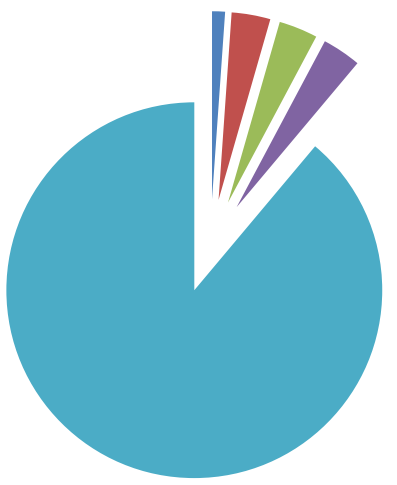

Forschungseinrichtungen $\quad$ Hochschulen
Intermediäre
Unternehmen

$\mathrm{N}=90$

Quelle: KMU Forschung Austria 2014

Die deutlich größte Teilnehmergruppe stellen kommerzielle Unternehmen dar, die etwa 89 \% (absolut 80) der Antworten für sich beanspruchen können. Dieser Prozentsatz liegt leicht über der Gesamtteilnahme von Unternehmen am AT:net - Förderprogramm (84\%). Hochschulen, Intermediäre und Sonstige zeichnen sich jeweils für rd. $3 \%$ (absolut jeweils 3 ) der eingegangenen Antworten verantwortlich. Bei Hochschulen und Intermediäre entspricht diese Verteilung dem Vorkommen in der Grundgesamtheit, während die Sonstigen eine etwas geringere Rücklaufquote aufweisen (Grundgesamtheit $8 \%$ ). Schließlich sind die Forschungseinrichtungen durch einen Befragungsteilnehmer ebenfalls vertreten (1\%), wobei sie auch in Summe bei den bewilligten Förderprojekten den relativ (2\%) und absolut (5) geringsten Anteil der Fördernehmer darstellen.

Die Differenzierung der befragten Unternehmen hinsichtlich ihrer MitarbeitergröBenklasse (siehe Abbildung 28) zeigt ein deutliches Übergewicht zu Gunsten der Kleinst- und Kleinunternehmen, welche in Summe rund 90\% der untersuchten Fördernehmer bilden. 


\section{Abbildung 28 Befragte Unternehmen nach Größenklassen}

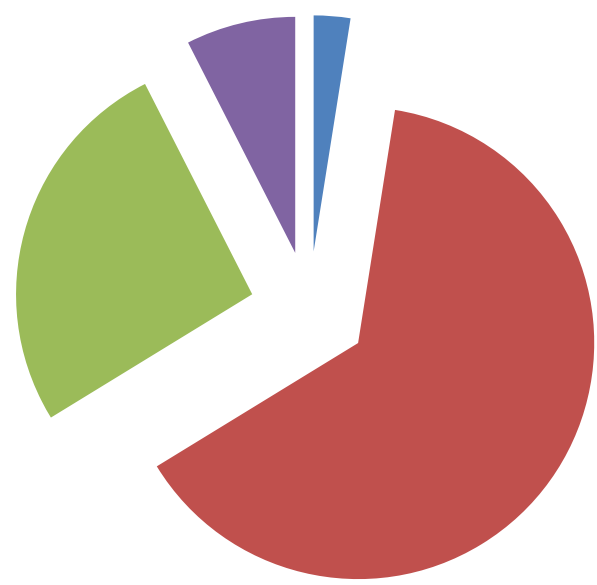

EPU Kleinstunternehmen Kleinunternehmen $\square$ Mittlere Unternehmen

$\mathrm{N}=80$

Quelle: KMU Forschung Austria 2014

Nahezu zwei Drittel aller Unternehmen (64\%) beschäftigen zwischen 1 und 10 Mitarbeiter, wobei durchschnittlich knapp vier Beschäftigte angestellt sind. Kleinunternehmen stellen etwa ein Viertel $(26 \%)$ der Befragten und beschäftigen im Mittel 23 Mitarbeiter. 8\% der Unternehmensantworten entfallen auf Betriebe mittlerer Größe, wobei die Beschäftigtenzahlen zwischen 51 und 240 Mitarbeitern schwanken. Die kleinste Gruppe wird von den Ein-Personen-Unternehmen gebildet und umfasst rd. $3 \%$ der befragten Unternehmen.

\subsubsection{Projekte}

Mehr als zwei Drittel (69\%) der untersuchten AT:net Projekte konnten bereits abgeschlossen werden (absolut 62), dementsprechend sind etwa 31\% der geförderten Breitbanddienstvorhaben noch in ihrer Durchführungsphase (absolut 28 Projekte). 
Abbildung 29 Untersuchte Projekte: abgeschlossene vs. laufend

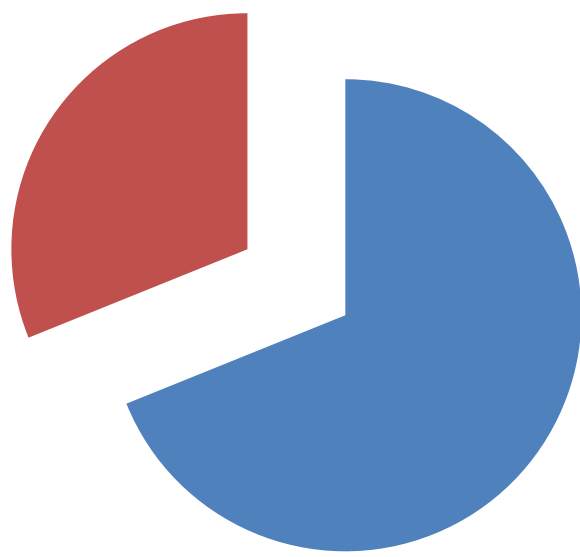

abgeschlossen $\square$ laufend

$\mathrm{N}=90$

Quelle: KMU Forschung Austria 2014

Betrachtet man den Projektstatus (siehe Abbildung 30) in Hinblick auf die unterschiedlichen Projekttypen so zeigt sich, dass der überwiegende Anteil der abgeschlossenen Förderprojekte nicht im Bereich der Infrastrukturvorhaben angesiedelt war. Lediglich 18 (rd. 30\%) der 61 entlasteten Verwertungsvorhaben dienten dem Ausbau bzw. der Errichtung von Infrastrukturen im Bereich der Breitbanddienste. Dem gegenüber stehen die laufenden Projekte, welche hingegen 57\% der Befragten Fördergelder im Bereich der IT-Infrastruktur in Anspruch genommen (absolut 16). 


\section{Abbildung 30 Untersuchte Projekte: Infrastrukturprojekte vs. andere}

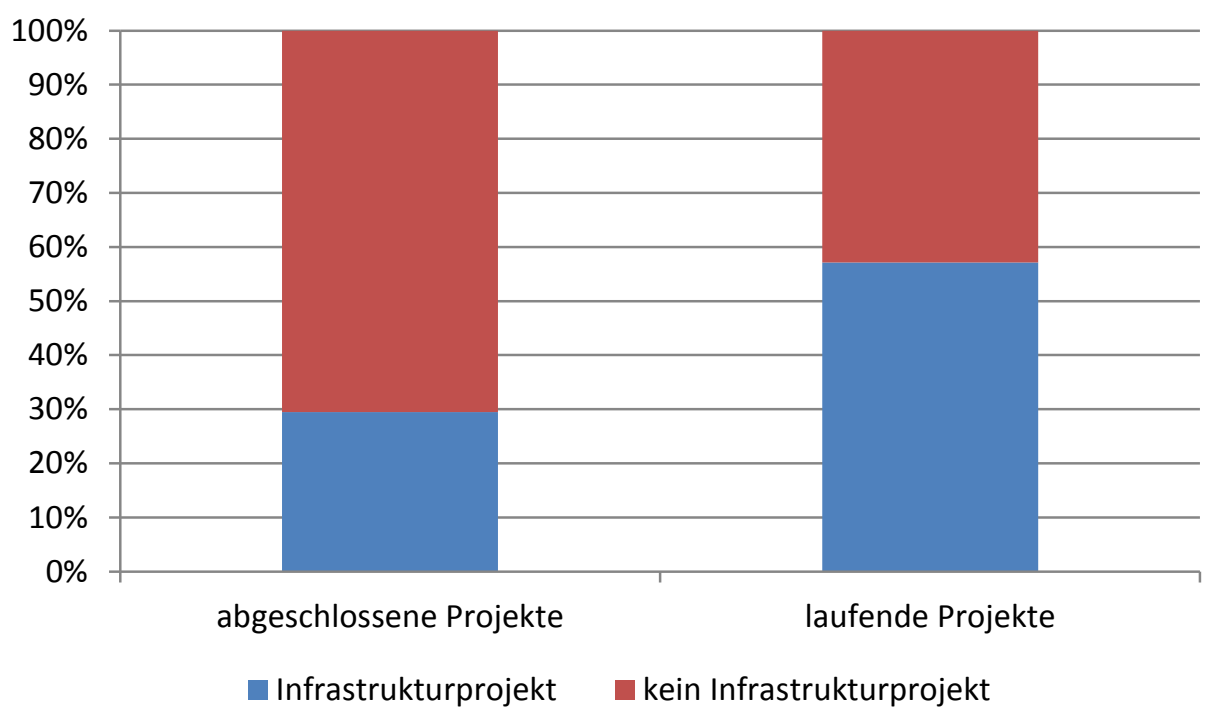

n = 61 (abgeschlossene Projekte) bzw. 28 (laufende Projekte)

Quelle: KMU Forschung Austria 2014

Ähnlich ist hingegen der Anteil jener Fördernehmer, deren AT:net Projekt die Weiterführung eines öffentlich geförderten F\&E-Projektes bedeutet. Sowohl bei den abgeschlossenen als auch bei den laufenden Projekten liegt inr Anteil im niedrigen, zweistelligen Bereich: 15\% der abgeschlossenen und 12\% der derzeit laufenden Projekte.

In Hinblick auf das primäre Ziel der geförderten Projekte lässt sich zwischen dem Erschließen neuer Aktivitäten (etwa Geschäfts- oder Forschungsthemen) und der Erweiterung bestehender Aktivitäten unterscheiden, wobei durchaus auch beide Ziele gemeinsam im Zentrum eines Verwertungsvorhabens stehen können (siehe Abbildung 31 . 


\section{Abbildung 31 Primäres Ziel der untersuchten Projekte}

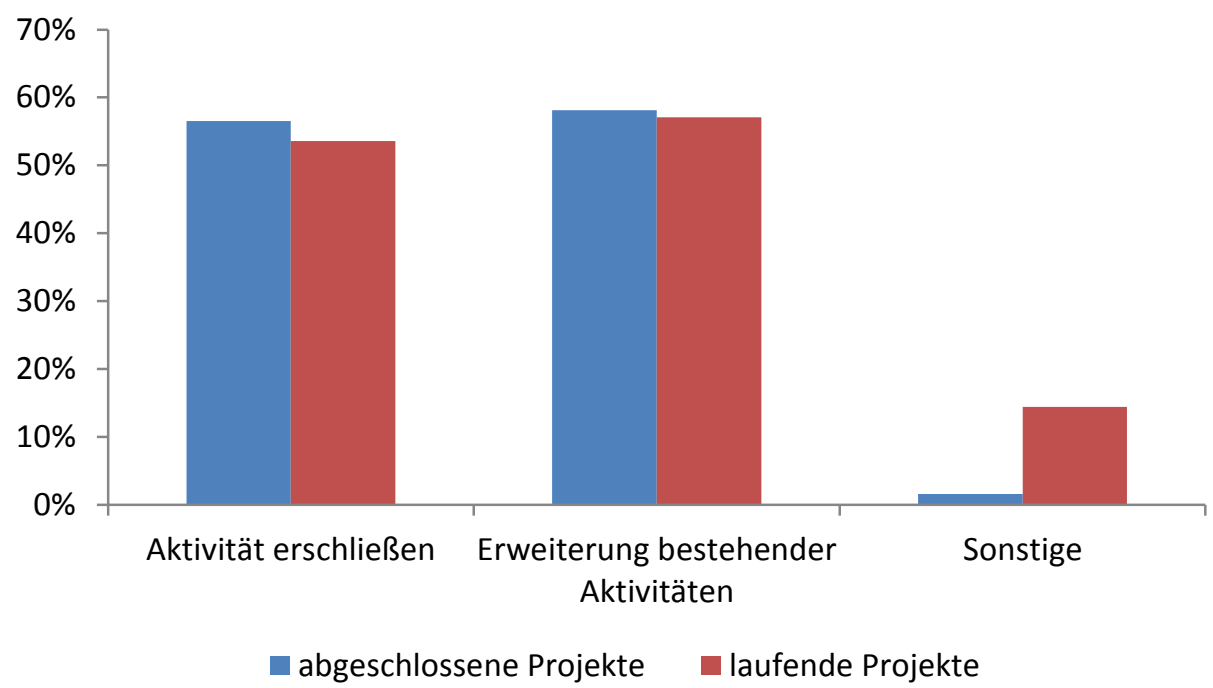

n = 61 (abgeschlossene Projekte) bzw. 26 (laufende Projekte)

Quelle: KMU Forschung Austria 2014

Sowohl abgeschlossene als auch laufende Projekte weisen diesbezüglich Werte zwischen $50 \%$ und $60 \%$ auf. Die höchsten erzielten Werte weisen die abgeschlossenen bzw. laufenden Förderprojekte in Zusammenhang mit der Erweiterung von bestehenden Aktivitäten auf (58\% bzw. 57\%). Organisationen die AT:net Projekte nicht im Zuge der Aktivitätserweiterung- bzw. Erschließung durchgeführt haben, hatten in der Kategorie „Sonstige“ die Möglichkeit ein anderes Ziel anzugeben bzw. den spezifischen Projektnutzen zu beschreiben. Davon machten fünf Unternehmen Gebrauch, wobei die zugehörigen, spezifischen Antworten untenstehend angeführt sind:

- Neues Projekt

- Ausbau und Weiterentwicklung

- Erweiterung der Produktpalette

- Breitband im ländlichen Raum

- Datensicherheit

- Ermöglichung alle Studierendenheime in Graz mit kostengünstigem Internet- und Intranetzugang zu versorgen

Etwa ein Drittel der befragten Organisationen haben, abgesehen von ihren AT:net Beteiligungen, keine Erfahrung mit Förderprogrammen (siehe Abbildung 32). Die $68 \%$ mit Erfahrung außerhalb von AT:net nutz(t)en mehrheitlich nationale Förderprogramme (47\%) gefolgt von regionalen (33\%) und internationalen $(23 \%)$. 
Abbildung 32 Erfahrung der befragten Organisationen mit anderen Fördermaßnahmen

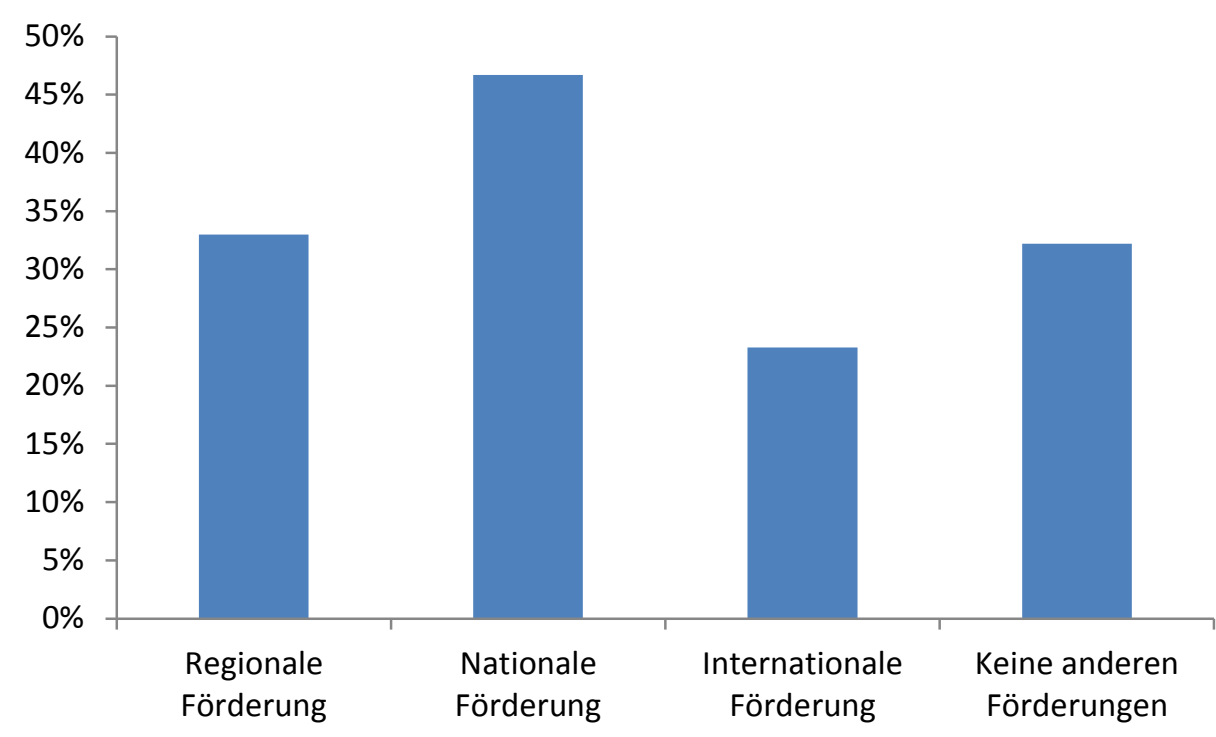

$\mathrm{n}=89$

Quelle: KMU Forschung Austria 2014

Hinsichtlich nationaler Fördermaßnahmen sind vor allem FFG-Förderprogramme und aws Maßnahmen von Relevanz, während international vor allem die EU Rahmenprogramme von den AT:net Fördernehmern genutzt werden/wurden (siehe Tabelle 1)

Tabelle 1 Erfahrung der befragten Organisationen mit nationalen und EU Fördermaßnahmen

\begin{tabular}{|l|l|l|l|}
\hline & & Anzahl & $\%$ \\
\hline National & FFG & 36 & $40 \%$ \\
\hline & AWS & 15 & $17 \%$ \\
\hline International & EU FP & 10 & $11 \%$ \\
\hline $\begin{array}{l}\text { Andere EU Förderun- } \\
\text { gen }\end{array}$ & 9 & $10 \%$ \\
\hline
\end{tabular}

$\mathrm{n}=40$ (national) bzw. 17 (international)

Quelle: KMU Forschung Austria 2014

Beispiele für andere Fördermaßnahmen, die von den Befragten abgesehen von AT:net genutzt wurden, umfassen die Folgenden:

- $\mathrm{AMS} / \mathrm{ESF}$ 
- Regio13

- Clusterkooperationsprogramme

- Departure

- Österreichisches Filminstitut

- InnovationsassistentInnen/-beraterInnen

- Unternehmensbeteiligungsgesellschaft mbH Oberösterreich

- Wiener Filmfonds

- ZIT

- ATLANTIS (EU/US Cooperation Programme in Higher Education and Vocational Training)

- EU Interreg IV

- Kärntner Wirtschaftsförderungs Fonds

- Golnternational

Diese vielfach aus eher wirtschaftspolitischen Förderungen bestehende Auflistung sowie die vorangegangenen Analysen verdeutlichen erneut die heterogene $\mathrm{Zu}-$ sammensetzung der Fördernehmer des Programms, aus einerseits Organisationen mit starker Forschungs-, Entwicklungs- und Innovationsintensität und der entsprechenden Kenntnis verfügbarer Unterstützungsleistungen und andererseits aus Organisationen (vor allem Unternehmen und Intermediäre), die als wenig innovationsorientiert zu kennzeichnen sind (siehe auch Kapitel 3 bezüglich der Analyse der Nutzung von FFG Programmen abgesehen von AT:net). Verstärkt wird dieser Eindruck noch dadurch, dass lediglich ein geringer Anteil der untersuchten Projekte von den befragten Organisationen als explizite Weiterführung eines geförderten Forschungsprojekts klassifizieren: für $15 \%$ der abgeschlossenen und $11 \%$ der laufenden Projekte wurde die entsprechende Frage bejaht. Dieses Ergebnis kann allerdings nicht überbewertet werden, da interne nicht-geförderte Entwicklungsprojekte in den geförderten Anwendungsfeldern von relativ großer Bedeutung sind. Unter der Annahme, dass vor allem KMU die Durchführung von komplexeren, anspruchsvolleren Projekten nur selten ohne finanzielle Unterstützung gelingt, weist es aber nichtsdestotrotz auf einen relativ geringen Bezug (der Projekte und nicht zwangsläufig der Organisationen selbst) zu Forschung hin. 


\subsection{Projekt-/Programmimplementierung}

Die folgende Abbildung 33 zeigt die Bewertung verschiedener Implementierungsaspekte des Förderprogramms. Insgesamt wird deutlich, dass diese überwiegend positiv bewertet werden, was zum einen mit der inherenten Niedrigschwelligkeit des Instruments der de-minimis zusammenhängt, andererseits aber nicht unterbewertet werden sollte, da in aller Regel insbesondere KMU die Unverhältnismäßigkeit von vor allem administrativen Hürden bei der Beantragung und Abwicklung von Förderungen kritisieren. 
Abbildung 33 Bewertung der Programmimplementierung durch die befragten Fördernehmer

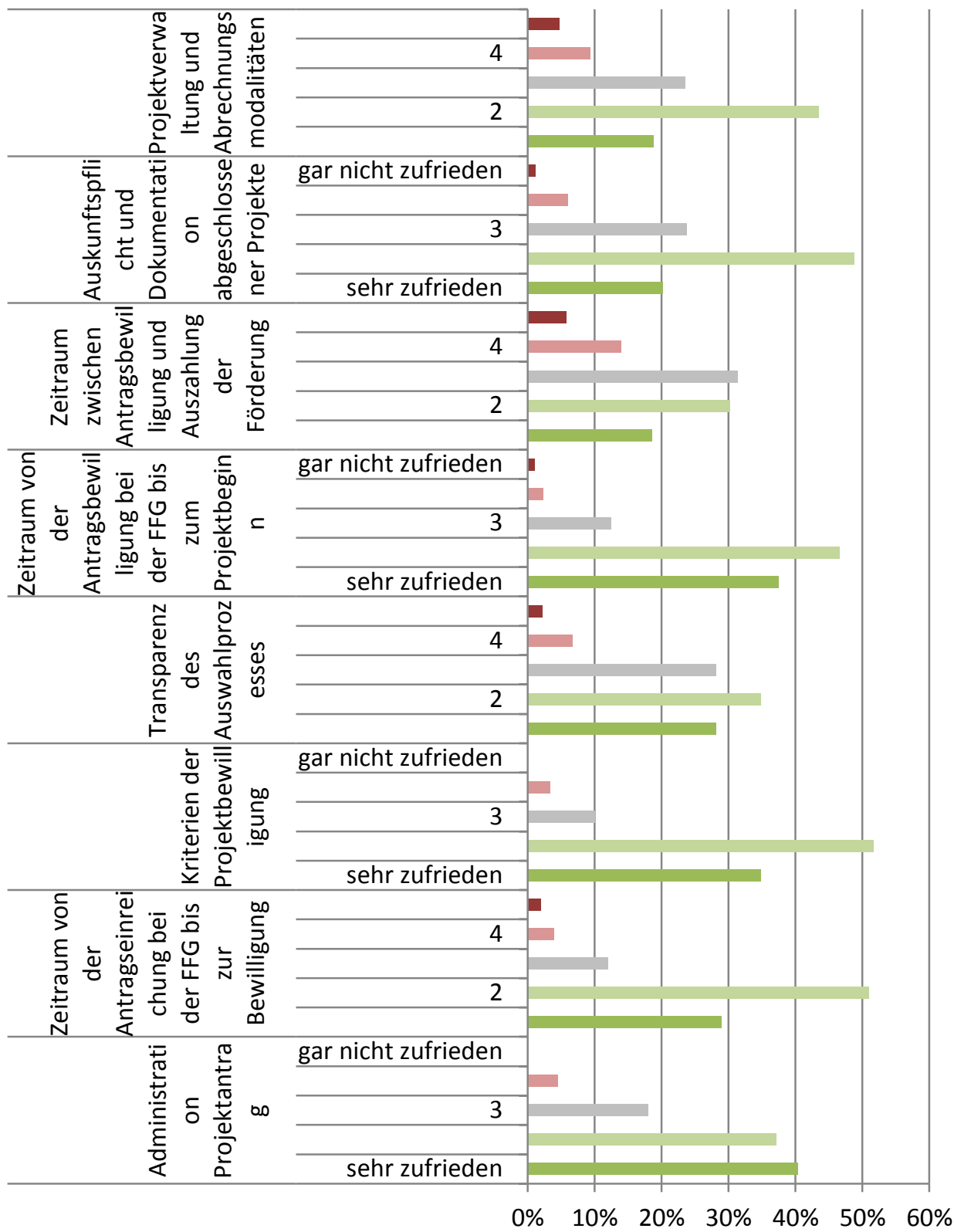

$\mathrm{n}=89$

Quelle: KMU Forschung Austria 2014

Die höchste Zufriedenheit der Fördernehmer erreicht AT:net hinsichtlich der Kriterien der Projektbewilligung (87\% der Fördernehmer sind hier zufrieden oder sehr zufrieden), gefolgt vom Zeitraum von der Antragsbewilligung bei der FFG bis zum Projektbeginn (84\%) und dem Zeitraum von der Antragseinreichung bei der FFG bis zur Bewilligung (80\%). Obwohl nach wie vor deutlich positiv bewertet, schnei- 
den die folgenden Aspekte am schlechtesten ab: Zeitraum zwischen Antragsbewilligung und Auszahlung der Förderung (49\%), Projektverwaltung und Abrechnungsmodalitäten (62\%) sowie die Transparenz des Auswahlprozesses (63\%).

\subsection{Additionalität}

Die Frage nach der Additionalität der in AT:net geförderten Projekte kann unter zwei verschiedenen Aspekten betrachtet werden: (1) ob die Projekte auch ohne die entsprechende Unterstützung durchgeführt worden wären und (2) ob es infolge der Projekte zu direkt zuordenbaren zusätzlichen Investitionen gekommen ist.

Zunächst ist festzuhalten, dass es laut Selbsteinschätzung der befragten Fördernehmer nur sehr geringe Mitnahmeeffekte (d.h. Projekte, die auch ohne Förderung unverändert durchgeführt worden wären) gegeben hat (siehe Abbildung 34). Der überwiegende Teil der Projekte hätte ohne die Förderung in deutlich verringertem Ausmaß oder mit deutlicher zeitlicher Verzögerung durchgeführt werden müssen.

\section{Abbildung 34 Additionalität der untersuchten Projekte}

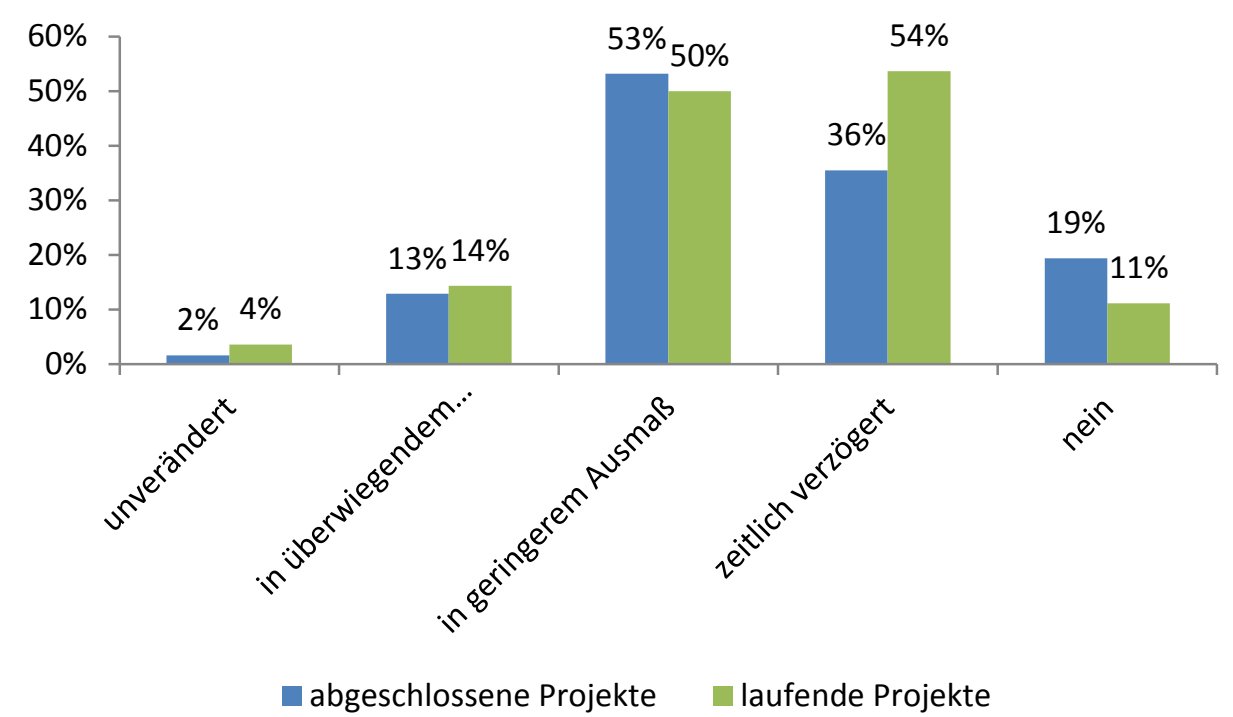

$\mathrm{n}=62$ (abgeschlossen) bzw. 27 (laufend)

Mehrfachantworten möglich

Quelle: KMU Forschung Austria 2014

Volle Additionalität, d.h. eine Nicht-Durchführung ohne die AT:net Förderung, ist für 19 bzw. $11 \%$ der Projekte gegeben. Insgesamt ist die Frage nach der Bedeutung der Förderung für die Durchführung nicht eindeutig zu beantworten. Der Anteil der Projekte, die anderenfalls überhaupt nicht durchgeführt worden wären, ist einerseits gering, andererseits vor dem Hintergrund der relativen Marktnähe der Projek- 
te wiederum nicht zu vernachlässigen. Für die abgeschlossenen Projekte ist auf Basis der Befragungsergebnisse davon auszugehen, dass bis zu einem Fünftel von einer Vermarktung in der zur Förderung eingereichten Form abgesehen hätten, obwohl kaum technologisches Risiko gegeben war. Wenn es also Faktoren gibt, die verhindern können, dass vor allem Unternehmen den letzten entscheidenden Schritt zur Kapitalisierung ihrer Innovationsprojekte machen und AT:net in diesen Fällen den Ausschlag geben konnte, dann sind die erwähnten Ergebnisse absolut als Nachweis der Hebelwirkung des Programms geeignet (auch wenn über den wirtschaftlichen Erfolg keine Aussagen gemacht werden können). Verstärkt wird dieser Eindruck, bedenkt man, welche Auswirkungen zeitliche Verschiebung oder Limitierung von Aktivitäten auf den wirtschaftlichen Erfolg vor allem in einem dynamischen Anwendungsfeld wie IKT haben können.

Von den 62 untersuchten abgeschlossenen Projekten haben nur 24 angegeben, dass sie zusätzliche (Folge-) Investitionen im Zusammenhang mit ihrem AT:net Projekt getätigt haben. Diese Folgeinvestitionen sind im Sinne der InputAdditionalität eine zusätzliche Bewertungsdimension des Förderprogramms. Im Vergleich zu eher klassischen Forschungs- oder Innovationsförderungsprogrammen ist AT:net insofern auch bezüglich der Input-Additionalität ein Sonderfall, da es mit der Unterstützung der Marktüberleitung bereits eine Phase des Innovationsprozesses unterstützt wird, in dem üblicherweise Investitionen zusätzlich zu einem Projekt notwendig sind. Während also 40\% Fördernehmer mit Folgeinvestitionen in anderen Programmen eine möglicherweise eine eher geringe Quote darstellen, kann das im Rahmen von AT:net durchaus als positives Ergebnis bezeichnet werden. Die folgende Abbildung 35 verdeutlicht darüber hinaus, in welchen Bereichen die Folgeinvestitionen getätigt wurden. 
Abbildung 35 Input-Additionalität (Folgeinvestitionen) der abgeschlossenen Projekte

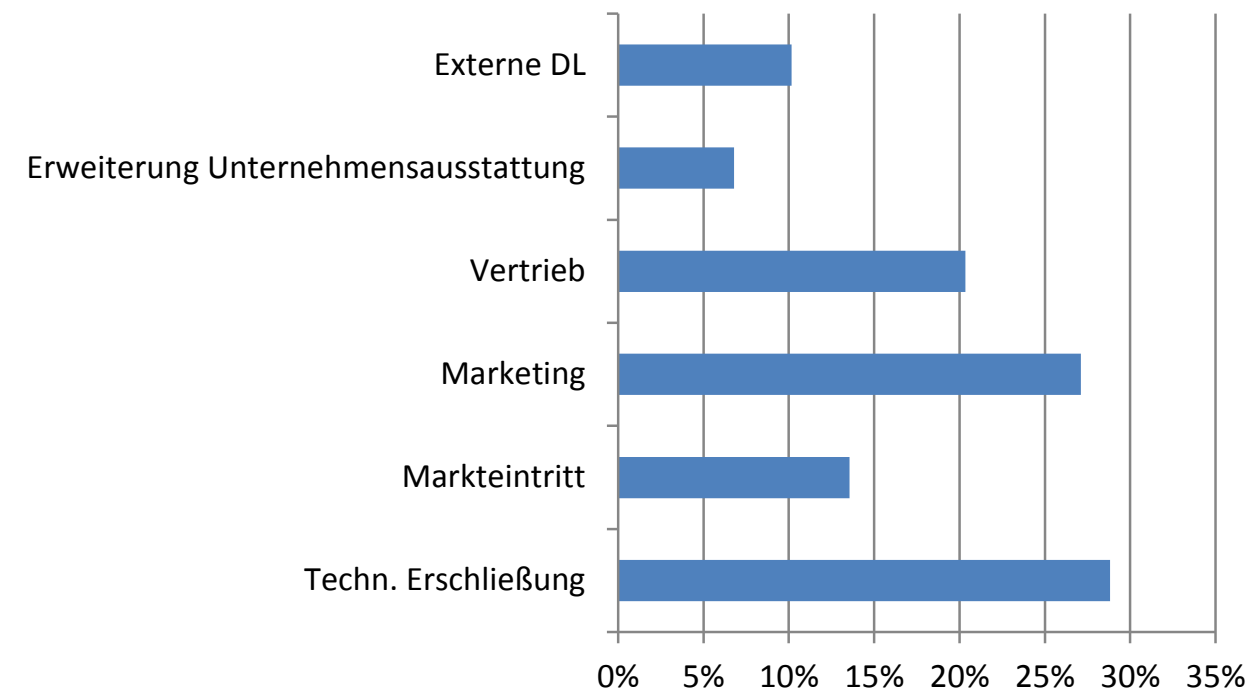

$\mathrm{N}=24$

Mehrfachantworten möglich.

Quelle: KMU Forschung Austria 2014

Unter den Organisationen mit Folgeinvestitionen sind vor allem zusätzliche Ausgaben für die weitere technische Erschließung, d.h. Investitionen in technologische Aspekte, von Bedeutung sowie Ausgaben für Marketing und Vertrieb. Die Bandbreite der absoluten zusätzlichen Investitionen reicht dabei von 2.000 bis 3.000.000 Euro. Dies verdeutlicht, dass Folgeinvestitionen nicht nur eher eine Ausnahme für AT:net Projekte sind, sondern auch eher nur punktuell von kritischer Masse sind. Einschränkend gilt allerdings, dass mit dem AT:net Projekt ja in aller Regel die notwendigen Investitionen in eine Vermarktung bereits getätigt worden sind.

\subsection{Projektergebnisse und -wirkungen}

\subsubsection{Ergebnisse}

Betrachtet man die abgeschlossenen Projekte in Hinblick auf die erfolgreiche Umsetzung aus technischer und wirtschaftlicher Sicht, so lässt sich erkennen, dass $100 \%$ der Antwortenden die technische Umsetzung erfolgreich abschließen konnten. Aus wirtschaftlicher Betrachtungsweise sind mehr als drei Viertel $(76 \%)$ der beendeten Förderprojekte erfolgreich verlaufen (siehe Abbildung 36). 
Abbildung 36 Zielerreichung der abgeschlossenen Projekte aus technischer und wirtschaftlicher Sicht

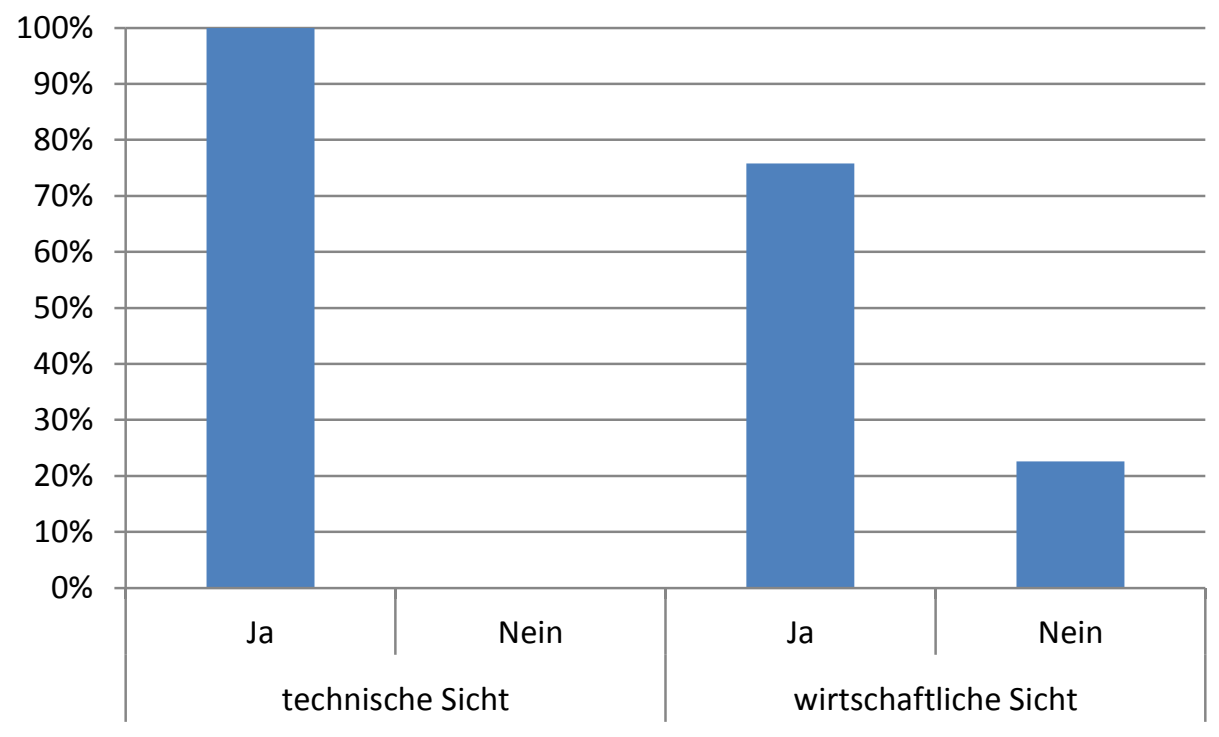

$\mathrm{n}=61$

Quelle: KMU Forschung Austria 2014

Die untersuchten Infrastrukturprojekte schneiden hinsichtlich der Erreichung der ökonomischen Ziele leicht besser ab (83\% gegenüber $74 \%$ ). Was die überwiegend positive Bewertung der Zielerreichung durch die Fördernehmer jedoch deutlich abschwächt ist die Tatsache, dass Unternehmen die Erreichung insgesamt deutlich kritischer bewerten: nur etwa jedes zweite Unternehmen sieht die wirtschaftlichen Ziele als bereits erreicht an.

Einige der befragten Unternehmen nutzten die Möglichkeit, ihre spezifischen Gründe anzugeben, die eine wirtschaftliche Verwertung bislang verhindert haben:

- $\quad$ zu wenig Kunden, wenig Marketing (kein Budget)

- $\quad$ wirtschaftliche Situation, Monopolistischer Markt (Infrastruktur)

- Verzögerung bei der Entwicklung - Vermarktung hat später als geplant begonnen. Erste Kunden konnten aber bereits gewonnen werden

- technologische Entwicklung hat länger als geplant gedauert

- technische Entwicklung von Alternativen

- Schwächen im Vertrieb

- schwache Projektpartner im Verkauf im Ausland

- Marketingkonzept ist noch nicht ausreichend umgesetzt 
- mangelnde Weiterfinanzierung, zu geringer Ausbau des Breitbandnetzes

- Konkurrenz durch neue Anbieter/Technologien

- keine Kundenakzeptanz

- Entwicklung dauert länger

Zusammengefasst lassen diese Gründe erkennen, dass vor allem Verzögerungen in der technologischen Entwicklung zu Verzögerungen im Vermarktungserfolg geführt haben. Andererseits ist eine wesentliche Ursache für die bisher ausgebliebenen ökonomischen Erfolge die fehlende Nachfrage, entweder aufgrund existierender Konkurrenz/konkurrierender bzw. alternativer Technologien/fehlenden Kundeninteresses, was als mangelnde Kenntnis der Markt- und Konkurrenzsituation interpretiert werden kann. Hinzu kommen jene Fälle, in denen die Vermarktung aufgrund mangelnden Ressourceneinsatzes bislang nicht erfolgreich abgeschlossen werden konnte.

Die Verwertung der Projektergebnisse (siehe Abbildung 37) durch die geförderten Organisationen zeigt zunächst deutlich, das AT:net in erster Linie die unmittelbare Vermarktung im Sinne neuer Dienstleistungen und Produkte unterstützen soll, wobei Dienstleistungen noch deutlich häufiger Ergebnis der Projektverwertung sind. Auffällig ist zusätzlich die relativ große Bedeutung von Vermarktungs/Marketing-relevanten Neuerungen bereits vorhandener Produkte oder Dienstleistungen (z.B. Preismodelle oder Vertriebskanäle). Andere Verwertungsformen wie neue Prozess, Designinnovationen oder anschließende Forschungs-, Innovationsund Entwicklungsaufträge sind von deutlich nachrangiger Bedeutung. Interessanterweise zielen laufende Projekte in ihrer Gesamtheit auf eine weniger fokussierte Verwertungsstrategie ab, was jedoch als überwiegend projektspezifisch angenommen werden muss und nicht als Hinweis auf eine von der ursprünglichen Planung abweichende tatsächliche Verwertung.

Betrachtet man nur jene Organisationen, die ihre wirtschaftlichen Zielsetzungen als erreicht betrachten, zeige sich keine signifikanten Unterschiede bis auf den leicht höheren Anteil von neuen/verbesserten Dienstleistungen als Verwertung der Projektergebnisse. 


\section{Abbildung 37 Verwertung der Projektergebnisse}

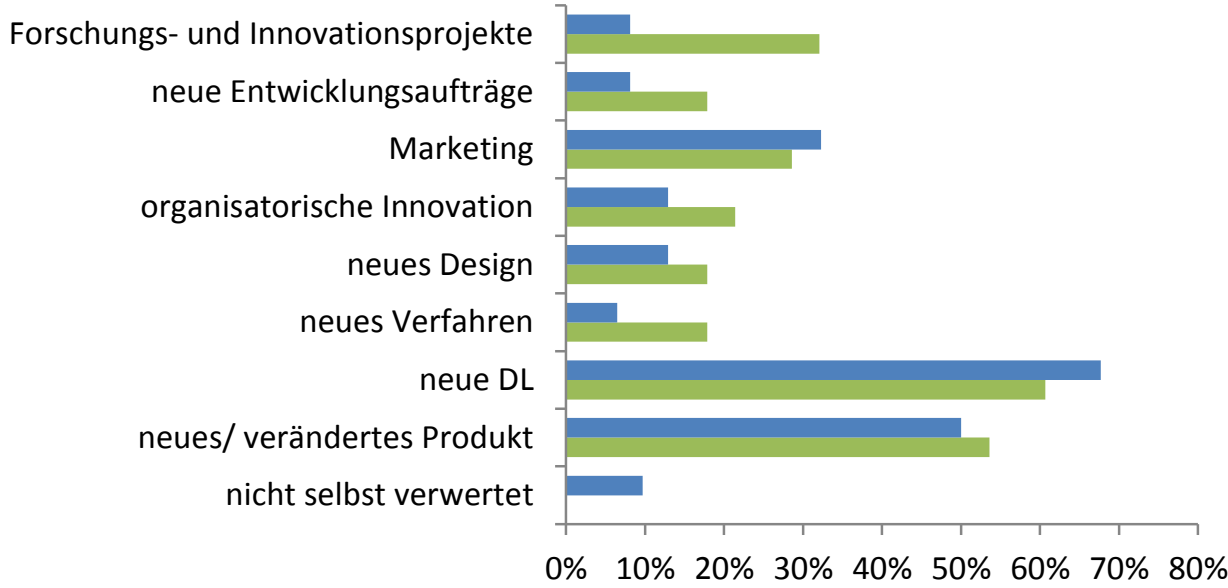

$\square$ abgeschlossene Projekte $\square$ laufende Projekte

$\mathrm{N}=62$ (abgeschlossen) bzw. 27 (laufend)

Mehrfachantworten möglich

Tatsächliche Verwertung für abgeschlossene Projekte, geplante Verwertung für laufende Projekte

Quelle: KMU Forschung Austria 2014

Daran anschließend ist die Frage der Nachhaltigkeit von Bedeutung (siehe Abbildung 38): mehr als drei Viertel der Befragten gaben an, dass das Projektergebnis in adaptierter bzw. weiterentwickelter Form nach wie vor Teil des Angebotsportfolios ist und für weitere $13 \%$ ist das Projektergebnis sogar in unveränderter Form Portfoliobestandteil. 
Abbildung 38 Projektergebnis als Teil des Angebotsportfolios der geförderten Organisationen (abgeschlossene Projekte)

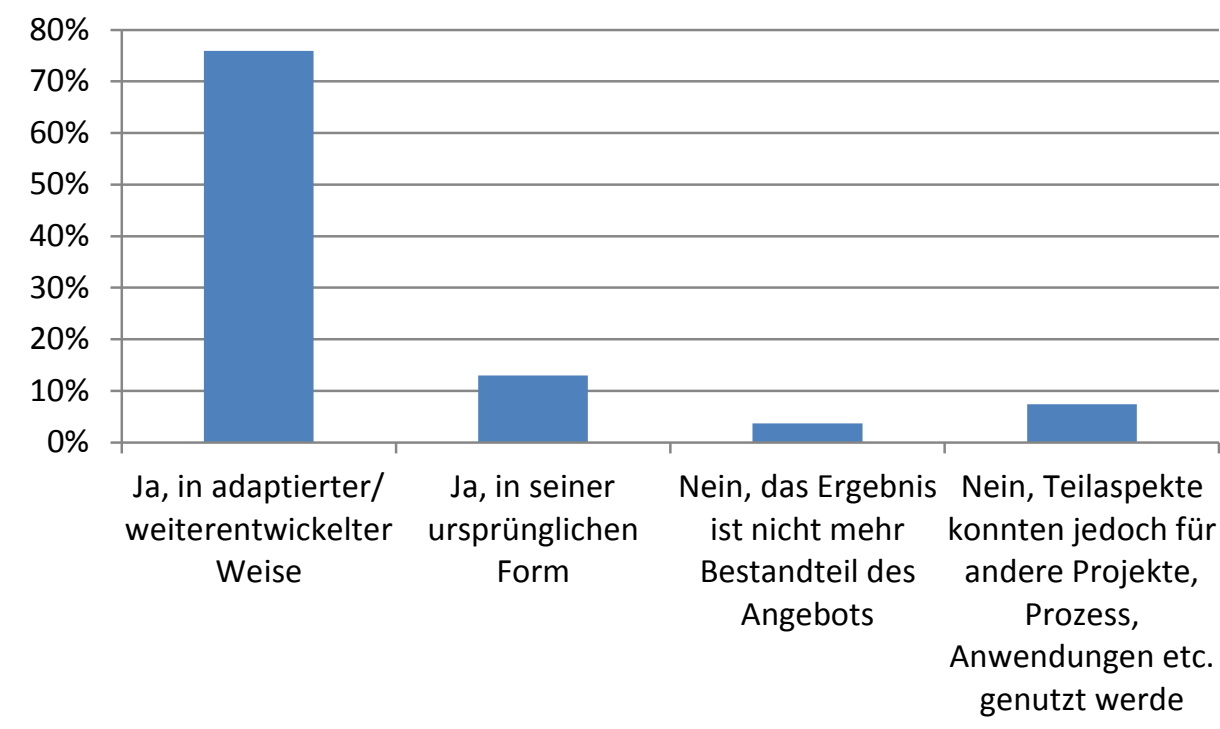

$\mathrm{n}=54$

Quelle: KMU Forschung Austria 2014

Weitere 7\% konnten Elemente für andere Projekte etc. nutzen, was in Summe bedeutet, dass insgesamt $96 \%$ der befragten Organisation mit abgeschlossenen Projekten nach wie vor Nutzen aus ihrem AT:net Projekt ziehen.

Ein wesentliches Element der Bewertung der Projektergebnisse und eine mögliche Erklärung für die Nachhaltigkeit der (verwerteten) Projektergebnisse ist die Frage, ob es sich um Marktneuheiten oder Neuheiten für die Organisation handelte (bzw. voraussichtlich handeln wird). Die folgende Abbildung 39 zeigt, dass der überwiegende Anteil der Projekte bzw. befragten Organisationen inre Projektergebnisse als Marktneuheit einschätzt (69\% der abgeschlossenen und 65\% der laufenden Projekte). Einschränkend muss hinzugefügt werden, dass einige der befragten Organisationen in geographisch eher eingeschränkten, d.h. vor allem regionalen, Märkten aktiv sind. 


\section{Abbildung 39 Innovationsgrad der Projektergebnisse bei Markteintritt}

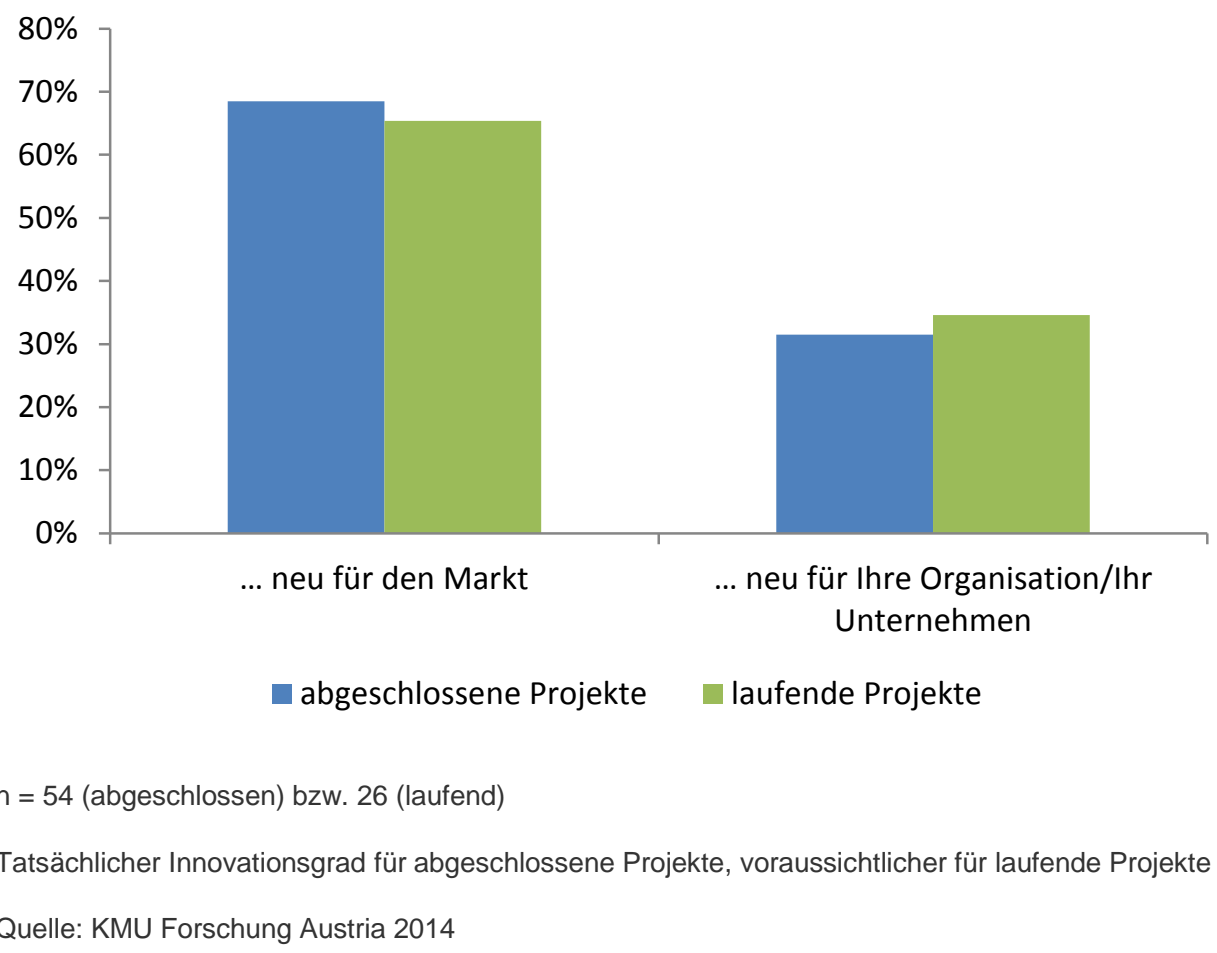

Aus Sicht der Fördernehmer ist die Frage nach der gesamten Kosten-NutzenRelation (der abgeschlossenen Projekte) ganz überwiegend positiv zu beantworten. Demnach sieht die Hälfte der Organisationen bereits jetzt, dass der Nutzen die Kosten des Projekts übersteigt und weitere $42 \%$ erwarten dies für die Zukunft. Dabei ist zu beachten, dass die entsprechende Fragestellung die Befragten explizit um eine Gesamtbewertung einschließlich nicht-monetärer Aspekte gebeten wurden. Im Zusammenhang mit der Diskussion der Zielerreichung (siehe Abbildung 36) und der Projektwirkungen (siehe Kapitel 5.4.2) lässt sich erkennen, dass eine überwiegende Mehrheit der Fördernehmer einen erheblichen Nutzen aus ihrem AT:net Projekt zu ziehen vermochten und dass dieser Nutzen eben nur zum Teil unmittelbare, messbare Folgen zeitigt. 


\section{Abbildung 40 Kosten-Nutzen-Relation der abgeschlossenen Projekte}

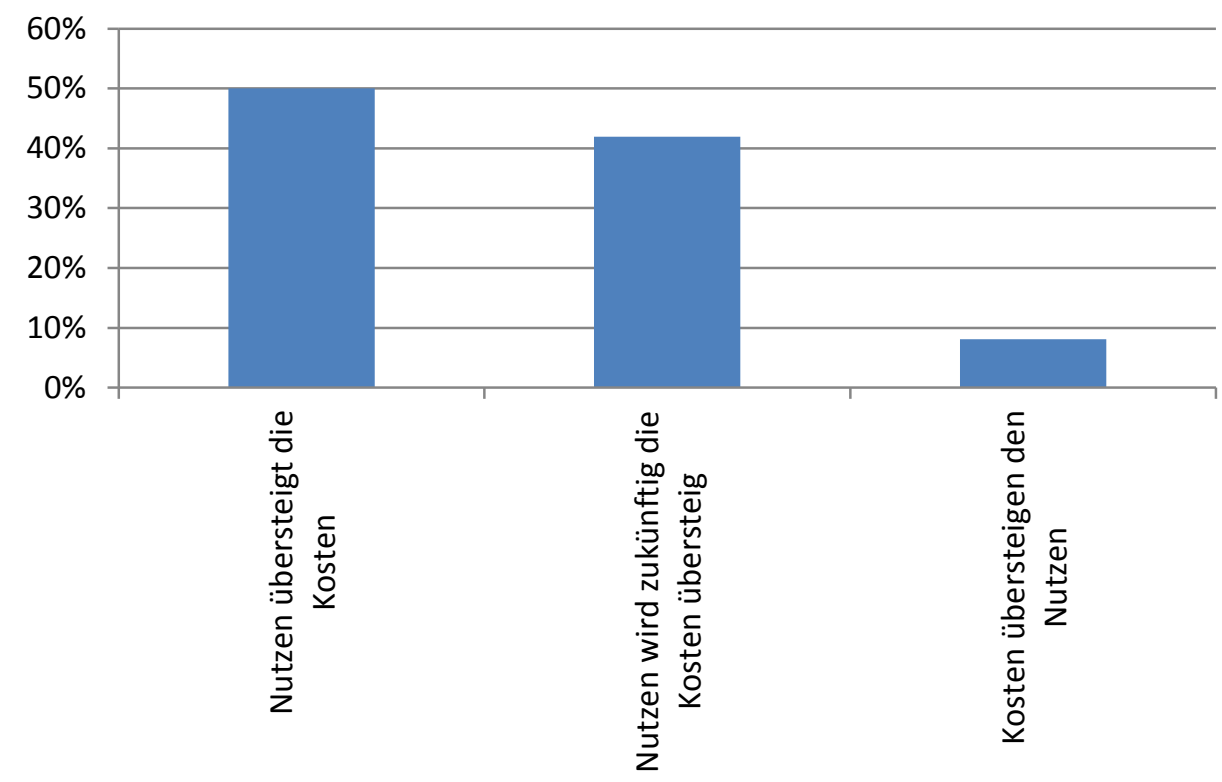

$N=62$

Quelle: KMU Forschung Austria 2014

\subsubsection{Wirkungen}

Obwohl 75\% der Befragten mit abgeschlossenen Projekten angeben, ihre wirtschaftlichen Projektziele erreicht zu haben (siehe Abbildung 36), haben nur $40 \%$ zusätzliche Umsätze (bzw. Drittmittel für Forschungseinrichtungen und Hochschulen) erzielt, die direkt dem Projekt zuzuordnen sind (siehe Abbildung 41). Die GröBe entsprechender Effekte ist deutlich heterogen: 20 Organisationen, die Umsatzwachstum aufgrund ihres AT:net Projekts verzeichnen konnten, gaben eine Zunahme zwischen 2 und $65 \%$ an. In erster Linie sind die Umsatzzuwächse größer je geringer der Jahresumsatz ist. 
Abbildung 41 Zusätzliche erzielte Umsätze/Drittmittel, die direkt dem Projekt zugeordnet werden können

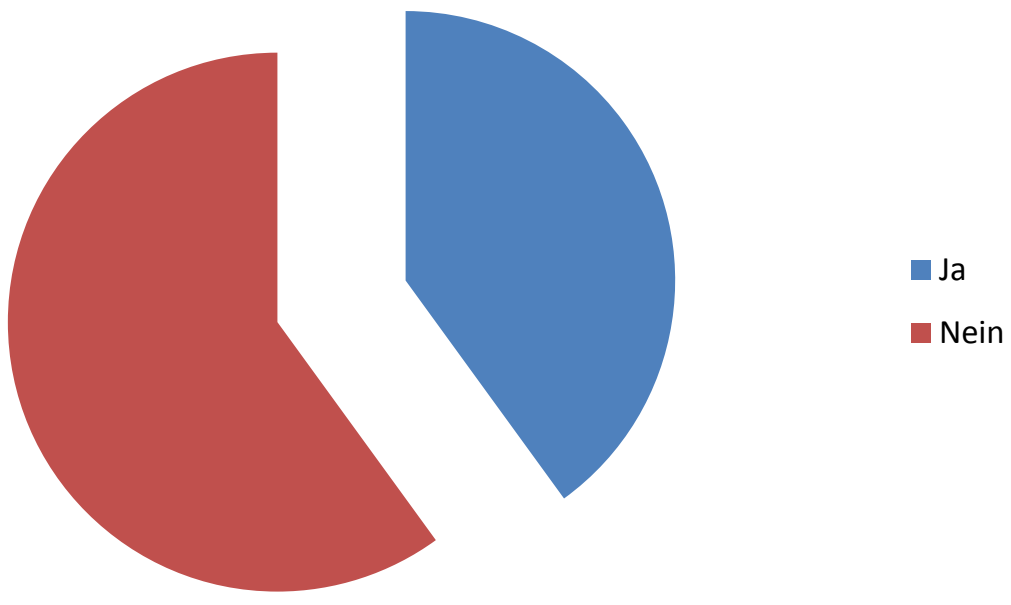

$\mathrm{n}=55$

Quelle: KMU Forschung Austria 2014

Deutlich größer ist dagegen der Anteil der Befragten, die eine positive Arbeitsplatzwirkung des Projekts auf die Organisation bestätigen: $34 \%$ gaben an, dass mithilfe des Projektes bzw. Projektergebnisses Arbeitsplätze gesichert werden konnten und weitere $18 \%$ konnten sogar zusätzliche Mitarbeiter einstellen. 


\section{Abbildung 42 Arbeitsplatzwirkungen die direkt dem Projekt zugeordnet werden} können, in Prozent aller befragten
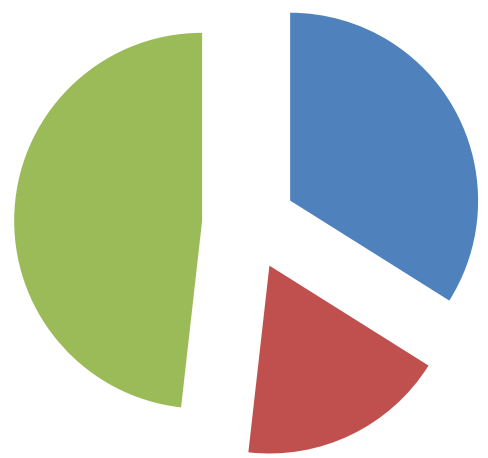

Ja, es wurden Arbeitsplätze gesichert.

a Ja, es wurden mehr MitarbeiterInnen eingestellt als freigesetzt.

Nein

$N=56$

Quelle: KMU Forschung Austria 2014

Eine Interpretation des relativ geringen Anteils der Organisationen mit positiven Umsatzwirkungen besteht auch angesichts der dominierenden Arbeitsplatzsicherung gegenüber der Neuschaffung zusätzlicher Arbeitsplätze sicherlich darin, dass innovative Produkte, Dienstleistungen usw. oftmals bereits bestehende ersetzen, also gegebenenfalls modernisierte Formen dieser darstellen. Während dies für eine Mehrheit der Fördernehmer gilt, sind unter den befragten Fördernehmern auch solche, deren Umsatz sich in Folge des Projekts deutlich verbessert hat (das Maximum liegt bei $65 \%$ des Jahresumsatzes). Allerdings sind unter den Befragten nur zwei Unternehmen, deren Umsatz sehr deutlich (d.h. 50\% oder mehr) gewachsen ist und jene mit hohen Umsatzzuwächsen sind eher kleine Unternehmen.

Relevanter als direkte Umsatz- oder Arbeitsplatzwirkungen ist die Erschließung neuer Märkte bzw. Marktsegmente, was auf mehr als 70\% der Befragten zutrifft (siehe Abbildung 43). Die geförderten Organisationen haben dabei vor allem nationale Märkte, gefolgt von internationalen und regionalen, erschließen können. 


\section{Abbildung 43 Erschließung neuer Märkte in Folge des geförderten Projekts}

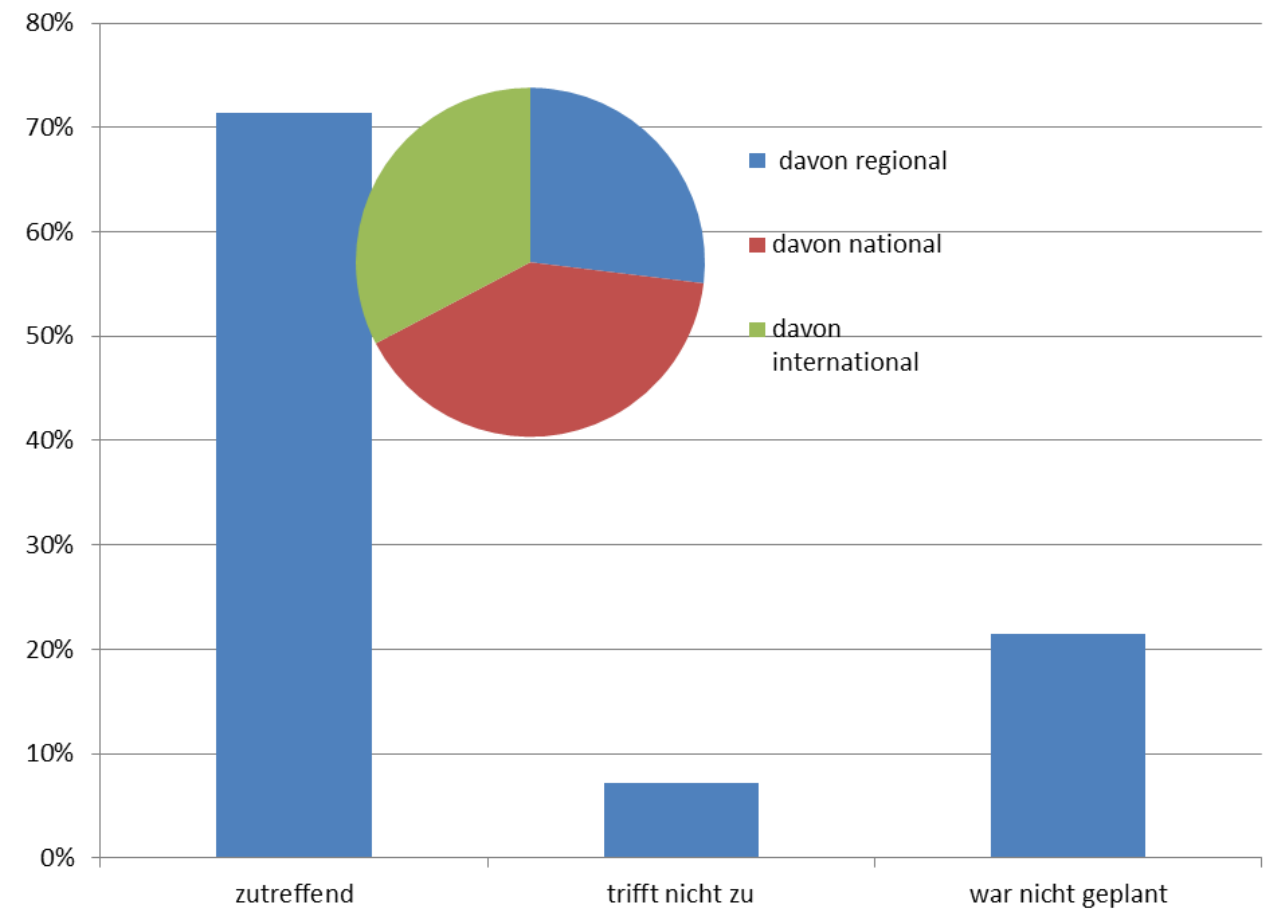

$\mathrm{n}=56$

Quelle: KMU Forschung Austria 2014

Darüber hinaus sind weitere Effekte aufgrund der AT:net Projekte erzielt worden (siehe Abbildung 44). Am häufigsten waren die Organisationen in der Lage, neue Kunden zu gewinnen (57\%), neue Kundensegmente zu erschließen (48\%) und die allgemeine Wettbewerbsfähigkeit zu stärken (47\%). Während diese Zahlen den Projekten positive Wirkungen auf Einzelorganisationsebene zusprechen, bzw. insgesamt eine positive Wirkung im Sinne der Programmziele, ist gleichzeitig auffällig, dass eine knappe Mehrheit nicht in der Lage war, einen so allgemeinen, eher „weichen" Effekt wie die gesteigerte Wettbewerbsfähigkeit zu erzielen. Die Unterschiede zwischen den abgeschlossenen und laufenden Projekten sind einerseits deutlich, etwa die deutlich höheren Anteile der geplanten Gewinnung neuer Kunden bzw. Erschließung neuer Kundensegment, sind aber andererseits eher als Folge der unterschiedlichen Projektstruktur (unter den laufenden Projekten sind relativ mehr Infrastrukturprojekte) zu betrachten als einer grundsätzlich Abweichung von Planung und Realität zuzurechnen. 


\section{Abbildung 44 Weitere Effekte als Folge des geförderten Projekts, in Prozent aller befragten Organisationen}

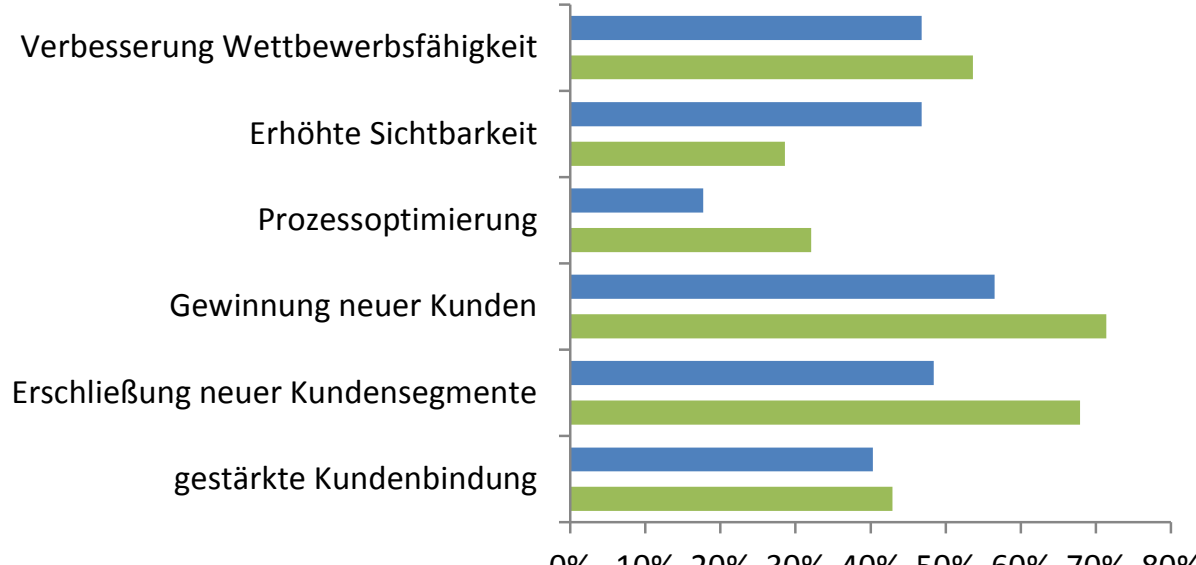

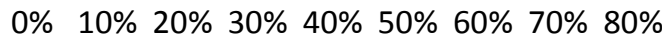

abgeschlossene Projekte — laufende Projekte

n $=56$ (abgeschlossen) bzw. 27 (laufend)

Quelle: KMU Forschung Austria 2014

Diejenigen Fördernehmer, die eine explizite Weiterführung eines Forschungsprojekts als Basis ihres AT:net Projekts angaben, bleiben hinsichtlich aller diskutierten Wirkungen innerhalb der Parameter. Eine besondere Hebelwirkung bei solchen Projekten ist daher nicht anzunehmen.

Im Sinne öffentlicher Innovationsförderung sind neben den Wirkungen auf Ebene der einzelnen Fördernehmer (und somit in Kumulation auf das gesamte Innovationssystem bzw. die Volkswirtschaft) auch jene Beiträge der einzelnen Projekte auf die Programmziele (siehe Abbildung 45). Abgesehen vom Globalziel der verstärkten Nutzung von Breitbandinternet, schätzen die meisten Befragten die Wirkung inres Projekt als schwach bis moderat ein, was allerdings wenig überrascht, da diese Programmziele aus Sicht einzelner Fördernehmer relativ abstrakt und stark aggregiert sind. Die insgesamt zweithöchste Bewertung des Beitrags lässt sich für die Frage gestiegenen Vertrauens in IKT-Systeme (und -Anwendungen) erkennen: $50 \%$ der befragten Fördernehmer erkennen hier einen (eher) signifikanten Beitrag ihres AT:net Projekts. Weitere $40 \%$ sehen einen solchen Beitrag im Bereich der Sicherheit von IKT-Systemen. In Anbetracht der Tatsache, dass diese Ziele von eher projektspezifischer Relevanz sind, können diese Ergebnisse als überraschend hoch angesehen werden. Das Gegenteil gilt für die Aussagen zur Wirksamkeit der Projekte im Bereich der erhöhten Teilhabe, für die zwar 36\% einen (eher) signifikanten Effekt erkennen, weitere 36\% aber überhaupt keinen. Dies ist um so kritischer, da der so genannte "digital divide“ einen hohen Stellenwert für das Programm AT:net hat. Die überwiegende Ablehnung von Projektwirkungen auf spezifische Nutzungsdimensionen von Breitbandinternet wie eGovernment, eLear- 
ning und eCommerce lässt sich wiederum mit der stark projektabhängigen Bearbeitung entsprechender Lösungsansätze erklären. Die hohe Ablehnungsquote (77\%) einer generellen Erhöhung der Nutzung von IT-basierten Dienstleistungen wirkt in diesem Zusammenhang überraschend, zumal Ausrichtung des Programms (d.h. Vermarktung von Anwendungen, die im IKT-Bereich eben ganz überwiegend Dienstleistungen sind) und die sonstigen Wirkungsdimensionen erwarten ließen, dass gerade in diesem Bereich viele Projekte einen signifikanten Beitrag zu leisten vermochten. Ob es sich bei diesem Ergebnis jedoch um einen Hinweis auf eine „verfehlte" Wirkung des Programms handelt, kann aus den Daten heraus nicht beantwortet werden. 
Abbildung 45 Beitrag der abgeschlossenen geförderten Projekte zu ProgrammzieIen

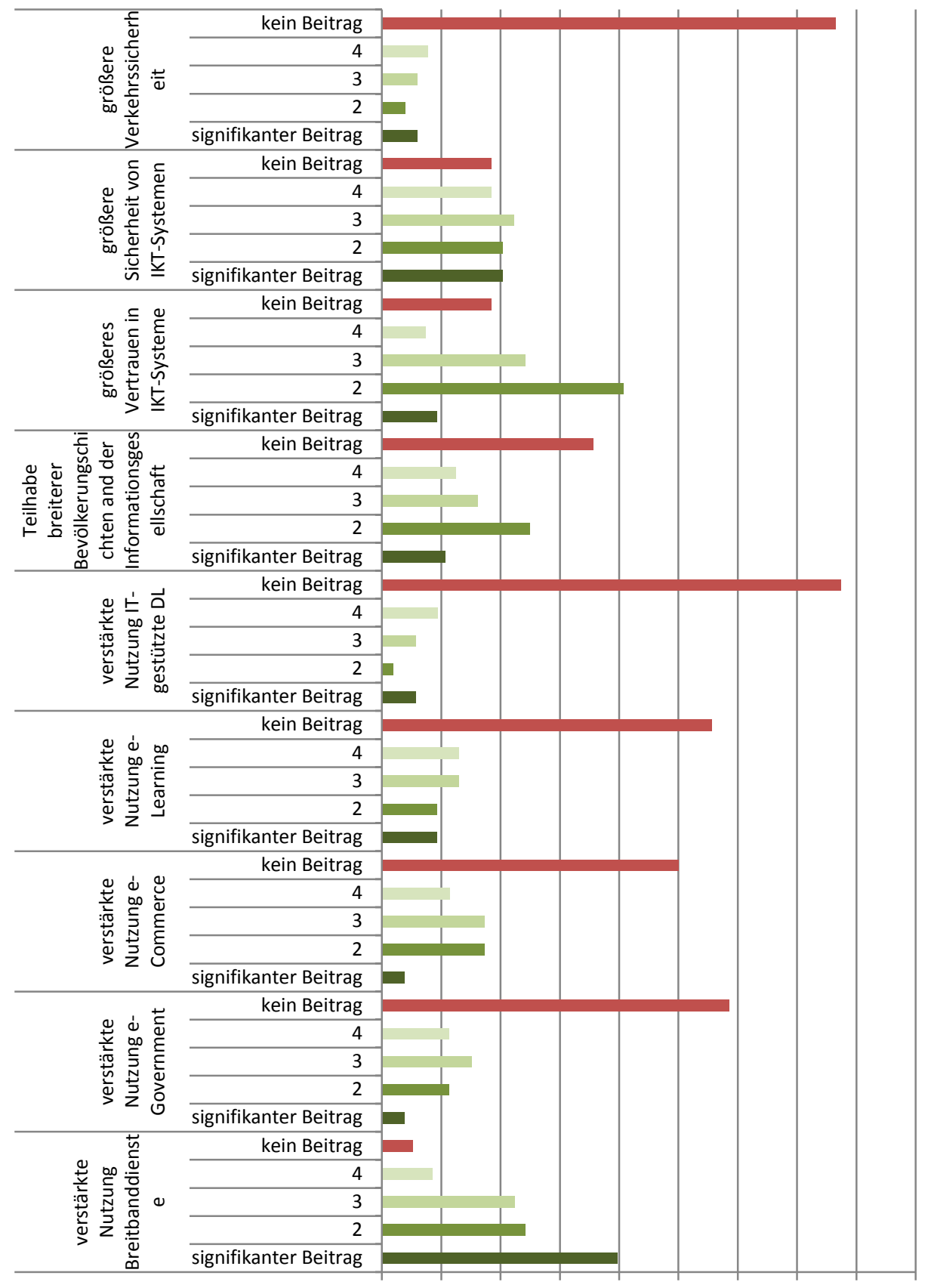

$\begin{array}{lllllllllllllll}0 \% & 10 \% & 20 \% & 30 \% & 40 \% & 50 \% & 60 \% & 70 \% & 80 \% & 90 \%\end{array}$

$\mathrm{n}=60$

Quelle: KMU Forschung Austria 2014

Insgesamt entsteht der Eindruck, dass der überwiegende Teil der Wirkungen auf der Eben der einzelnen Fördernehmer entsteht und (zumindest bis zum Zeitpunkt 
der Befragung) kaum Effekte auf die in den Programmdokumenten enthaltenen Makroziele erreicht wurde. Einerseits ist dies sicher dem Umstand geschuldet, dass letztere sehr abstrakt und ambitioniert sind (zumal im Zusammenhang mit einer eher niedrigschwelligen, finanziell eingeschränkten Förderung wie sie in AT:net möglich ist), kann andererseits aber zumindest als ein Anzeichen für eine Diskrepanz zwischen Projekten und Programm gewertet werden.

Die folgende Abbildung 46 fügt dieser Analyse eine weitere Ebene hinzu, indem die voraussichtlichen Wirkungen der noch laufenden Projekte erhoben und ausgewertet wurden. Insgesamt wird im Vergleich deutlich, dass der Anteil der Fördernehmer laufender Projekte, die einen signifikanten Beitrag zu den verschiedenen Programmzielen annehmen, leicht bis deutlich höher ist als unter den abgeschlossenen Projekten. Vor allem für das Globalziel einer verstärkten Nutzung von Breitbanddiensten sehen $67 \%$ einen signifikanten Beitrag ihres Projektes und weitere $22 \%$ einen eher signifikanten Beitrag. Eine Analyse, ob es sich dabei um eine realistische Einschätzung, übertriebenen Optimismus oder projektspezifische Bewertungen handelt, ist derzeit jedoch (noch) nicht möglich. Für letzteres sprechen allerdings die höheren Bewertungen der Signifikanz bezüglich konkreter Aspekte wie des Beitrags zu eGovernment (26\% mit signifikantem oder eher signifikantem Beitrag gegenüber $15 \%$ für die abgeschlossenen Projekte), eCommerce (35\% gegenüber $21 \%$ ) und eLearning (40\% gegenüber 19\%). Auch für den Themenkomplex der verbesserten Inklusion scheinen die laufenden Projekte einen höheren Beitrag zu erwarten als die abgeschlossenen zu leisten vermochten (21\% mit signifikantem Beitrag im Unterschied zu 11\%). 


\section{Abbildung 46 Beitrag der laufenden geförderten Projekte zu Programmzielen}

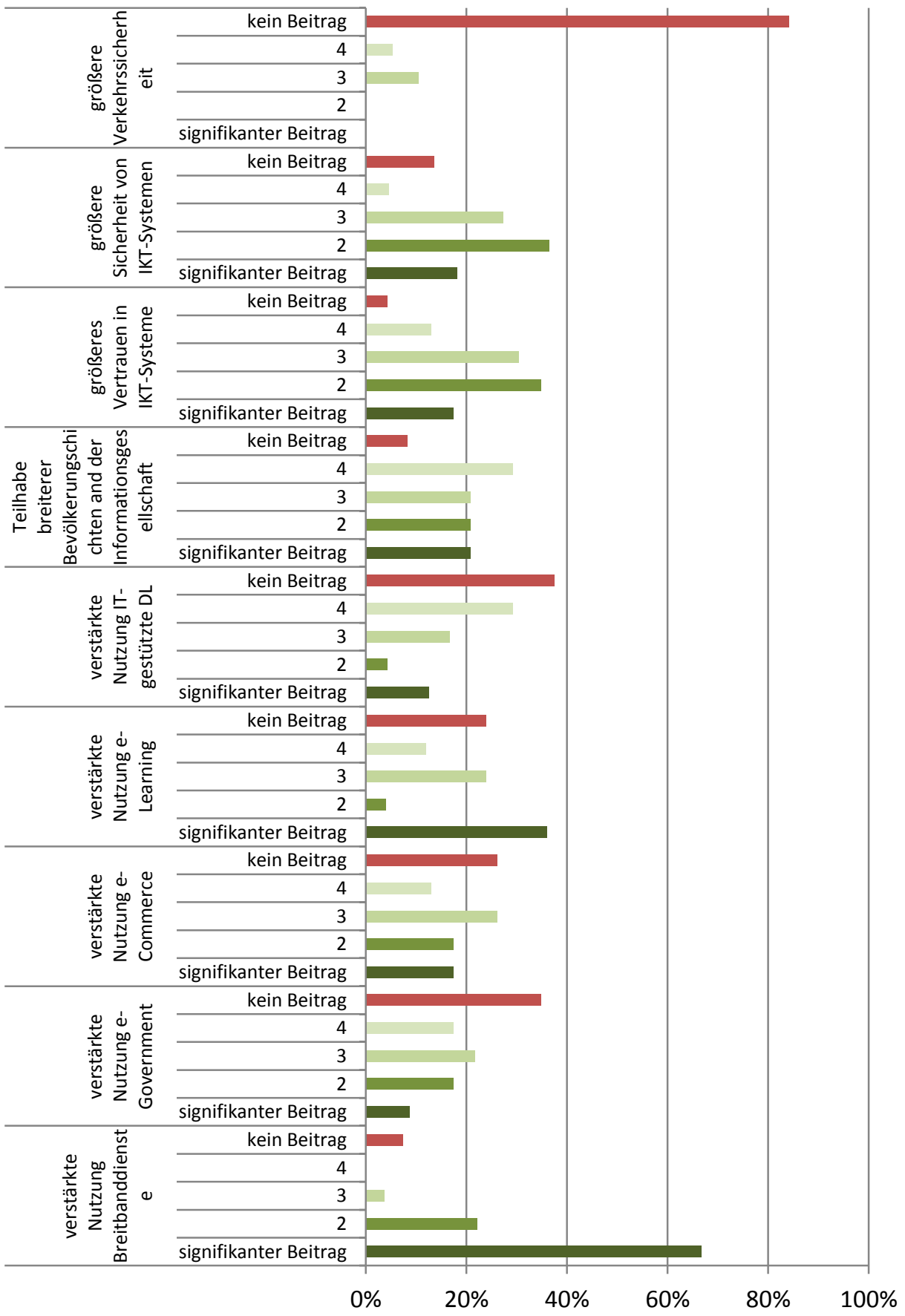

$\mathrm{n}=27$

Quelle: KMU Forschung Austria 2014 


\section{Schlussfolgerungen und Handlungsemp- fehlungen}

\subsection{Schlussfolgerungen}

Die strategischen Programmziele des Förderprogramms AT:net beinhalten vor allem „die Unterstützung der Einführung von qualitativen Diensten und Anwendung sowie der innovativen Verwertung von Forschungsergebnissen. [...] Breitbandausbau und die Förderung von [...] Zugängen sowie [...] Verringerung der digitalen Kluft [...]. Auf Basis der Befragung der Fördernehmer kann eine zumindest teilweise Erreichung dieser Ziele angenommen werden.

Die Mehrheit der geförderten Projekte hat zur Vermarktung einer neuen Dienstleistung, eines neuen Produkts etc. geführt. Die Wirkungen sind vor allem für die beteiligten Unternehmen als nachhaltig zu betrachten, da mehr als $90 \%$ der befragten Fördernehmer die entsprechenden Entwicklungen nach wie vor in ihrem Angebotsbzw. Produktportfolio haben. Der Selbsteinschätzung der befragten Organisationen zufolge handelt sich dabei in den allermeisten Fällen um Marktneuheiten, auch wenn die Referenz (d.h. regionale, nationale oder globale Märkte) nicht für alle Fördernehmer gleich ist. Für etwa zwei Drittel der Projekte kann ein Beitrag zur unmittelbaren Nachfragesteigerung angenommen werden (der sich auch in der entsprechenden Gewinnung neuer Kunden bzw. Kundensegmente widerspiegelt), allerdings sind diese Wirkungen nur schwer quantifizierbar und demzufolge ihr Effekt auf die Nachfrage nicht messbar. Hinzu kommt, dass eine gesteigerte Nachfrage auch nur dann eine Auswirkung auf den Breitbandausbau haben kann, wenn eine kritische Masse erreicht wird und der Ausbau von Breitbandverbindungen zur Befriedigung dieser gesteigerten Nachfrage überhaupt notwendig wäre. Insbesondere die Frage nach der kritischen Masse lässt sich auf Basis der existierenden und erhobenen Daten nicht beantworten. Für die geförderten Organisationen und insbesondere Unternehmen ist durch die Befragung der Fördernehmer deutlich geworden, dass AT:net nichtsdestotrotz von teilweise entscheidender Bedeutung für die erfolgreiche Markteinführung von innovativen Dienstleistungen und Produkten ist.

Trotz einer überwiegend positiven Kosten-Nutzen-Relation sind tatsächliche Zuwächse an Umsatz (bzw. Drittmittelumsatz für Forschungseinrichtungen und Hochschulen) und Beschäftigung ebenfalls nur für eine Minderheit Folge ihrer geförderten Projekte. Deutlich stärker ist die Wirkung des Programms hinsichtlich der Sicherung von Umsätzen und Arbeitsplätzen. Unter Berücksichtigung der Tatsache, dass Innovationen oftmals bereits existierende Produkte bzw. Dienstleistungen ersetzen, ist dieses Ergebnis, gemeinsam mit den sonstigen ökonomisch relevanten erzielten Wirkungen wie die Erschließung neuer Märkte, Kundensegmente etc., 
dennoch als Erfolg des Programms zu bewerten. Diese Effekte sind überwiegend als sehr heterogen zu betrachten. Sieht man sich etwa die erzielten Umsatzzuwächse - wo vorhanden - an, wird deutlich, dass diese von minimal ( $2 \%$ des Jahresumsatzes) bis deutlich (65\% des Umsatzes) reichen.

Ein direkter oder gar kausaler Zusammenhang mit der Entwicklung des Breitbandausbaus bzw. der entsprechenden Zugänge kann nicht nachgewiesen werden, abgesehen von den reinen Infrastrukturprojekten aus der zweiten Programmphase. Die Frage nach einer verringerten digitalen Kluft lässt sich ebenfalls nicht unmittelbar aus den geförderten Projekten heraus beantworten, ein direkter Beitrag muss jedoch als wenig wahrscheinlich gelten. Davon abgesehen ist zu beachten, dass die strategischen Ziele des Programms im Bereich Breitbandausbau, Förderung von Zugängen und Verringerung der digitalen Kluft überwiegend als kaum direkt erreichbar zu kennzeichnen sind. Innovative Breitbandzugänge im Sinne von etwa next generation access Technologien und die generelle Steigerung der am Markt verfügbaren Bandbreiten sind teilweise ohnehin bereits auf Basis der existierenden Infrastruktur zumindest in Ballungsgebieten möglich. Die Nachfrage danach ist eher über den Preis bzw. den Bedarf geregelt und letzterer ist mithilfe eines Förderprogramms wie AT:net kaum spürbar in der Gesamtbevölkerung zu steigern. Aus derzeitiger Sicht könnten etwa Streaming- oder andere Unterhaltungsdienste die Nachfrage spürbar steigen lassen, allerdings ist zu beachten, dass selbst für einen als disruptive Innovation (wobei die Innovation im Geschäftsmodell begründet liegt und nicht in der Technologie) bezeichneten Dienst wie das USamerikanische Netflix Bandbreiten von $10 \mathrm{Mb} / \mathrm{s}$ und weniger ausreichen ${ }^{5}$.

Hinsichtlich der Verwertung von Forschungsergebnissen ist der Befund weniger eindeutig. AT:net fördert aufgrund seiner Ausgestaltung und Zielgruppe eine heterogene Gruppe an Organisationen. Auf Basis der Befragung und der Analyse der sonstigen Beteiligungen an FFG Programmen ist davon auszugehen, dass nur ein Teil der Fördernehmer überhaupt eigene Forschungs- und Entwicklungsprojekte betreibt, die mithilfe von AT:net Projekten in die Vermarktung übergeleitet werden konnten. Sowohl die Projekte selbst als auch die Angaben der befragten Fördernehmer bestätigen die relativ geringe Bedeutung von AT:net als direkte Weiterführung von Forschungsaktivitäten. Weiterhin wurde deutlich, dass sich jene befragten Organisationen, die mithilfe explizit vorangegangene Forschungs- und Entwicklungsaktivitäten kommerzialisiert haben, hinsichtlich der mithilfe von AT:net erzielten Wirkungen kaum von den anderen Fördernehmern bzw. Projekten unterscheiden. Da sich unter den AT:net Fördernehmern jedoch auch solche Organisation, d.h. auch Unternehmen, die eine Vielzahl von Forschungsprojekten in anderen FFG Programmen durchgeführt haben, befinden, kann eine Relevanz von AT:net

\footnotetext{
${ }^{5}$ Kenny/Broughton, 2013, Domestic demand for bandwidth, An approach to forecasting requirements
} for the period 2013-2023. 
für forschende Organisationen und damit die Vermarktung von Forschungsaktivitäten angenommen werden. Die bewusst breite Aufstellung von AT:net als Wirtschaftsförderungsprogramm (und eben nicht als reines innovationspolitisches Instrument) mit Hinblick auf die potenziellen Fördernehmer kann damit als erfolgreich gekennzeichnet werden. Innovative, vermarktungsreife (d.h. erfolgversprechende) Ideen entstammen vor allem im IKT Bereich oft eben nicht klassischen Forschungs- und Entwicklungsprojekten.

Die operativen Zielsetzungen des Programms sind überwiegend erreicht worden. Allerdings lässt sich nicht quantifizieren, welchen Beitrag die AT:net Projekte zu leisten vermochten. Geht man etwa von der Selbsteinschätzung der befragten Fördernehmer aus, ist zumindest die Verringerung der digitalen Kluft kaum auf das Programm zurückzuführen. Überhaupt zeigen sich in den entsprechenden Daten der Statistik Austria einerseits Sättigungstendenzen, d.h. die erreichten Ergebnisse sind Resultat eines „natürlichen“ Wachstums und inzwischen im Bereich des erreichbaren Maximums, und andererseits zeigt sich vor allem beim Thema digitale Kluft, dass es sich möglicherweise um ein zumindest nicht mithilfe von Forschungs- oder Innovationsförderung behebbares Problem handelt.

Zusammengefasst lässt sich feststellen, dass AT:net für die beteiligten Fördernehmer und ihrer wirtschaftliche Leistungs- und Wettbewerbsfähigkeit von großer Bedeutung ist. AT:net weist relativ geringe Mitnahmeeffekte (d.h. Projekte, die auch ohne Förderung unverändert durchgeführt worden wären) auf und der überwiegende Teil der Projekte hätte ohne die Förderung in deutlich verringertem Ausmaß oder mit deutlicher zeitlicher Verzögerung durchgeführt werden müssen. Die intendierten Wirkungen auf Ebene einzelner Organisationen können als erreicht gelten, sind jedoch deutlich heterogen bezüglich ihrer Ausmaße.

Das Programm vermag es, aufgrund seiner Niedrigschwelligkeit und relativ einfachen Administration, auch solche Organisationen anzuziehen, die nicht zu den üblichen Fördernehmern der Forschungs- und Innovationspolitik zählen. Dieser Umstand kann trotz fehlender Quantifizierbarkeit vieler Effekte nicht gering geschätzt werden. Der Erfolg der grundsätzlichen thematischen Offenheit des Programms kann auf Basis der Analysen weiterer FFG-Förderungen der AT:net Fördernehmer bestätigt werden, da nur ein geringer Anteil auf IKT-spezifische Förderprogramme entfällt (thematisch offene Programme und Programme aus dem Themenbereich Mobilität und Verkehr sind deutlich relevanter).

Kritische Masse bezüglich der intendierten Nachfragestimulation auf gesamtgesellschaftlicher Ebene ist insgesamt schwer nachweisbar, da nur punktuell, d.h. in vereinzelten Projekten, Effekte entstanden sind, die Anlass zur Vermutung geben, dass AT:net hier ursächlich an der Entwicklung der Makroindikatoren zur Breitbandnutzung und zum Breitbandausbau beitragen konnte. Vor dem Hintergrund der sich verändernden Problematik, d.h. Sättigungstendenzen bei den Breitbandanschlüssen und im internationalen Vergleich fehlender Verbreitung von next ge- 
neration access Technologien sowie geringerer Bandbreiten, ist es ohnehin fraglich, ob AT:net hier mit den „richtigen“ Zieldimensionen ausgestattet ist bzW. es für die Zukunft nicht einer umfassenden Neuausrichtung bedarf. Neben dem grundsätzlichen potenziellen Wirkungskonflikt von (durch Förderung) erreichbaren Effekten und operativen sowie strategischen Zielsetzungen, ist vor allem die Diskrepanz zwischen Projektgröße (bedingt durch die Verwendung von de minimis) und der notwendigen kritischen Masse (auf Basis der verwendeten Zielsetzungen) zu beobachten. Letztere wäre zweifelsohne notwendig, sollte AT:net einen sichtbaren Beitrag zu den eher gesamtgesellschaftlichen Zieldimensionen leisten, während ersteres einen nicht unerheblichen Anteil an sowohl der Attraktivität als auch den erzielten Erfolgen (für die einzelnen Fördernehmer) trägt.

\subsection{AT:net im internationalen Vergleich}

AT:net hat, wie beschrieben, zwei zentrale Elemente, die Basis für einen Vergleich mit Förderinstrumenten in anderen Staaten sein können: die Zielsetzung der zunehmenden Breitbandnutzung und des Infrastrukturausbaus sowie die Unterstützung von innovativen IKT-Anwendungen. Im internationalen Vergleich wird deutlich, dass Programme zur Innovationsförderung auch im Bereich Breitbandinternet auch bei Nutzung einer ähnlichen Interventionslogik (Förderung von Nachfrage als Hebel) in erster Linie nicht-rückzahlbare Zuschüsse in forschungsintensiven, vorwettbewerblichen Einzel- oder Kooperationsprojekten nutzen ${ }^{6}$. Diese Programme unterstützen im Vergleich zu AT:net eher größere Projekte, allerdings nicht über die Entwicklung eines Prototypen hinaus. Eine aktive Förderung der Marktüberleitung ist in solchen Programmen in aller Regel weder möglich noch vorgesehen. Vor diesem Hintergrund ist es von Interesse, dass die Europäische Kommission mit dem Forschungsrahmenprogramm Horizon 2020 einen anderen Weg geht, nämlich insgesamt weg von der reinen Projektförderung hin zur Förderung von Ideen über die gesamte Innovationskette, an deren Ende die Vermittlung von Risiko- oder Beteiligungskapital durch die Europäische Investitionsbank EIB bzw. private Organisationen stehen soll. In diesem Verständnis nimmt AT:net eine förderungspolitische Innovation nicht nur vorweg, sondern bietet mit der Nutzung von de minimis eine Alternative zum in Europa mehrheitlich kaum vorhandenen staatlichem und privatem Risikokapital als Mittel zur Vermarktungsunterstützung. Mangels empirischer Nachweise ist derzeit nicht zu klären, welchen Ideen die Europäische Kommission über die erneuerte systemische Interventionslogik zu welchem Erfolg verhelfen wird/kann. Für eine eventuelle zukünftige Evaluierungen von AT:net, im Falle einer Weiterführung, könnte ein diesbezüglicher Vergleich dennoch von Interesse sein.

\footnotetext{
${ }^{6}$ Siehe etwa das finnische GIGA Converting Networks.
} 
Obwohl nachfrageorientierte Politikinstrumente zumindest für die Innovationspolitik seit längerer Zeit diskutiert werden (etwa öffentliche Beschaffung als indirektes Förderinstrument), sind sie in der Realität eher selten anzutreffen. AT:net mit seiner Interventionslogik der Nachfrageunterstützung als indirekte Unterstützung des Breitbandausbaus scheint ohne Pendant in anderen Staaten zu sein. Breitbandausbau wird in erster Linie entweder über reine Infrastrukturmaßnahmen gefördert (etwa wie in Großbritannien über staatliche Fonds, die Infrastrukturausbau in umgekehrten Versteigerungen an private Unternehmen vergeben) oder über die Unterstützung von Nutzung/Kauf von IKT-Equipment durch Unternehmen (vor allem $\mathrm{KMU})$.

\subsection{Handlungsempfehlungen}

Angesichts der erzielten Wirkungen zumindest auf Ebene der einzelnen Fördernehmer kann AT:net insgesamt als Erfolg gewertet werden. Der kaum zu erbringende Nachweis des Beitrags der einzelnen Projekte zu den eher gesamtgesellschaftlichen Indikatoren und Zielen mindert diesen nur teilweise. Die Nutzung von de minimis und die damit einhergehende Niedrigschwelligkeit des Programms sind zentrale Erfolgsfaktoren, vor allem vor dem Hintergrund der Unterstützung von Unternehmen außerhalb der forschungsintensiven, klassischerweise mithilfe von Förderprogrammen erreichten Branchen. Daher spricht alles dafür, AT:net im Wesentlichen in seiner jetzigen Form weiterzuführen, um wie bisher die gesamte Bandbreite möglicher Anwendungen und Fördernehmer zu erreichen, auch vor dem Hintergrund, dass Breitbandausbau und die Nachfrage danach sowie die Adressierung letzterer als Instrument der Unterstützung auch zukünftig von wirtschaftspolitischer Relevanz sein werden.

Empfehlung 1: AT:net sollte als breitenwirksames Instrument der Vermarktungsunterstützung innovativer IKT-Anwendungen erhalten bzw. neu aufgelegt werden.

Aufgrund der kaum nachweisbaren Wirkung der einzelnen Projekte auf die gesamtgesellschaftlichen Indikatoren wie die Verbreitung und Verfügbarkeit von Breitbandinternet und vor dem Hintergrund der Breitbandstrategie 2020 des BMVIT sollte AT:net zwar auch künftig mit solchen Indikatoren verknüpft sein, jedoch sollten diese nicht länger als operative Ziele dienen.

Empfehlung 2: Indikatoren des Breitbandausbaus und der Breitbandnutzung sollten zukünftig nicht länger als operative Ziele des Programms genutzt werden, sondern im Sinne einer Verknüpfung mit der Breitbandstrategie 2020 nur mehr als strategische Zielsetzungen.

Mit der Breitbandstrategie 2020 (und der Einrichtung eines Breitbandbüros) steht eine österreichische Strategie zur Verfügung, die als Leitfaden und konzeptioneller Überbau für AT:net genutzt werden könnte. Die darin dokumentierten Ziele und 
Themen (z.B. Interoperabilität und Netzneutralität) finden sich entweder bereits (in Ansätzen) in AT:net wieder oder sind zumindest kompatibel mit der existierenden Interventionslogik und der Ausrichtung des Programms. Eine Neuauflage von AT:net müsste explizit mit diesen Entwicklungen verknüpft werden und die Zielsetzungen sollten angepasst werden, um im Einklang mit der Breibandinitiative des BMVIT und europäischen IKT-Agenden zu stehen.

Empfehlung 3: AT:net sollte in einer Neuauflage explizit und direkt mit der Breitbandstrategie 2020 und der Digital Agenda der EU Kommission verknüpft sein, d.h. die entsprechenden Ziele und Themen sollten zentrale Elemente der strategischen (und operativen) Ziele des Programms sein.

Vor dem Hintergrund der nachweisbaren Wirkungen müssten vor allem die operativen Ziele im Sinne einer expliziten Funktion von AT:net innerhalb der Breitbandstrategie 2020 angepasst werden. Die ursprüngliche Interventionslogik bliebe davon unberührt, nur dass die Zielindikatoren von Breitbandverfügbarkeit und -nutzung stärker in Richtung Kommerzialisierung von innovativen IKT- (bzW. Breitband-) Anwendungen (z.B. Unternehmenswachstum durch innovative Produkte und Dienstleistungen, Wettbewerbsfähigkeit, Export- und Marktanteile etc.) verändert werden müssten. Die Darstellung der entsprechenden Potenziale der Projekte müsste bereits in den Förderungsanträgen erfolgen und stärker als bisher zu einem Evaluationskriterium werden. Unweigerlich würde dies den Aufwand für die potenziellen Fördernehmer und die Evaluierung erhöhen, d.h. eine sorgfältige Abstimmung von zusätzlichen zu leistenden Nachweisen durch die potenziellen Fördernehmer und dem Erhalt der für AT:net erfolgsrelevanten Niedrigschwelligkeit ist unabdingbar. Die bereits vorgenommenen Veränderungen in der Programmbewerbung sowie den Projektanträgen gehen ohnedies bereits in diese Richtung.

Empfehlung 4: Die operativen Zielsetzungen und -indikatoren von AT:net sollten stärker die tatsächlichen Wirkungen sowie ganz allgemein nachweisbare Effekte berücksichtigen. Da diese in erster Linie in einer gestärkten Leistungs- und Wettbewerbsfähigkeit vor allem der Unternehmen bestehen, sollten diese entsprechend angepasst werden: Indikatoren des Unternehmenswachstums (bzw. der Sicherung und des Bestands der Unternehmen) wie Umsatzentwicklung, Exportentwicklung, Marktanteile, Marktzugänge, Produktivitätsentwicklung, Nutzer- bzw. Kundenzahlen. Ergänzend sollten Indikatoren im Bereich Innovation/Technologie genutzt werden, wie etwa Anregung weiterer Entwicklungen, Einstieg in neue Technologie/Anwendungsfelder, technologischer Sprung. 
Empfehlung 5: AT:net als Unterstützungsleistung für die Vermarktung von innovativen auf Breitbandnutzung aufbauenden IKT-Anwendungen sollte bereits in der Phase der Antragstellung bzw. Antragsevaluation entsprechende Kriterien beinhalten. Antragsteller sollten dazu Meilensteine und Vermarktungsstrategien erarbeiten, deren Relevanz, Erreichbarkeit, Vollständigkeit sowie Flexibilität (im Sinne einer Risikominimierungsstrategie) zentrale Bewertungskriterien darstellen.

Empfehlung 6: Um den zusätzlichen Aufwand gegenüber der Niedrigschwelligkeit von AT:net zu minimieren, sollte die Möglichkeit eines Angebots einer geförderten Beratungsleistung (durch die FFG oder extern) geprüft werden (eventuell auch erst sobald ein positiver Förderbescheid ergangen ist).

IKT sind per se ein dynamisches Themenfeld und die entsprechenden Anwendungen müssen sich in einem ebenso dynamischen Markt- und Wettbewerbsumfeld behaupten können. Die Tatsache, dass AT:net bereits eine große Bandbreite von Organisationen und Innovationen unterstützt, heißt nicht, dass die Gruppe potenziell interessanter Fördernehmer und IKT-Anwendungen nicht noch deutlich größer ist. Es ist nicht klar, inwieweit AT:net als geeignetes Förderinstrument umfassend wahrgenommen bzw. angenommen wird und ob die derzeitigen Anstrengungen im Bereich der Programmbewerbung ausreichend sind, um alle Entwickler passender Lösungen zu adressieren. Mit der Verschiebung des Fokus der Bewerbung des Programms etwa auf die österreichische Start-up Szene ist allerdings bereits ein Anfang gemacht, der nicht nur neue Fördernehmergruppen mit AT:net bzw. der Idee einer Wirtschafts- oder Innovationsförderung in Kontakt bringt, sondern auch den Innovationsbezug sowie die potenziellen Effekte des Programms deutlich verstärken könnte, ohne an der erfolgreichen Interventionslogik Veränderungen vornehmen zu müssen und damit die Niedrigschwelligkeit von AT:net zu gefährden (und auf andere abschreckend zu wirken).

Empfehlung 7: Die Bewerbung von AT:net hat sich nicht nur als wichtiges und erfolgreiches Instrument erwiesen, sondern kann auch als indirektes Steuerungsmittel zur Erhöhung der Wirksamkeit des Programms genutzt werden. Daher sollte das Programm in Abstimmung mit den strategischen und operativen Zielsetzungen zukünftig stärker in der österreichischen Start-up und (Software-) Entwickler-Szene auf den entsprechenden Veranstaltungen bzw. in den relevanten Medien beworben werden.

AT:net ist per se als - im Rahmen von Breitbandrelevanten IKT-Anwendungen und entsprechender Dienstleistungen - als thematisch offenes Förderprogramm entwickelt worden. Gleichzeitig existieren jedoch mehrere Themenfelder, die in der Programmdokumentation und -bewerbung genannt werden. Aufgrund der limitierten Beiträge der einzelnen Projekte zu entsprechenden Zielen, wie etwa der verstärkten Nutzung von eCommerce oder eGovernment, erscheint es als wenig zielfüh- 
rend, diese thematische Einteilung auf die eigentlichen Projekte anzuwenden. Dies ist auch und gerade vor dem Hintergrund einer Interventionslogik, die dem Faktor Nachfrage die Hauptrolle zuweist, und daher eine Vorgabe von top-down Themenfeldern eigentlich vermeidet, zumindest widersprüchlich. Die Frage, inwieweit dadurch potenzielle Fördernehmer von einer Einreichung abgeschreckt werden, ist auf Basis der verfügbaren und erhobenen Informationen nicht zu beantworten. Um das Programm AT:net jedoch hinsichtlich der Wahrnehmung und Nutzung weitergehend auf seinen eigentlichen Kern zu fokussieren, sollten thematische Einschränkungen vollkommen beseitigt werden und Themenfelder in der Kommunikation und Bewerbung nur mehr zur Beschreibung möglicher Anwendungsbereiche dienen. Diese müssten dann allerdings einer regelmäßigen Überprüfung und Anpassung unterworfen werden. Des Weiteren wäre ebenso regelmäßig zu prüfen, ob durch entsprechende Veränderungen in der Außendarstellung eine Steuerungswirkung entsteht, die zu einer vermehrten Einreichung von Projekten in bestimmten Themenbereichen führt. Je nachdem könnte eine solche variierende Kommunikation, wie die vorgeschlagene und bereits begonnene Neuausrichtung der Bewerbungsstrategie des Programms an sich, als weiches Steuerungsinstrument ausgebaut werden.

Empfehlung 8: Thematische Vorgaben, abseits von der generellen Einschränkung auf breitbandbasierten IKT-Anwendungen und Dienstleistungen, widersprechen der marktgetriebenen Interventionslogik des Programms AT:net. Themen- und Anwendungsfelder sollten in einer Neuauflage nur mehr als inhaltiche Orientierung dienen und entsprechend kommuniziert werden, müssten dann aber vor allem umfassend sein, um keine abschreckende Wirkung auf potenzielle Fördernehmer $z u$ entwickeln. Dabei ist zu prüfen, ob etwa aufgrund neuer technologischer Entwicklungen regelmäßige Anpassungen in der Auflistung und Beschreibung dieser Felder notwendig und sinnvoll sein könnten. Weiterhin sollte die Möglichkeit geprüft werden, ob durch eine regelmäßig adaptierte Außenkommunikation Steuerungseffekte hinsichtlich der eingereichten Projektanträge und ihrer Themen möglich bzw. sinnvoll sind. 
KMU Forschung Austria

Austrian Institute for SME Research 